\title{
An Unbounded Generalization of the Tomita-Takesaki Theory II
}

\author{
By
}

\author{
Atsushi INOUE*
}

\begin{abstract}
An unbounded generalization of the fundamental concepts of the Tomita-Takesaki theory such as modular automorphism groups and Radon-Nikodym derivatives is considered.

\section{\&. Introduction}

In this paper we continue our study of an unbounded generalization of the Tomita-Takesaki theory begun in a previous paper [14].

The Tomita-Takesaki theory shows that the vector state $\omega_{\xi_{0}}$ defined by a cyclic and separating vector $\xi_{0}$ for a von Neumann algebra satisfies the $\mathrm{KMS}$-condition with respect to the modular automorphism group $\left\{\sigma_{t}^{\xi_{0}}\right\}$. To extend these results to unbounded operator algebras, we define the notions of modular vectors, standard vectors and standard systems for a closed $O_{p}^{*}$-algebra $(\mathscr{M}, \mathscr{D})$. Using the unbounded Tomita-Takesaki theory developed in a previous paper [14], we show that if $\xi_{0}$ is a modular vector for $(\mathscr{M}, \mathscr{D})$ then a one-parameter group $\left\{\sigma_{t}^{\xi_{0}}\right\}$ of $*$-automorphisms of an unbounded bicommutant $\left(\mathscr{M} / \mathscr{D}_{\xi_{0}}\right)_{w c}^{\prime \prime}$ of the $O_{p}^{*}$-algebra $\mathscr{M} / \mathscr{D}_{\xi_{0}}$ on a dense subspace $\mathscr{D}_{\xi_{0}}$ of $\mathscr{D}$ is defined, and the vector state $\omega_{\xi_{0}}$ on $\left(\mathscr{M} / \mathscr{D}_{\xi_{0}}\right){ }_{w c}^{\prime \prime}$ satisfies the KMScondition with respect to $\left\{\sigma_{t}^{\xi_{0}}\right\}$.

We next apply the unitary Radon-Nikodym cocycle introduced by Connes [3] to the unbounded case. Let $(\mathscr{M}, \mathscr{D})$ be a closed $O_{p}^{*-}$ algebra and a pair $\left(\xi_{1}, \xi_{2}\right)$ of vectors in $\mathscr{D}$ be strongly cyclic for $\mathscr{M}$ and separating for the usual commutant $\mathscr{M}^{\prime \prime} \equiv\left(\mathscr{M}_{w}^{\prime}\right)^{\prime}$ of the weak commutant $\mathscr{M}_{w}^{\prime}$ of $\mathscr{M}$. Connes showed that the modular automorphism
\end{abstract}

Communicated by H. Araki, February 27, 1987.

* Department of Mathematics, Fukuoka University, Fukuoka 814-01, Japan. 
groups $\left\{\sigma_{t}^{\xi_{1}}\right\}$ and $\left\{\sigma_{t}^{\xi_{2}^{2}}\right\}$ of the von Neumann algebra $\mathscr{M}^{\prime \prime}$ satisfy the relation: $\sigma_{t}^{\xi_{1}}(A)=\left(D \omega_{\xi_{1}}^{\prime \prime}: D \omega_{\xi_{2}}^{\prime \prime}\right)_{t} \sigma_{t}^{\xi_{2}}(A) \quad\left(D \omega_{\xi_{1}}^{\prime \prime}: D \omega_{\xi_{2}}^{\prime \prime}\right)_{t}^{*}$ for all $t \in \mathbb{R}$ and $A \in \mathscr{M}^{\prime \prime}$, where $\left(D \omega_{\xi_{1}}^{\prime \prime}: D \omega_{\xi_{2}}^{\prime \prime}\right)_{t}$ is the unitary Radon-Nikodym cocycle for the vector state $\omega_{\xi_{1}}^{\prime \prime}$ of $\mathscr{M}^{\prime \prime}$ relative to the vector state $\omega_{\xi_{2}}^{\prime \prime}$ of $\mathscr{M}^{\prime \prime}$. To extend the above result to the $O_{D}^{*}$-algebra $(\mathscr{M}, \mathscr{D})$, we have to consider the following problems:

1. the extension of the modular automorphism groups $\left\{\sigma_{t}^{\xi_{1}}\right\}$ and $\left\{\sigma_{t}^{\xi_{2}}\right\}$ of $\mathscr{M}^{\prime \prime}$ to the $O_{p}^{*}$-algebra $(\mathscr{M}, \mathscr{D})$;

2. the invariance of domains under the unitary Radon-Nikodym cocycle $\left(D \omega_{\xi_{1}}^{\prime \prime}: D \omega_{\xi_{2}}^{\prime \prime}\right)_{t}$.

With this view, we define the following notion: A pair $\left(\xi_{1}, \xi_{2}\right)$ is said to be relative modular for $(\mathscr{M}, \mathscr{D})$ if there exists a subspace $\mathscr{E}$ of $\mathscr{D}$ such that $\xi_{1}, \xi_{2} \in \mathscr{E}, \mathscr{M} \mathscr{E}=\mathscr{E}, \Delta_{\xi_{1}}^{\prime i t} \mathscr{E}=\mathscr{E}$ and $\Delta_{\xi_{2}}^{\prime i t} \mathscr{E}=\mathscr{E}$ for all $t \in \mathbb{R}$, where $\Delta_{\xi_{1}}^{\prime \prime}$ and $\Delta_{\xi_{2}}^{\prime \prime}$ are modular operators of the left Hilbert algebras $\mathscr{M}^{\prime \prime} \xi_{1}$ and $\mathscr{M}^{\prime \prime} \xi_{2}$, respectively. Let $\left(\xi_{1}, \xi_{2}\right)$ be relative modular for $(\mathscr{M}, \mathscr{D})$. We denote by $\mathscr{D}_{\xi_{1} \xi_{2}}$ the maximal subspace in the set of the above subspaces $\mathscr{E}$ of $\mathscr{D}$, denote by $\left(\mathscr{M} / \mathscr{D}_{\xi_{1} \xi_{2}}\right)_{w c}^{\prime \prime}$ an unbounded bicommutant of the $O_{p}^{*}$-algebra $\left(\mathscr{M} / \mathscr{D}_{\xi_{1} \xi_{2}}, \mathscr{D}_{\xi_{1} \xi_{2}}\right)$ and put $\sigma_{t}^{\xi_{1}}(X)=$ $\Delta_{\xi_{1}^{\prime}}^{\prime \prime i t} X \Delta_{\xi_{1}}^{\prime \prime-i t}$ and $\sigma_{t}^{\xi_{2}}(X)=\Delta_{\xi_{2}}^{\prime \prime i t} X \Delta_{\xi_{2}}^{\prime \prime}$-it for $t \in \mathbb{R}$ and $X \in\left(\mathscr{M} / \mathscr{D}_{\xi_{1} \xi_{2}}\right)_{w c}^{\prime \prime}$. We show that the closed $O_{p}^{*}$-algebra $\left(\mathscr{M} / \mathscr{D}_{\xi_{1} \xi_{2}}\right)_{w c}^{\prime \prime}$ contains $\left(D \omega_{\xi_{\mathrm{N}}}^{\prime \prime}: D \omega_{\xi_{2}}^{\prime \prime}\right)_{t} / \mathscr{D}_{\xi_{1} \xi_{2}}$ for all $t \in \mathbf{R}$, and $\left\{\sigma_{t}^{\xi_{1}}\right\}$ and $\left\{\sigma_{t}^{\xi_{2}}\right\}$ are one-parameter groups of $*$-automorphisms of $\left(\mathscr{M} / \mathscr{D}_{\xi_{1} \xi_{2}}\right)$ wc which satisfy the relation: $\sigma_{t}^{\xi_{1}}(X) \xi=\left(D \omega_{\xi_{1}}^{\prime \prime}\right.$ : $\left.D \omega_{\xi_{2}}^{\prime \prime}\right)_{t} \sigma_{t}^{\xi_{2}}(X)\left(D \omega_{\xi_{1}}^{\prime \prime}: D \omega_{\xi_{2}}^{\prime \prime}\right)_{t}^{*} \xi$ for all $t \in \mathbf{R}, X \in\left(\mathscr{M} / \mathscr{D}_{\xi_{1} \xi_{2}}\right)_{w c}^{\prime \prime}$ and $\xi \in \mathscr{D}_{\xi_{1} \xi_{2}}$.

We study Radon-Nikodym theorems and Lebesgue decomposition theorems for $O_{p}^{*}$-algebras. Radon-Nikodym theorems for von Neumann algebras have been investigated in detail $[1,3,6,19,24,28$, 32]. In particular, in [19] Kosaki recently defined the notions of absolute continuity and singularity for normal forms on a von Neumann algebra $\mathscr{M}_{0}$ with a cyclic and separating vector $\xi_{0}$, and established a Lebesgue decomposition theorem. Further, he characterized strongly $\omega_{\xi_{0}}$-absolutely continuous (called $\omega_{\xi_{0}}$-absolutely continuous by Kosaki) forms and $\omega_{\xi_{0}}$-singular forms using the Tomita-Takesaki theory (modular operators, relative modular operators, unitary RadonNikodym cocycles etc).

On the other hand, in the case of $O_{p}^{*}$-algebras the study in this 
direction seems to be hardly done except for $[8,13,16]$. The difficulties in the case of $O_{p}^{*}$-algebras exist in the points that $\sigma$-weakly continuous positive linear functional on an $O_{p}^{*}$-algebra $\mathscr{M}$ is not necessarily a vector state and a pathological relation between the $O_{p}^{*}$-algebra $\mathscr{M}$ and the von Neumann algebra $\mathscr{M}^{\prime \prime}$ occures frequently.

In [8] Gudder defined the notion of strongly absolute continuity which is stronger than one of classical absolute continuity, and tried to obtain a Radon-Nikodym theorem for a $*$-algebra with no additional assumptions. Further, he defined the notion of singularity, and established a Lebesgue decomposition theorem in the Banach *-algebra case. After that, developing Gudder's results, in [13, 16] we obtained the following: Speaking roughly, a positive linear functional $\phi$ on a closed $O_{P}^{*}$-algebra $(\mathscr{M}, \mathscr{D})$ with a strongly cyclic vector $\xi_{0}$ is decomposed into the sum: $\phi=\phi_{c}+\phi_{s}$, where $\phi_{c}$ is a strongly $\omega_{\xi_{0}}$-absolutely continuous part of $\phi$ and $\phi_{s}$ is a $\omega_{\xi_{0}}$-singular part of $\phi$; and $\phi$ is strongly $\omega_{\xi_{0}}$-absolutely continuous if and only if $\phi=\phi_{c}$ if and only if $\phi$ is represented as $\phi=\omega_{H^{\prime} \xi_{0}}$ for some positive self-adjoint operator $H^{\prime}$ affiliated with $\mathscr{M}^{\prime}$ such that $\xi_{0} \in \mathscr{D}\left(H^{\prime}\right)$ and $H^{\prime} \xi_{0} \in \mathscr{D}$. However, we didn't know whether the strongly $\omega_{\xi_{0}}$-absolutely continuous part $\phi_{c}$ of $\phi$ in the above Lebesgue decomposition theorem is maximal, or not.

In Section 4 we show that Gudder's definitions of absolute continuity and singularity are identical with Kosaki's definitions, respectively, and apply Kosaki's results to the case of $O_{p}^{*}$-algebras. In particular, we obtain that a strongly $\omega_{\xi_{0}}$-absolutely continuous part $\phi_{c}$ in our Lebesgue decomposition theorem is maximal in the set of strongly $\omega_{\xi_{0}}$-absolutely continuous parts of $\phi$. Further, using an unbounded generalization of the Tomita-Takesaki theory developed in a previous paper [14] and Section 3, we generalize the RadonNikodym theorem of Pedersen and Takesaki [24] to the unbounded case.

In the case of $O_{p}^{*}$-algebras satisfying the von Neumann density type theorem, somewhat of the pathological facts for $O_{p}^{*}$-algebras are omitted, and so in Section 5 we obtain more detailed results for the Radon-Nikodym theorems, and further apply these results to the spatial theory for $O_{p}^{*}$-algebras. 
In Section 6 we first investigate the absolute continuity and the singularity of concrete positive linear functionals on the $O_{p}^{*}$-algebra $\mathscr{P}\left(-i \frac{d}{d t}\right)$ generated by the differential operator $-i \frac{d}{d t}$, and next characterize positive linear functionals on the canonical algebra $\mathscr{A}$ for one degree of freedom which are invariant with respect to the oneparameter group $\left\{\Delta_{\left\{e^{i n} \beta_{\}}\right.}^{i t}\right\}_{t \in \boldsymbol{R}}$ of $*$-automorphisms of $\mathscr{A}$ defined by [10], and finally by modifying Kosaki's examples [19] for von Neumann algebras we construct some concrete examples of positive linear functionals on the maximal $O_{p}^{*}$-algebra $\mathscr{L}^{\dagger}(\mathscr{S}(\mathbb{R}))$ on the Schwartz space $\mathscr{S}(\mathbb{R})$ which show that the sum of singular positive linear functionals need not be singular, the strongly absolute continuity is not hereditary and the Lebesgue decomposition is not necessarily unique.

\section{§ 2. Preliminaries}

In this section we review some of the definitions and the basic properties about $O_{p}^{*}$-algebras and refer to $[7,9,15,16,20,23,25$, 29] for further details.

Let $\mathscr{D}$ be a pre-Hilbert space with inner product $(\mid)$ and $\mathscr{S}(\mathscr{D})$ be the Hilbert space obtained by the completion of $\mathscr{D}$. We denote by $\mathscr{C}^{\dagger}(\mathscr{D}, \mathfrak{E}(\mathscr{D}))$ the set of all linear operators $X$ such that $\mathscr{D}(X) \cap \mathscr{D}\left(X^{*}\right) \supset \mathscr{D}$, and define a subset $\mathscr{L}^{\dagger}(\mathscr{D})$ of $\mathscr{C}^{\dagger}(\mathscr{D}, \mathscr{E}(\mathscr{D}))$ by

$$
\mathscr{L}^{\dagger}(\mathscr{D})=\left\{X \in \mathscr{C}^{\dagger}(\mathscr{D}, \mathscr{S}(\mathscr{D})) ; \mathscr{D}(X)=\mathscr{D}, X \mathscr{D} \subset \mathscr{D}, X^{*} \mathscr{D} \subset \mathscr{D}\right\} \text {. }
$$

Then $\mathscr{C}^{\dagger}\left(\mathscr{D}, \mathscr{S}_{\mathcal{C}}(\mathscr{D})\right)$ is a $*$-invariant vector space with the usual operations and the adjoint $X^{*}$, and $\mathscr{L}^{\dagger}(\mathscr{D})$ is a $*$-algebra with involution $X^{\dagger}=X^{*} / \mathscr{D}$. A $*$-subalgebra $\mathscr{M}$ of $\mathscr{L}^{\dagger}(\mathscr{D})$ is said to be an $O_{p}^{*}$-algebra on $\mathscr{D}$. We here treat with only $O_{p}^{*}$-algebras with identity operator $\mathbb{I}$. An $O_{p}^{*}$-algebra $\mathscr{M}$ on $\mathscr{D}$ is also denoted by $(\mathscr{M}, \mathscr{D})$.

Let $(\mathscr{M}, \mathscr{D})$ be an $O_{p}^{*}$-algebra. A locally convex topology on $\mathscr{D}$ defined by a family $\left\{\|\bullet\|_{X} ; X \in \mathscr{M}\right\}$ of seminorms:

$$
\|\xi\|_{X}=\|\xi\|+\|X \xi\|, \quad \xi \in \mathscr{D}
$$

is said to be the induced topology on $\mathscr{D}$, which is denoted by $t_{\mathscr{M}}$ 。 If $\left(\mathscr{D}, t_{\mathscr{M}}\right)$ is complete, then $(\mathscr{M}, \mathscr{D})$ is said to be closed. It follows 
from ([25] Lemma 2.6) that for each $O_{p}^{*}$-algebra $(\mathscr{M}, \mathscr{D})$ there exists a closed $O_{p}^{*}$-algebra $(\tilde{M}, \widetilde{\mathscr{D}})$ which is the smallest closed extension of $(\mathscr{M}, \mathscr{D})$, which is said to be the closure of $(\mathscr{M}, \mathscr{D})$. A vector $\xi_{0}$ in $\mathscr{D}$ is said to be cyclic (resp. strongly cyclic) for $\mathscr{M}$ if $\mathscr{M} \xi_{0}$ is dense in $\mathscr{S}(\mathscr{D})\left(\operatorname{resp} .\left(\mathscr{D}, t_{\mathscr{M}}\right)\right)$. If $\mathscr{D} \underset{X \in \mathscr{M}}{\bigcap} \mathscr{D}\left(X^{*}\right)$, then $(\mathscr{M}, \mathscr{D})$ is said to be self-adjoint.

We define some locally convex topologies on an $O_{p}^{*}$-algebra ( $\mathscr{M}$, $\mathscr{D})$. Locally convex topologies on $\mathscr{C}^{\dagger}(\mathscr{D}, \mathscr{L}(\mathscr{D}))$ defined by systems $\left\{P_{\xi, \eta}(\bullet) ; \xi, \eta \in \mathscr{D}\right\},\left\{P_{\xi}(\bullet) ; \xi \in \mathscr{D}\right\}$ and $\left\{P_{\xi}^{*}(\bullet) ; \xi \in \mathscr{D}\right\}$ of seminorms:

$$
P_{\xi, \eta}(X)=|(X \xi \mid \eta)|, P_{\xi}(X)=\|X \xi\|, P_{\xi}^{*}(X)=\|X \xi\|+\left\|X^{*} \xi\right\|
$$

are said to be a weak topology, a strong topology and a strong* topology, which are denoted by $t_{w}, t_{s}$ and $t_{s}^{*}$, respectively. To introduce $\sigma$-weak, $\sigma$-strong, $\sigma$-strong* topologies on $\mathscr{M}$, we define an $O_{p}^{*}$-algebra $\left([\mathscr{M}], \mathscr{D}^{\infty}(\mathscr{M})\right)$ as follows:

$$
\begin{aligned}
& \mathscr{D}^{\infty}(\mathscr{M})=\left\{\left\{\xi_{k}\right\} \subset \mathscr{D} ; \sum_{k=1}^{\infty}\left\|X \xi_{k}\right\|^{2}<\infty \text { for all } X \in \mathscr{M}\right\} ; \\
& {[X]\left\{\xi_{k}\right\}=\left\{X \xi_{k}\right\}, X \in \mathscr{M},\left\{\xi_{k}\right\} \in \mathscr{D}^{\infty}(\mathscr{M}) ;} \\
& {[\mathscr{M}]=\{[X] ; X \in \mathscr{M}\} .}
\end{aligned}
$$

The weakest locally convex topology on $\mathscr{M}$ such that the map $X \rightarrow[X]$ of $\mathscr{M}$ into $\left(\mathscr{C}^{\dagger}\left(\mathscr{D}^{\infty}(\mathscr{M}), \mathfrak{S}(\mathscr{D})^{\infty}\right), t_{w}\right)\left(\operatorname{resp} .\left(\mathscr{C}^{\dagger}\left(\mathscr{D}^{\infty}(\mathscr{M}), \mathfrak{S}(\mathscr{D})^{\infty}\right), t_{s}\right)\right.$, $\left.\left(\mathscr{C}^{\dagger}\left(\mathscr{D}^{\infty}(\mathscr{M}), \mathfrak{D}(\mathscr{D})^{\infty}\right), t_{s}^{*}\right)\right)$ is said to be a $\sigma$-weak (resp. $\sigma$-strong, $\sigma$-strong*) topology for $\mathscr{M}$, which is denoted by $t_{\sigma w}^{\mathscr{A}}\left(\right.$ resp. $\left.t_{\sigma s}^{\mathscr{M}}, t_{\sigma s}^{* \mathscr{M}}\right)$, where $\mathfrak{S}_{(\mathscr{D})^{\infty}}$ is the direct sum of the Hilbert spaces $\mathfrak{C}_{n}=\mathfrak{S}(\mathscr{D})$ for $n=1,2, \ldots$

We define commutants of an $O_{p}^{*}$-algebra $(\mathscr{M}, \mathscr{D})$ as follows:

$$
\begin{aligned}
\mathscr{M}^{\prime}=\{C \in \mathscr{B}(\mathfrak{D}(\mathscr{D})) & ;(C X \xi \mid \eta)=\left(C \xi \mid X^{\dagger} \eta\right) \\
& \text { for each } \xi, \eta \in \mathscr{D} \text { and } X \in \mathscr{M}\},
\end{aligned}
$$

where $\mathscr{B}(\mathscr{E}(\mathscr{D}))$ is the set of all bounded linear operators on $\mathscr{L}(\mathscr{D})$;

$$
\begin{aligned}
& \mathscr{M}_{\sigma}^{\prime}=\left\{S \in \mathscr{C}^{\dagger}(\mathscr{D}, \mathfrak{S}(\mathscr{D})) ;(X \xi \mid \mathrm{S} \eta)=\left(S^{*} \xi \mid X^{\dagger} \eta\right)\right. \\
& \qquad \text { for each } \xi, \eta \in \mathscr{D} \text { and } X \in \mathscr{M}\} ; \\
& \mathscr{M}_{c}^{\prime}=\mathscr{M}_{\sigma}^{\prime} \cap \mathscr{L}^{\dagger}(\mathscr{D}) .
\end{aligned}
$$

Then $\mathscr{M}_{w}^{\prime}$ (simply, $\mathscr{M}^{\prime}$ ) is a $*$-invariant weakly closed subspace of $\mathscr{B}(\mathscr{C}(\mathscr{D}))$, but it is not necessarily an algebra $[9,15,25]$. If $(\mathscr{M}, \mathscr{D})$ is self-adjoint, then $\mathscr{M}^{\prime} \mathscr{D}=\mathscr{D}$, which implies $\mathscr{M}^{\prime}$ is an algebra; and 
the converses don't necessarily hold. But, if $\mathscr{M}^{\prime}$ is an algebra, then there exists a closed $O_{p}^{*}$-algebra $(\hat{\mathscr{M}}, \hat{\mathscr{D}})$ which is the smallest extension of ( $\mathscr{M}, \mathscr{D}$ ) satisfying $\hat{\mathscr{M}}^{\prime}=\mathscr{M}^{\prime}$ and $\mathscr{M}^{\prime} \hat{\mathscr{D}}=\hat{\mathscr{D}}$ [16]. This result is a particular case of Proposition 5.5 in the Schmüdgen paper [29]. $\mathscr{M}_{\sigma}^{\prime}$ is a strongly* closed subspace of $\mathscr{C}^{\dagger}(\mathscr{D}, \mathfrak{S}(\mathscr{D}))$ whose bounded part is identical with $\mathscr{M}^{\prime}$; and $\mathscr{M}_{c}^{\prime}$ is an $O_{p}^{*}$-algebra on $\mathscr{D}$. We next define bicommutants of $\mathscr{M}$ as follows:

$$
\begin{aligned}
& \mathscr{M}^{\prime \prime} \equiv\left(\mathscr{M}_{w}^{\prime}\right)^{\prime}=\left\{A \in \mathscr{B}(\mathscr{D}(\mathscr{D})) ; \quad A C=C A \text { for each } C \in \mathscr{M}^{\prime}\right\} \\
& \mathscr{M}_{w \sigma}^{\prime \prime}=\left\{X \in \mathscr{C}^{\dagger}(\mathscr{D}, \mathscr{S}(\mathscr{D})) ; \quad(C X \xi \mid \eta)=\left(C \xi \mid X^{*} \eta\right)\right. \\
& \left.\quad \text { for each } \xi, \eta \in \mathscr{D} \text { and } C \in \mathscr{M}^{\prime}\right\}, \\
& \mathscr{M}_{w c}^{\prime \prime}=\mathscr{M}_{w o}^{\prime \prime} \cap \mathscr{L}^{\dagger}(\mathscr{D}) .
\end{aligned}
$$

Then $\mathscr{M}^{\prime \prime}$ is a von Neumann algebra on $\mathfrak{E}(\mathscr{D})$, but $\left(\mathscr{M}^{\prime \prime}\right)^{\prime}$ is not necessarily identical with $\mathscr{M}^{\prime}$. If $\mathscr{M}^{\prime}$ is an algebra, then $\left(\mathscr{M}^{\prime \prime}\right)^{\prime}=\mathscr{M}^{\prime}$. $\mathscr{M}_{w \sigma}^{\prime \prime}$ is a strongly* closed $*$-invariant subspace of $\mathscr{C}^{\dagger}(\mathscr{D}, \mathscr{S}(\mathscr{D}))$ containing $\mathscr{M} \cup \mathscr{M}^{\prime \prime}$ whose bounded part is identical with $\mathscr{M}^{\prime \prime}$; and $\mathscr{M}_{w c}^{\prime \prime}$ is an $O_{p}^{*}$-algebra on $\mathscr{D}$, which equals

$$
\mathscr{R}\left(\mathscr{M}^{\prime \prime}, \mathscr{D}\right) \equiv\left\{X \in \mathscr{L}^{\dagger}(\mathscr{D}) ; \bar{X} \text { is affiliated with } \mathscr{M}^{\prime \prime}\right\}
$$

if $\mathscr{M}^{\prime} \mathscr{D}=\mathscr{D}$. Further, $\mathscr{M}^{\prime}$ is an algebra if and only if the closure $\overline{\mathscr{M}}^{\prime \prime} t_{s}^{*}$ of $\mathscr{M}^{\prime \prime}$ in $\left(\mathscr{C}^{\dagger}(\mathscr{D}, \mathfrak{S}(\mathscr{D})), t_{s}^{*}\right)$ equals $\mathscr{M}_{w o}^{\prime \prime}$ if and only if $\overline{\mathscr{M}}^{\prime \prime} t_{s}^{*} \cap \mathscr{L}^{\dagger}(\mathscr{D})=\mathscr{M}_{w c}^{\prime \prime}[16]$.

A closed $O_{\mathscr{D}}^{*}$-algebra $(\mathscr{M}, \mathscr{D})$ is said to be a generalized von Neumann algebra if $\mathscr{M}^{\prime} \mathscr{D}=\mathscr{D}$ and $\mathscr{M}=\mathscr{M}_{w c .}^{\prime \prime}$ If $(\mathscr{M}, \mathscr{D})$ is a closed ${ }_{i} O_{p}^{*}$-algebra such that $\mathscr{M}^{\prime} \mathscr{D}=\mathscr{D}$, then $\mathscr{M}_{w c}^{\prime \prime}$ is a generalized von Neumann algebra.

Let $\mathscr{A}$ be a $*$-algebra. A $*$-homomorphism $\pi$ of $\mathscr{A}$ onto an $O_{p}^{*}-$ algebra on a dense subspace $\mathscr{D}(\pi)$ in a Hilbert space $\mathscr{S}(\pi)$ is said to be a $*$-representation of $\mathscr{A}$ in $\mathfrak{S}_{\pi}$ with domain $\mathscr{D}(\pi)$. Let $\pi$ be a $*$-representation of $\mathscr{A}$. We put

$$
\begin{aligned}
& \mathscr{D}(\tilde{\pi})=\underset{x \in \mathscr{A}}{\cap} \mathscr{D}(\overline{\pi(x)}), \tilde{\pi}(x) \xi=\overline{\pi(x)} \xi, x \in \mathscr{A}, \xi \in \mathscr{D}(\tilde{\pi}) ; \\
& \mathscr{D}\left(\pi^{*}\right)=\bigcap_{x \in \mathscr{A}} \mathscr{D}\left(\pi(x)^{*}\right), \pi^{*}(x) \xi=\pi\left(x^{*}\right) * \xi, x \in \mathscr{A}, \xi \in \mathscr{D}\left(\pi^{*}\right) .
\end{aligned}
$$

Then $\tilde{\pi}$ is a closed $*$-representation of $\mathscr{A}$ which is the smallest closed extension of $\pi$, which is said to be the closure of $\pi$, and $\pi^{*}$ is a closed representation of $\mathscr{A}$, but it is not necessarily a $*$-representation [9, 15, 25]. A *-representation $\pi$ of $\mathscr{A}$ is said to be closed (resp. self- 
adjoint) if $\pi=\tilde{\pi}$ (resp. $\left.\pi=\pi^{*}\right)$; that is, the $O_{p}^{*}$-algebra $(\pi(\mathscr{A}), \mathscr{D}(\pi)$ ) is closed (resp. self-adjoint).

Let $\phi$ be a positive linear functional on a $*$-algebra $\mathscr{A}$. It is easily shown that $\mathscr{N}_{\phi}=\left\{x \in \mathscr{A} ; \phi\left(x^{*} x\right)=0\right\}$ is a left ideal in $\mathscr{A}$. For each $x \in \mathscr{A}$ we denote by $\lambda_{\phi}(x)$ the coset of $\mathscr{A} / \mathscr{N}_{\phi}$ which contains $x$, and define an inner product $(\mid)$ on $\lambda_{\phi}(\mathscr{A})$ by

$$
\left(\lambda_{\phi}(x) \mid \lambda_{\phi}(y)\right)=\phi\left(y^{*} x\right), \quad x, y \in \mathscr{A} \text {. }
$$

Let $\mathfrak{S}_{\phi}$ be the Hilbert space which is completion of the pre-Hilbert space $\lambda_{\phi}(\mathscr{A})$, and $\pi_{\phi}$ be the closure of a $*$-representation $\pi_{\phi}^{0}$ of $\mathscr{A}$ defined by

$$
\pi_{\phi}^{0}(x) \lambda_{\phi}(y)=\lambda_{\phi}(x y), x, y \in \mathscr{A} \text {. }
$$

The triple $\left(\pi_{\phi}, \lambda_{\phi}, \mathfrak{C}_{\phi}\right)$ is said to be the GNS-construction for $\phi$ 。

\section{§3. Modular Vectors and Relative Modular Vectors}

In this section we first apply the unbounded Tomita-Takesaki theory developed in a previous paper [14] to the case of a closed $O_{p}^{*}$-algebra with a strongly cyclic and separating vector。

Throughout this section let $(\mathscr{M}, \mathscr{D})$ be a closed $O_{p}^{*}$-algebra such that $\mathscr{M}^{\prime} \mathscr{D}=\mathscr{D}$ and a vector $\xi_{0}$ in $\mathscr{D}$ be cyclic for $\mathscr{M}$ and separating for $\mathscr{M}^{\prime \prime}$. Since $\mathscr{M}^{\prime} \mathscr{D}=\mathscr{D}$, it follows that $\bar{X}$ is affiliated with $\mathscr{M}^{\prime \prime}$ for each $X \in \mathscr{M}$, which implies that $\xi_{0}$ is a cyclic vector for $\mathscr{M}^{\prime \prime}$, so that $\mathscr{M}^{\prime \prime} \xi_{0}$ is an achieved left Hilbert algebra in $\mathscr{S}(\mathscr{D})$ equipped with the multiplication $\left(A \xi_{0}\right)\left(B \xi_{0}\right)=A B \xi_{0}$ and the involution $A \xi_{0} \rightarrow A^{*} \xi_{0}$ 。 Let $S_{\xi_{0}}^{\prime \prime}$ be the closure of the involution $A \xi_{0} \rightarrow A^{*} \xi_{0}$ and

$$
S_{\xi_{0}}^{\prime \prime}=J_{\xi_{0}}^{\prime \prime} \Delta_{\xi_{0}}^{\prime \prime 1 / 2}
$$

be the polar decomposition of $S_{\xi_{0}}^{\prime \prime}$. The fundamental theorem of Tomita

$$
\begin{aligned}
& J_{\xi_{0}}^{\prime \prime} \mathscr{M}^{\prime \prime} J_{\xi_{0}}^{\prime \prime}=\mathscr{M}^{\prime}, \\
& \Delta_{\xi_{0}}^{\prime \prime i t} \mathscr{M}^{\prime \prime} \Delta_{\xi_{0}}^{\prime \prime-i t}=\mathscr{M}^{\prime \prime}, \quad \Delta_{\xi_{0}}^{\prime \prime i t} \mathscr{M}^{\prime} \Delta_{\xi_{0}}^{\prime \prime-i t}=\mathscr{M}^{\prime}, \quad t \in \mathbb{R}
\end{aligned}
$$

is obtained. Further, $\mathscr{M} \xi_{0}$ possesses the structure of an unbounded generalization of left Hilbert algebras; that is, $\mathscr{M} \xi_{0}$ is a dense subspace in $\mathscr{S}(\mathscr{D})$ and a $*$-algebra with the multiplication $\left(X \xi_{0}\right)\left(Y \xi_{0}\right)=X Y \xi_{0}$ and the closable involution $X \xi_{0} \rightarrow X^{\dagger} \xi_{0}$. Let $S_{\xi_{0}}$ be the closure of the involution $X \xi_{0} \rightarrow X^{\dagger} \xi_{0}$ and 


$$
S_{\xi_{0}}=J_{\xi_{0}} \Delta_{\xi_{0}}^{1 / 2}
$$

be the polar decomposition of $S_{\xi_{0}}$. Then, $S_{\xi_{0}} \subset S_{\xi_{0}}^{\prime \prime}$, but they don't necessarily equal. To extend (3.1) to the unbounded left Hilbert algebra $\mathscr{M} \xi_{0}$, we introduce the following notions:

Definition 3.1. A vector $\xi_{0}$ in $\mathscr{D}$ is said to be modular for $(\mathscr{M}, \mathscr{D})$ if the following conditions hold:

(1) $\xi_{0}$ is strongly cyclic for $\mathscr{M}$ and separating for $\mathscr{M}^{\prime \prime}$;

(2) there exists a subspace $\mathscr{E}$ of $\mathscr{D}$ such that $\mathscr{M} \xi_{0} \subset \mathscr{E} \subset \mathscr{D}$, $\mathscr{M} \mathscr{E}=\mathscr{E}$ and $\Delta_{\xi_{0}}^{\prime \prime i t} \mathscr{E}=\mathscr{E}$ for all $t \in \mathbb{R}$.

A modular vector $\xi_{0}$ for $(\mathscr{M}, \mathscr{D})$ is said to be standard if $S_{\xi_{0}}^{\prime \prime}=S_{\xi_{0}}$.

A positive linear functional $\phi$ on a $*$-algebra $\mathscr{A}$ with identity $e$ is said to be modular (resp. standard) if $\lambda_{\phi}(e)$ is a modular (resp. standard) vector for the $O_{p}^{*}$-algebra $\left(\pi_{\phi}(\mathscr{A}), \mathscr{D}\left(\pi_{\phi}\right)\right)$.

Let $\xi_{0}$ be a modular vector for $(\mathscr{M}, \mathscr{D})$. Put

$$
\begin{aligned}
& \mathscr{D}_{\xi_{0}}=\bigcup_{\mathscr{E} \in \mathscr{F}} \mathscr{E}, \\
& \mathscr{R}\left(\mathscr{M}^{\prime \prime}, \mathscr{D}_{\xi_{0}}\right)=\left\{X \in \mathscr{L}^{\dagger}\left(\mathscr{D}_{\xi_{0}}\right) ; \bar{X} \text { is affiliated with } \mathscr{M}^{\prime \prime}\right\},
\end{aligned}
$$

where $\mathscr{F}$ is the set of all subspaces $\mathscr{E}$ of $\mathscr{D}$ satisfying (1) and (2) of Definition 3.1. Then $\mathscr{D}_{\xi_{0}}$ is the largest element of $\mathscr{F}$.

By ([14] Theorem 3.3) we have the following

Theorem 3.2. Suppose $\xi_{0}$ is a modular vector for $(\mathscr{M}, \mathscr{D})$. Then the following statements hold.

(1) $\mathscr{R}\left(\mathscr{M}^{\prime \prime}, \mathscr{D}_{\xi_{0}}\right)$ is a generalized von Neumann algebra on $\mathscr{D}_{\xi_{0}}$, which equals the bicommutant $\left(\mathscr{M} / \mathscr{D}_{\xi_{0}}\right)_{w c}^{\prime \prime}$ of the $O_{p}^{*}$-algebra $\left(\mathscr{M} / \mathscr{D}_{\xi_{0}}, \mathscr{D}_{\xi_{0}}\right)$. In particular, if $(\mathscr{M}, \mathscr{D})$ is self-adjoint, then so is $\mathscr{R}\left(\mathscr{M}^{\prime \prime}, \mathscr{D}_{\xi_{0}}\right)$.

(2) Put

$$
\sigma_{t}^{\xi_{0}}(X)=\Delta_{\xi_{0}}^{\prime \prime i t} X \Delta_{\xi_{0}}^{\prime \prime-i t}, \quad X \in \mathscr{M}, \quad t \in \mathbf{R} .
$$

Then $\left\{\sigma_{t}^{\xi_{0}}\right\}_{t \in \mathbf{R}}$ is a one-parameter group of $*$-automorphisms of $\mathscr{R}\left(\mathscr{M}^{\prime \prime}, \mathscr{D}_{\xi_{0}}\right)$.

(3) The positive linear functional $\omega_{\xi_{0}}$ on $\mathscr{R}\left(\mathscr{M}^{\prime \prime}, \mathscr{D}_{\xi_{0}}\right)$ defined by

$$
\omega_{\xi_{0}}(X)=\left(X \xi_{0} \mid \xi_{0}\right), \quad X \in \mathscr{R}\left(\mathscr{M}^{\prime \prime}, \mathscr{D}_{\xi_{0}}\right)
$$


satisfies the $K M S$-condition with respect to $\left\{\sigma_{t}^{\xi_{0}}\right\}$; that is, for each $X, Y \in$ $\mathscr{R}\left(\mathscr{M}^{\prime \prime}, \mathscr{D}_{\xi_{0}}\right)$ there exists a function $f_{X, Y}$ in $A(0,1)$ such that

$$
f_{X, Y}(t)=\omega_{\xi_{0}}\left(\sigma_{t}^{\xi_{0}}(X) Y\right) \text { and } f_{X, Y}(t+i)=\omega_{\xi_{0}}\left(Y \sigma_{t}^{\xi_{0}}(X)\right)
$$

for all $t \in \mathbb{R}$, where $A(0,1)$ is the set of all complex-valued functions, bounded and continuous on $0 \leqq \mathbb{I}_{m} z \leqq 1$ and analytic in the interior.

Definition $3_{0} \mathcal{B}_{0}$ A system $\left(\mathscr{M}, \mathscr{D}, \xi_{0}\right)$ is said to be standard if the following conditions hold:

(1) $(\mathscr{M}, \mathscr{D})$ is a generalized von Neumann algebra;

(2) a vector $\xi_{0}$ in $\mathscr{D}$ is cyclic for $\mathscr{M}$ and separating for $\mathscr{M}^{\prime \prime}$;

(3) $\Delta_{\xi_{0}}^{\prime \prime i t} \mathscr{D}=\mathscr{D} \quad$ for all $t \in \mathbb{R}$.

A standard system $\left(\mathscr{M}, \mathscr{D}, \xi_{0}\right)$ is said to be full if $\xi_{0}$ is a strongly cyclic vector for $\mathscr{M}$.

Lemma $\mathfrak{B}_{0} 4_{0}$ (1) Suppose ( $\left.\mathscr{M}, \mathscr{D}, \xi_{0}\right)$ is a standard system. Then $\left\{\sigma_{t}^{\xi_{0}}\right\}$ is a one-parameter group of *-automorphisms of $\mathscr{M}$ and $\omega_{\xi_{0}}$ is a standard positive linear functional on $\mathscr{M}$ which satisfies the KMS-condition with respect to $\left\{\sigma_{t}^{\xi_{0}}\right\}$.

(2) Suppose $\left(\mathscr{M}, \mathscr{D}, \xi_{0}\right)$ is a full standard system. Then $\xi_{0}$ is a standard vector for $(\mathscr{M}, \mathscr{D})$ with $\mathscr{D}_{\xi_{0}}=\mathscr{D}$.

Proof. (1) It is clear that $\left\{\sigma_{t}^{\xi_{0}}\right\}$ is a one-parameter group of *-automorphisms of $\mathscr{M}$, which implies

$$
\Delta_{\xi_{0}}^{\prime \prime i t} \overline{\mathscr{M}}^{t}{ }^{\mathscr{L}}={\overline{\mathscr{M} \xi_{0}}}^{t} \mathscr{M}
$$

for all $t \in \mathbb{R}$, where $\overline{\mathscr{M}}_{0}^{t} \mathscr{M}$ denote the closure of $\mathscr{M} \xi_{0}$ relative to the induced topology $t_{\mathscr{H}}$. Hence, $\omega_{\xi_{0}}$ is a modular positive linear functional on $\mathscr{M}$ with $\mathscr{D}_{\omega_{\xi_{0}}}=\mathscr{D}\left(\pi_{\omega_{\xi_{0}}}\right)$. Further, it follows from ([14] Lemma 3.8) that $\triangle_{\xi_{0}}^{\prime i t}=\Delta_{\xi_{0}}^{i t}$ for all $t \in \mathbb{R}$, which implies $\omega_{\xi_{0}}$ is standard.

(2) This follows from (1).

Suppose $\xi_{0}$ is a modular vector for $(\mathscr{M}, \mathscr{D})$. By Theorem 3.2, $\left(\mathscr{R}\left(\mathscr{M}^{\prime \prime}, \mathscr{D}_{\xi_{0}}\right), \mathscr{D}_{\xi_{0}}, \xi_{0}\right)$ is a standard system, but it is not necessarily full。 
Lemma 3.5. Suppose $H$ is a positive self-adjoint operator in $\mathfrak{S}(\mathscr{D})$ affiliated with $\mathscr{M}^{\prime} \cap \mathscr{M}^{\prime \prime}$ such that $\xi_{0} \in \mathscr{D}(H)$ and $H \xi_{0} \in \mathscr{D}$. Then the following statements hold.

(1) Suppose $\xi_{0}$ is a modular vector for $(\mathscr{M}, \mathscr{D})$. Then $H \xi_{0} \in \mathscr{D}_{\xi_{0}}$ and the positive linear functional $\omega_{H \xi_{0}}$ on $\mathscr{R}\left(\mathscr{M}^{\prime \prime}, \mathscr{D}_{\xi_{0}}\right)$ satisfies the $K M S$ condition with respect to $\left\{\sigma_{t}^{\xi_{0}}\right\}$. Further, suppose $H$ is non-singular. Then $\left(\mathscr{R}\left(\mathscr{M}^{\prime \prime}, \mathscr{D}_{\xi_{0}}\right), \mathscr{D}_{\xi_{0}}, H \xi_{0}\right)$ is a standard system with $S_{H \xi_{0}}^{\prime \prime}=S_{\xi_{0}}^{\prime \prime}$

(2) Suppose $\left(\mathscr{M}, \mathscr{D}, \xi_{0}\right)$ is a standard system and $H$ is non-singular. Then $\left(\mathscr{M}, \mathscr{D}, H \xi_{0}\right)$ is a standard system. In particular, if $\left(\mathscr{M}, \mathscr{D}, \xi_{0}\right)$ is full, then so is $\left(\mathscr{M}, \mathscr{D}, H \xi_{0}\right)$.

Proof. (1) Since $\Delta_{\xi_{0}}^{\prime \prime i t} H \xi_{0}=H \xi_{0}$ for all $t \in \mathbf{R}$ and $\mathscr{D}_{\xi_{0}}$ is maximal, it follows that $H \xi_{0} \in \mathscr{D}_{\xi_{0}}$, so that the positive linear functional $\omega_{H \xi_{0}}$ on $\mathscr{R}\left(\mathscr{M}^{\prime \prime}, \mathscr{D}_{\xi_{0}}\right)$ is well-defined. By ([32] Theorem 15.4) the normal form $\omega_{H \xi_{0}}^{\prime \prime}$ on the von Neumann algebra $\mathscr{M}^{\prime \prime}$ defined by

$$
\omega_{H \xi_{0}}^{\prime \prime}(A)=\left(A H \xi_{0} \mid H \xi_{0}\right), \quad A \in \mathscr{M}^{\prime \prime}
$$

satisfies the KMS-condition with respect to $\left\{\sigma_{t}^{\xi_{0}}\right\}$. Hence, for each $A, B \in \mathscr{M}^{\prime \prime}$ there exists a function $f_{A, B} \in A(0,1)$ such that

$$
f_{A, B}(t)=\omega_{H \xi_{0}}^{\prime \prime}\left(\sigma_{t}^{\xi_{0}}(A) B\right), f_{A, B}(t+i)=\omega_{H \xi_{0}}^{\prime \prime}\left(B \sigma_{t}^{\xi_{0}}(A)\right)
$$

for all $t \in \mathbb{R}$. Since $\mathscr{R}\left(\mathscr{M}^{\prime \prime}, \mathscr{D}_{\xi_{0}}\right)^{\prime \prime}=\mathscr{M}^{\prime \prime}$ and $\mathscr{M}^{\prime} \mathscr{D}_{\xi_{0}}=\mathscr{D}_{\xi_{0}}$, it follows that for each $X, Y \in \mathscr{R}\left(\mathscr{M}^{\prime \prime}, \mathscr{D}_{\xi_{0}}\right)$ there exist sequences $\left\{A_{n}\right\},\left\{B_{n}\right\}$ in $\mathscr{M}^{\prime \prime}$ such that $\lim _{n \rightarrow \infty} A_{n} H \xi_{0}=X H \xi_{0}, \lim _{n \rightarrow \infty} A_{n}^{*} H \xi_{0}=X^{\dagger} H \xi_{0}, \lim _{n \rightarrow \infty} B_{n} H \xi_{0}=Y H \xi_{0}$ and $\lim _{n \rightarrow \infty} B_{n}^{*} H \xi_{0} \stackrel{n \rightarrow \infty}{=} Y^{\dagger} H \xi_{0}$. Then, since we have

$$
\begin{aligned}
& \sup _{t \in \mathbb{K}}\left|f_{A_{n^{B}} B_{n}}(t)-\left(\sigma_{t}^{\xi_{0}}(X) Y H \xi_{0} \mid H \xi_{0}\right)\right|=0, \\
& \sup _{t \in \mathbb{R}}\left|f_{A_{n}, B_{n}}(t+i)-\left(Y \sigma_{t}^{\xi_{0}}(X) H \xi_{0} \mid H \xi_{0}\right)\right|=0,
\end{aligned}
$$

it follows that there exists a function $f_{X, Y} \in A(0,1)$ such that

$$
f_{X, Y}(t)=\omega_{H \xi_{0}}\left(\sigma_{t}^{\xi_{0}}(X) Y\right), \quad f_{X, Y}(t+i)=\omega_{H \xi_{0}}\left(Y \sigma_{t}^{\xi_{0}}(X)\right)
$$

for all $t \in \mathbf{R}$; that is, $\omega_{H \xi_{0}}$ satisfies the KMS-condition with respect to $\left\{\sigma_{t}^{\xi_{0}}\right\}$.

Suppose $H$ is non-singular. Then it is clear that $H \xi_{0}$ is cyclic 
and separating for $\mathscr{R}\left(\mathscr{M}^{\prime \prime}, \mathscr{D}_{\xi_{0}}\right)^{\prime \prime}=\mathscr{M}^{\prime \prime}$. Let $H=\int_{0}^{\infty} \lambda d E(\lambda)$ be the spectral resolution of $H$ and put

$$
H_{n}=\int_{0}^{n} \lambda d E(\lambda), K_{n}=\int_{1 / n}^{n} \frac{1}{\lambda} d E(\lambda), E_{n}=\int_{1 / n}^{n} d E(\lambda), n \in \mathbb{N} .
$$

Since $H_{n}, K_{n}, E_{n} \in \mathscr{M}^{\prime} \cap \mathscr{M}^{\prime \prime}$, it follows that their restrictions to $\mathscr{D}_{\xi_{0}}$ are contained in $\mathscr{R}\left(\mathscr{M}^{\prime \prime}, \mathscr{D}_{\xi_{0}}\right)$. Since $H$ is non-singular, it follows that $\left\{E_{n}\right\}$ converges strongly to $I$, which implies

$$
\lim _{n \rightarrow \infty} K_{n} X H \xi_{0}=\lim _{n \rightarrow \infty} E_{n} X \xi_{0}=X \xi_{0}
$$

for each $X \in \mathscr{R}\left(\mathscr{M}^{\prime \prime}, \mathscr{D}_{\xi_{0}}\right)$, so that $H \xi_{0}$ is cyclic for $\mathscr{R}\left(\mathscr{M}^{\prime \prime}, \mathscr{D}_{\xi_{0}}\right)$. Further, we have

$$
\begin{aligned}
& \lim _{n \rightarrow \infty} A K_{n} H \xi_{0}=\lim _{n \rightarrow \infty} E_{n} A \xi_{0}=A \xi_{0}, \\
& \lim _{n \rightarrow \infty} K_{n} A^{*} H \xi_{0}=\lim _{n \rightarrow \infty} K_{n} H A^{*} \xi_{0}=A^{*} \xi_{0}, \\
& \lim _{n \rightarrow \infty} A H_{n} \xi_{0}=A H \xi_{0}, \lim _{n \rightarrow \infty} A^{*} H_{n} \xi_{0}=A^{*} H \xi_{0}
\end{aligned}
$$

for each $A \in \mathscr{M}^{\prime \prime}$. Hence, $S_{H \xi_{0}}^{\prime \prime}=S_{\xi_{0}}^{\prime \prime}$, and so $\Delta_{H \xi_{0}}^{\prime \prime i t} \mathscr{D}_{\xi_{0}}=\Delta_{\xi_{0}}^{\prime \prime i t} \mathscr{D}_{\xi_{0}}=\mathscr{D}_{\xi_{0}}$ for all $t \in \mathbb{R}$. Thus $\left(\mathscr{R}\left(\mathscr{M}^{\prime \prime}, \mathscr{D}_{\xi_{0}}\right), \mathscr{D}_{\xi_{0}}, H \xi_{0}\right)$ is a standard system.

(2) It follows from (1) that if $\left(\mathscr{M}, \mathscr{D}, \xi_{0}\right)$ is a standard system, then so is $\left(\mathscr{M}, \mathscr{D}, H \xi_{0}\right)$. Suppose $\left(\mathscr{M}, \mathscr{D}, \xi_{0}\right)$ is full. For each $X \in \mathscr{M}$ we have

$$
\lim _{n \rightarrow \infty} K_{n} X H \xi_{0}=X \xi_{0} \text { and } \lim _{n \rightarrow \infty} Y K_{n} X H \xi_{0}=Y X \xi_{0}
$$

for each $Y \in \mathscr{M}$. Hence, $H \xi_{0}$ is a strongly cyclic vector for $\mathscr{M}$. Thus, $\left(\mathscr{M}, \mathscr{D}, H \xi_{0}\right)$ is full.

To apply the unitary Radon-Nikodym cocycle introduced by Connes [3] to unbounded operator algebras, we define the following notion.

Definition 3.6. Let $(\mathscr{M}, \mathscr{D})$ be a closed $O_{p}^{*}$-algebra. A pair $\left(\xi_{1}, \xi_{2}\right)$ of vectors in $\mathscr{D}$ is said to be relative modular for $(\mathscr{M}, \mathscr{D})$ if the following conditions hold:

(1) $\xi_{1}$ and $\xi_{2}$ are strongly cyclic for $\mathscr{M}$ and separating for $\mathscr{M}^{\prime \prime}$;

(2) there exists a subspace $\mathscr{E}$ of $\mathscr{D}$ such that

(a) $\xi_{1}, \xi_{2} \in \mathscr{E}$; 
(b) $\mathscr{M} \mathscr{E}=\mathscr{E}$;

(c) $\Delta_{\xi_{1}}^{\prime \prime i t} \mathscr{E}=\mathscr{E}$ and $\Delta_{\xi_{2}}^{\prime \prime i t} \mathscr{E}=\mathscr{E}$

for all $t \in \mathbb{R}$.

Lemma 3.7. Let $(\mathscr{M}, \mathscr{D})$ be a closed $O_{p}^{*}$-algebra such that $\mathscr{M}^{\prime} \mathscr{D}=\mathscr{D}$ and a pair $\left(\xi_{1}, \xi_{2}\right)$ in $\mathscr{D}$ be relative modular for $(\mathscr{M}, \mathscr{D})$. Then the following statements hold.

(1) Put

$$
\mathscr{D}_{\xi_{1} \xi_{2}}=\bigcup_{\mathscr{E} \in \mathscr{F}} \mathscr{E},
$$

where $\mathscr{F}$ is the set of all subspaces $\mathscr{E}$ of $\mathscr{D}$ satisfying (a), (b) and (c) of Definition 3.6. Then $\mathscr{D}_{\xi_{1} \xi_{2}}$ is maximal in $\mathscr{F}$.

(2) $\xi_{1}$ and $\xi_{2}$ are modular vectors for $(\mathscr{M}, \mathscr{D})$ satisfying $\mathscr{D}_{\xi_{1} \xi_{2}} \subset \mathscr{D}_{\xi_{1}}$ $\cap \mathscr{D}_{\xi_{2}}$ 。

(3) $\mathscr{M}^{\prime} \mathscr{D}_{\xi_{1} \xi_{2}}=\mathscr{D}_{\xi_{1} \xi_{2}}$

(4) Put

$$
\mathscr{R}\left(\mathscr{M}^{\prime \prime}, \mathscr{D}_{\xi_{1} \xi_{2}}\right)=\left\{X \in \mathscr{L}^{\dagger}\left(\mathscr{D}_{\xi_{1} \xi_{2}}\right) ; \bar{X} \text { is affliated with } \mathscr{M}^{\prime \prime}\right\} \text {. }
$$

Then $\mathscr{R}\left(\mathscr{M}^{\prime \prime}, \mathscr{D}_{\xi_{1} \xi_{2}}\right)$ is a generalized von Neumann algebra on $\mathscr{D}_{\xi_{1} \xi_{2}}$ such that $\mathscr{R}\left(\mathscr{M}^{\prime \prime}, \mathscr{D}_{\xi_{1} \xi_{2}}\right)^{\prime}=\mathscr{M}^{\prime} . \quad$ In particular, if $(\mathscr{M}, \mathscr{D})$ is self-adjoint, then $\left(\mathscr{R}\left(\mathscr{M}^{\prime \prime}, \mathscr{D}_{\xi_{1} \xi_{2}}\right), \mathscr{D}_{\xi_{1} \xi_{2}}\right)$ is self-adjoint.

(5) Put

$$
\sigma_{t}^{\xi_{1}}(X)=\Delta_{\xi_{1}}^{\prime \prime i t} X \Delta_{\xi_{1}}^{\prime \prime}-i t, \quad \sigma_{t}^{\xi_{2}}(X)=\Delta_{\xi_{2}}^{\prime \prime i t} X \Delta_{\xi_{2}}^{\prime \prime}-i t
$$

for $X \in \mathscr{R}\left(\mathscr{M}^{\prime \prime}, \mathscr{D}_{\xi_{1} \xi_{2}}\right)$ and $t \in \mathbb{R}_{\text {. }} \quad$ Then $\left\{\sigma_{t}^{\xi_{1}}\right\}_{t \in \mathbb{R}}$ and $\left\{\sigma_{t}^{\xi_{2}}\right\}_{t \in \boldsymbol{R}}$ are oneparameter groups of *-automorphisms of the generalized von Neumann algebra $\mathscr{R}\left(\mathscr{M}^{\prime \prime}, \mathscr{D}_{\xi_{1} \xi_{2}}\right)$.

(6) $\quad\left(\mathscr{R}\left(\mathscr{M}^{\prime \prime}, \mathscr{D}_{\xi_{1} \xi_{2}}\right), \mathscr{D}_{\xi_{1} \xi_{2}}, \xi_{1}\right)$ and $\left(\mathscr{R}\left(\mathscr{M}^{\prime \prime}, \mathscr{D}_{\xi_{1} \xi_{2}}\right), \mathscr{D}_{\xi_{1} \xi_{2}}, \xi_{2}\right)$ are standard systems.

Proof. The statements (1) and (2) are trivial.

(3) It is easily shown that the subspace generated by $\mathscr{M}^{\prime} \mathscr{D}_{\xi_{1} \xi_{2}}$ satisfies the conditions (1), (2) and (3) of Definition 3.6. Since $\mathscr{D}_{\xi_{1} \xi_{2}}$ is maximal, we have $\mathscr{M}^{\prime} \mathscr{D}_{\xi_{1} \xi_{2}}=\mathscr{D}_{\xi_{1} \xi_{2}}$

(4) Since $\mathscr{M} \xi_{1} \subset \mathscr{D}_{\xi_{1} \xi_{2}} \subset \mathscr{D}$, we have $\left(\mathscr{M} / \mathscr{D}_{\dot{\xi}_{1} \xi_{2}}\right)^{\prime}=\mathscr{M}^{\prime}$. It hence 
follows from (3) that $\mathscr{R}\left(\mathscr{M}^{\prime \prime}, \mathscr{D}_{\xi_{1} \xi_{2}}\right)$ is an $O_{p}^{*}$-algebra on $\mathscr{D}_{\xi_{1} \xi_{2}}$ containing $\mathscr{M} / \mathscr{D}_{\xi_{1} \xi_{2}}$ such that

$$
\left\{\begin{array}{l}
\mathscr{R}\left(\mathscr{M}^{\prime \prime}, \mathscr{D}_{\xi_{1} \xi_{2}}\right)^{\prime}=\mathscr{M}^{\prime}, \\
\Delta_{\xi_{1}^{\prime \prime}}^{\prime i t} \mathscr{R}\left(\mathscr{M}^{\prime \prime}, \mathscr{D}_{\xi_{1} \xi_{2}}\right) \Delta_{\xi_{1}}^{\prime \prime-i t}=\mathscr{R}\left(\mathscr{M}^{\prime \prime}, \mathscr{D}_{\xi_{1} \xi_{2}}\right), \\
\Delta_{\xi_{2}}^{\prime \prime i t} \mathscr{R}\left(\mathscr{M}^{\prime \prime}, \mathscr{D}_{\xi_{1} \xi_{2}}\right) \Delta_{\xi_{2}}^{\prime \prime-i t}=\mathscr{R}\left(\mathscr{M}^{\prime \prime}, \mathscr{D}_{\xi_{1} \xi_{2}}\right), t \in \mathbb{R} .
\end{array}\right.
$$

Put

$$
\widetilde{\mathscr{D}}_{\xi_{1} \xi_{2}}=\underset{X \in \mathscr{R}\left(\mathscr{M}^{\prime \prime}, \mathscr{D}_{\xi_{1} \xi_{2}}\right)}{\cap} \mathscr{D}(\bar{X}), \mathscr{D}_{\xi_{1} \xi_{2}}^{*}=\underset{X \in \mathscr{R}\left(\mathscr{M}^{\prime \prime}, \mathscr{D}_{\xi_{1} \xi_{2}}\right)}{\bigcap} \mathscr{D}\left(X^{*}\right)
$$

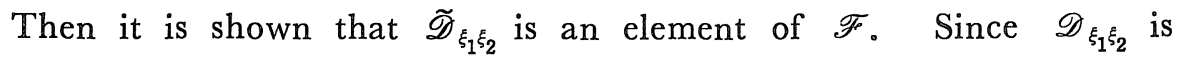
maximal, it follows that $\widetilde{\mathscr{D}}_{\xi_{1} \xi_{2}}=\mathscr{D}_{\xi_{1} \xi_{2}} ;$ that is, $\left(\mathscr{R}\left(\mathscr{M}^{\prime \prime}, \mathscr{D}_{\xi_{1} \xi_{2}}\right), \mathscr{D}_{\xi_{1} \xi_{2}}\right)$ is closed. Thus, $\left(\mathscr{R}\left(\mathscr{M}^{\prime \prime}, \mathscr{D}_{\xi_{1} \xi_{2}}\right), \mathscr{D}_{\xi_{1} \xi_{2}}\right)$ is a generalized von Neumann algebra. Suppose $(\mathscr{M}, \mathscr{D})$ is self-adjoint. Then it is shown that $\mathscr{D}_{\xi_{1} \xi_{2}}^{*}$ is an element of $\mathscr{F}$, which implies $\left(\mathscr{R}\left(\mathscr{M}^{\prime \prime}, \mathscr{D}_{\xi_{1} \xi_{2}}\right), \mathscr{D}_{\xi_{1} \xi_{2}}\right)$ is self-adjoint.

(5) This follows from $(3.2)$

(6) This follows from (3) and (4).

Let $(\mathscr{M}, \mathscr{D})$ be a closed $O_{p}^{*}$-algebra such that $\mathscr{M}^{\prime} \mathscr{D}=\mathscr{D}$ and vectors $\xi_{1}$ and $\xi_{2}$ in $\mathscr{D}$ be strongly cyclic for $\mathscr{M}$ and separating for $\mathscr{M}^{\prime \prime}$. Let $\mathfrak{S}_{4}$ be a four-dimensional Hilbert space with an orthogonal basis $\left\{\eta_{i j}\right\}_{i, j=1,2}$ and $\mathscr{F}_{2}$ be a $2 \times 2$-matrix algebra generated by the matrices $E_{i j}$ which are defined by $E_{i j} \eta_{k l}=\delta_{j k} \eta_{i l}$. Then we have the following

Lemma 3.8. $\mathscr{M} \otimes \mathscr{F}_{2}$ is a closed $O_{p}^{*}$-algebra on $\mathscr{D} \otimes \mathfrak{S}_{4}$ such that $\left(\mathscr{M} \otimes \mathscr{F}_{2}\right)^{\prime}\left(\mathscr{D} \otimes \mathfrak{S}_{4}\right)=\mathscr{D} \otimes \mathfrak{S}_{4}$, and a vector $\Omega_{\xi_{1} \xi_{2}} \equiv \xi_{1} \otimes \eta_{11}+\xi_{2} \otimes \eta_{22}$ in $\mathscr{D} \otimes \mathfrak{S}_{4}$ is strongly cyclic for $\mathscr{M} \otimes \mathscr{F}_{2}$ and separating for $\left(\mathscr{M} \otimes \mathscr{F}_{2}\right)^{\prime \prime}$ 。

Theorem 3.9. Let $(\mathscr{M}, \mathscr{D})$ be a closed $O_{p}^{*}$-algebra such that $\mathscr{M}^{\prime} \mathscr{D}$ $=\mathscr{D}$, and vectors $\xi_{1}$ and $\xi_{2}$ in $\mathscr{D}$ be strongly cyclic for $\mathscr{M}$ and separating for $\mathscr{M}^{\prime \prime}$. Then the following statements hold.

I. A pair $\left(\xi_{1}, \xi_{2}\right)$ in $\mathscr{D}$ is relative modular for $(\mathscr{M}, \mathscr{D})$ if and only if $\Omega_{\xi_{1} \xi_{2}}$ is a modular vector for $\left(\mathscr{M} \otimes \mathscr{F}_{2}, \mathscr{D} \otimes \mathscr{S}_{4}\right)$. In this case, $\mathscr{D}_{\Omega_{\xi_{1} \xi_{2}}}=$ $\mathscr{D}_{\xi_{1} \xi_{2}} \otimes \mathfrak{S}_{4}$.

II. Suppose that $\left(\xi_{1}, \xi_{2}\right)$ is relative modular for $(\mathscr{M}, \mathscr{D})$. Then 
(1) $\quad\left(D \omega_{\xi_{1}}^{\prime \prime}: D \omega_{\xi_{2}}^{\prime \prime}\right) t \mathscr{D}_{\xi_{1} \xi_{2}} \in \mathscr{R}\left(\mathscr{M}^{\prime \prime}, \mathscr{D}_{\xi_{1} \xi_{2}}\right)$ for all $t \in \mathbf{R}$, where $\left(D \omega_{\xi_{1}}^{\prime \prime}\right.$ : $\left.D \omega_{\xi_{2}}^{\prime \prime}\right)_{t}$ denotes the unitary Radon-Nikodym cocycle of the normal form $\omega_{\xi_{1}}^{\prime \prime}$ of $\mathscr{M}^{\prime \prime}$ relative to the normal form $\omega_{\xi_{2}}^{\prime \prime}$ of $\mathscr{M}^{\prime \prime}$;

(2) $\sigma_{t}^{\xi_{1}}(X) \xi=\left(D \omega_{\xi_{1}}^{\prime \prime}: D \omega_{\xi_{2}}^{\prime \prime}\right){ }_{t} \sigma_{t}^{\xi_{2}}(X)\left(D \omega_{\xi_{1}}^{\prime \prime}: D \omega_{\xi_{2}}^{\prime \prime}\right)_{t}^{*} \xi$ for all $t \in \mathbb{R}, X \in$ $\mathscr{R}\left(\mathscr{M}^{\prime \prime}, \mathscr{D}_{\xi_{1} \xi_{2}}\right)$ and $\xi \in \mathscr{D}_{\xi_{1} \xi_{2}}$.

Proof. I. Suppose $\left(\xi_{1}, \xi_{2}\right)$ is relative modular for $(\mathscr{M}, \mathscr{D})$. Since $\xi_{1}, \xi_{2} \in \mathscr{D}_{\xi_{1} \xi_{2}}$ and $\mathscr{M}_{\xi_{1} \xi_{2}}=\mathscr{D}_{\xi_{1} \xi_{2}}$, it follows that $\Omega_{\xi_{1} \xi_{2}} \in \mathscr{D}_{\xi_{1} \xi_{2}} \otimes \mathscr{S}_{4}$ and $\left(\mathscr{M} \otimes \mathscr{F}_{2}\right)\left(\mathscr{D}_{\xi_{1} \xi_{2}} \otimes \mathscr{S}_{4}\right)=\mathscr{D}_{\xi_{1} \xi_{2}} \otimes \mathscr{S}_{4} . \quad$ To show $\Delta_{\mathscr{O}_{\xi_{1} \xi_{2}}^{\prime \prime i t}}\left(\mathscr{D}_{\xi_{1} \xi_{2}} \otimes \mathscr{S}_{4}\right)=\mathscr{D}_{\xi_{1} \xi_{2}} \otimes \mathscr{S}_{4}$ for all $t \in \mathbf{R}$, we here state about the definition and the basic properties of the relative modular operators [2]. Let $\xi$ and $\eta$ be cyclic and separating vectors for the von Neumann algebra $\mathscr{M}^{\prime \prime}$. Let $S_{\xi \eta}^{\prime \prime}$ denote the closure of the conjugate linear operator on $\mathscr{M}^{\prime \prime} \eta$ defined by

$$
S_{\xi \eta}^{\prime \prime} A \eta=A^{*} \xi, \quad A \in \mathscr{M}^{\prime \prime}
$$

and let

$$
S_{\xi \eta}^{\prime \prime}=J_{\xi \eta}^{\prime \prime} \Delta_{\xi \eta}^{\prime \prime 1 / 2}
$$

denote the polar decomposition of $S_{\xi \eta}^{\prime \prime}$. The positive selfadjoint operator $\Delta_{\xi \eta}^{\prime \prime}=S_{\xi \eta}^{\prime \prime *} S_{\xi \eta}^{\prime \prime}$ is called the relative modular operator of $\xi$ and $\eta$. The relative modular operators satisfy the following properties [2]:

$$
\Delta_{\xi \eta}^{\prime \prime i t} A \Delta_{\xi \eta}^{\prime \prime-i t}=\sigma_{t}^{\xi}(A), \quad A \in \mathscr{M}^{\prime \prime}, \quad t \in \mathbf{R} ;
$$

for each cyclic and separating vector $\zeta$ for $\mathscr{M}^{\prime \prime}$. By (3.4) and Lemma 3. 7 we have

$$
\begin{aligned}
& \Delta_{\xi_{1} \xi_{2}}^{\prime \prime i t} \mathscr{D}_{\xi_{1} \xi_{2}}=\Delta_{\xi_{1} \xi_{2}}^{\prime \prime i t} \Delta_{\xi_{1}}^{\prime \prime-i t} \Delta_{\xi_{1}}^{\prime \prime i t} \mathscr{D}_{\xi_{1} \xi_{2}} \\
& \subset \mathscr{M}^{\prime} \mathscr{D}_{\xi_{1} \xi_{2}}=\mathscr{D}_{\xi_{1} \xi_{2}}, \\
& \|_{\xi_{2} \xi_{1}}^{\prime \prime i t} \mathscr{D}_{\xi_{1} \xi_{2}} \subset \mathscr{D}_{\hat{\xi}_{1} \xi_{2}}, \quad t \in \mathbf{R} \text {. }
\end{aligned}
$$

Since

$$
\begin{aligned}
& \Delta_{\Omega_{\xi_{1} \xi_{2}}^{\prime \prime}}^{\prime \prime i t}\left(\zeta_{1} \otimes \eta_{11}+\zeta_{2} \otimes \eta_{21}+\zeta_{3} \otimes \eta_{12}+\zeta_{4} \otimes \eta_{22}\right) \\
& \quad=\Delta_{\xi_{1}}^{\prime \prime i t} \zeta_{1} \otimes \eta_{11}+\Delta_{\xi_{2} \xi_{1}}^{\prime \prime i t} \zeta_{2} \otimes \eta_{21}+\Delta_{\xi_{1} \xi_{2}}^{\prime \prime i t} \zeta_{3} \otimes \eta_{12}+\Delta_{\xi_{2}}^{\prime \prime i t} \zeta_{4} \otimes \eta_{22}
\end{aligned}
$$

for all $\zeta_{1}, \zeta_{2}, \zeta_{3}, \zeta_{4} \in \mathscr{D}_{\xi_{1} \xi_{2}}$ and $t \in \mathbb{R}$, it follows from (3.6) that 


$$
\Delta_{\Omega_{\xi_{1} \xi_{2}}^{\prime \prime i t}}\left(\mathscr{D}_{\xi_{1} \xi_{2}} \otimes \mathcal{S}_{4}\right)=\mathscr{D}_{\xi_{1} \xi_{2}} \otimes \mathcal{S}_{4}, \quad t \in \mathbb{R},
$$

which implies that $\Omega_{\xi_{1} \xi_{2}}$ is a modular vector for $\left(\mathscr{M} \otimes \mathscr{F}_{2}, \mathscr{D}_{\xi_{1} \xi_{2}} \otimes \mathfrak{S}_{4}\right)$ with

$$
\mathscr{D}_{\xi_{1} \xi_{2}} \otimes \mathscr{S}_{4} \subset \mathscr{D}_{\Omega_{\xi_{1} \xi_{2}}}
$$

Suppose $\Omega_{\xi_{1} \xi_{2}}$ is a modular vector for $\left(\mathscr{M} \otimes \mathscr{F}_{2}, \mathscr{D} \otimes \mathcal{S}_{4}\right)$. Put

$$
\mathscr{E}=\left\{\zeta_{1} \in \mathscr{D} ; \zeta_{1} \otimes \eta_{11}+\zeta_{2} \otimes \eta_{21}+\zeta_{3} \otimes \eta_{12}+\zeta_{4} \otimes \eta_{22} \in \mathscr{D}_{\Omega_{\xi_{1} \xi_{2}}}\right\}_{\text {. }}
$$

Identifying

$$
\zeta=\zeta_{1} \otimes \eta_{11}+\zeta_{2} \otimes \eta_{21}+\zeta_{3} \otimes \eta_{12}+\zeta_{4} \otimes \eta_{22} \in S_{\mathcal{C}} \otimes S_{4}
$$

with $\zeta=\left(\zeta_{1}, \zeta_{2}, \zeta_{3}, \zeta_{4}\right) \in \mathfrak{S} \oplus \mathcal{S} \oplus \mathscr{C} \oplus \mathcal{C}$, every element $X=\sum_{i, j=1}^{2} X_{i j} \otimes E_{i j} \in \mathscr{M}$ $\bigotimes \mathscr{F}_{2}$ is represented as the following matrix

$$
X=\left|\begin{array}{llll}
X_{11} & X_{12} & 0 & 0 \\
X_{21} & X_{22} & 0 & 0 \\
0 & 0 & X_{11} & X_{12} \\
0 & 0 & X_{21} & X_{22}
\end{array}\right|
$$

Further, it is clear that

$$
\left(\mathscr{M} \otimes \mathscr{F}_{2}\right)^{\prime}=\left\{\left(\begin{array}{llll}
C_{11} & 0 & C_{12} & 0 \\
0 & C_{11} & 0 & C_{12} \\
C_{21} & 0 & C_{22} & 0 \\
0 & C_{21} & 0 & C_{22}
\end{array}\right) ; G_{i j} \in \mathscr{M}^{\prime}, i, j=1,2\right\} 。
$$

Since $\left(\mathscr{M} \otimes \mathscr{F}_{2}\right) \mathscr{D}_{\Omega_{\xi_{1} \xi_{2}}}=\mathscr{D}_{\Omega_{\xi_{1} \xi_{2}}}$ and $\left(\mathscr{M} \otimes \mathscr{F}_{2}\right)^{\prime} \mathscr{D}_{\Omega_{\xi_{1} \xi_{2}}}=\mathscr{D}_{\Omega_{\xi_{1} \xi_{2}}}$, it follows that

$$
\zeta_{i} \in \mathscr{E} \quad(i=1,2,3,4)
$$

for each $\zeta=\left(\zeta_{1}, \zeta_{2}, \zeta_{3}, \zeta_{4}\right) \in \mathscr{D}_{\Omega_{\xi_{1} \xi_{2}}}$, which implies that $\xi_{1}, \xi_{2} \in \mathscr{E}, \mathscr{M} \mathscr{E}=\mathscr{E}$, and $\Delta_{\xi_{1}}^{\prime \prime i t} \mathscr{E}=\mathscr{E}$, and $\Delta_{\xi_{2}}^{\prime \prime i t} \mathscr{E}=\mathscr{E}$ for all $t \in \mathbb{R}$, so that $\left(\xi_{1}, \xi_{2}\right)$ is relative modular for $(\mathscr{M}, \mathscr{D})$ with $\mathscr{E} \subset \mathscr{D}_{\xi_{1} \xi_{2}{ }^{\circ}}$ Hence, by (3.7) and (3.8) we have

$$
\mathscr{D}_{\xi_{1} \xi_{2}} \bigotimes \mathscr{S}_{4} \subset \mathscr{D}_{\Omega_{\xi_{1} \xi_{2}}} \subset \mathscr{E} \otimes \mathscr{S}_{4} \subset \mathscr{D}_{\xi_{1} \xi_{2}} \bigotimes \mathscr{S}_{4} \text { 。 }
$$

II. By (3.5) we have

$$
\left(D \omega_{\xi_{1}}^{\prime \prime}: D \omega_{\xi_{2}}^{\prime \prime}\right)_{t}=\Delta_{\xi_{1}}^{\prime \prime i t} \Delta_{\xi_{2}}^{\prime \prime} \xi_{1}^{-i t}=\Delta_{\xi_{1} \xi_{2}}^{\prime \prime i t} \Delta_{\xi_{2}}^{\prime \prime-i t}, \quad t \in \mathbb{R} \text {. }
$$


It hence follows that

$$
\begin{aligned}
\left(D \omega_{\xi_{1}}^{\prime \prime}: D \omega_{\xi_{2}}^{\prime \prime}\right)_{t} \mathscr{D}_{\xi_{1} \xi_{2}} & =\Delta_{\xi_{1}}^{\prime \prime i t} \Delta_{\xi_{2} \xi_{1}^{\prime \prime}-i t} \mathscr{D}_{\xi_{1} \xi_{2}} \\
& =\Delta_{\xi_{1}}^{\prime \prime i t} \mathscr{D}_{\xi_{1} \xi_{2}} \\
& =\mathscr{D}_{\xi_{1} \xi_{2}}
\end{aligned}
$$

and

$$
\begin{gathered}
\left(D \omega_{\xi_{1}}^{\prime \prime}: D \omega_{\xi_{2}}^{\prime \prime}\right)_{t} \sigma_{t}^{\xi_{2}}(X)\left(D \omega_{\xi_{1}}^{\prime \prime}: D \omega_{\xi_{2}}^{\prime \prime}\right)_{t}^{*} \xi \\
=\Delta_{\xi_{1} \xi_{2}}^{\prime \prime i t} \Delta_{\xi_{2}}^{\prime \prime}-i t \Delta_{\xi_{2}}^{\prime \prime i t} X \Delta_{\xi_{2}}^{\prime \prime-i t} \Delta_{\xi_{2}}^{\prime \prime i t} \Delta_{\xi_{1}}^{\prime \prime}-i t \xi \\
=\Delta_{\xi_{1} \xi_{2}}^{\prime \prime i t} X \Delta_{\xi_{1} \xi_{2}}^{\prime \prime}-i t \xi \\
=\sigma_{t}^{\xi_{1}}(X) \xi
\end{gathered}
$$

for all $t \in \mathbf{R}, X \in \mathscr{R}\left(\mathscr{M}^{\prime \prime}, \mathscr{D}_{\xi_{1} \xi_{2}}\right)$ and $\xi \in \mathscr{D}_{\xi_{1} \xi_{2}}$. This completes the proof.

By Theorem 3.9 we have the following

Corollary 3.10. Suppose $\left(\mathscr{M}, \mathscr{D}, \xi_{0}\right)$ and $\left(\mathscr{M}, \mathscr{D}, \xi_{1}\right)$ are full standard systems. Then $\left(\xi_{0}, \xi_{1}\right)$ is relative modular for $(\mathscr{M}, \mathscr{D}),\left(D \omega_{\xi_{1}}^{\prime \prime}: D \omega_{\xi_{0}}^{\prime \prime}\right)_{t} / \mathscr{D}$ $\in \mathscr{M}$ for all $t \in \mathbb{R}$ and

$$
\sigma_{t}^{\xi_{1}}(X) \zeta=\left(D \omega_{\xi_{1}}^{\prime \prime}: D \omega_{\xi_{0}}^{\prime \prime}\right)_{t} \sigma_{t}^{\xi_{0}}(X)\left(D \omega_{\xi_{1}}^{\prime \prime}: D \omega_{\xi_{0}}^{\prime \prime}\right)_{t}^{*} \zeta
$$

for all $t \in \mathbf{R}, X \in \mathscr{M}$ and $\zeta \in \mathscr{D}$.

Poposition 3.11. Let $(\mathscr{M}, \mathscr{D})$ be a closed $O_{p}^{*}$-algebra such that $\mathscr{M}^{\prime} \mathscr{D}=\mathscr{D}$ and a pair $\left(\xi_{1}, \xi_{2}\right)$ of vectors in $\mathscr{D}$ be relative modular for $(\mathscr{M}, \mathscr{D})$. Then the following statements are equivalent.

(1) The positive linear functional $\omega_{\xi_{1}}$ on the generalized von Neumann algebra $\mathscr{R}\left(\mathscr{M}^{\prime \prime}, \mathscr{D}_{\xi_{1} \xi_{2}}\right)$ is $\left\{\sigma_{t}^{\xi_{2}}\right\}$-invariant.

(2) The positive linear functional $\omega_{\xi_{2}}$ on $\mathscr{R}\left(\mathscr{M}^{\prime \prime}, \mathscr{D}_{\xi_{1} \xi_{2}}\right)$ is $\left\{\sigma_{t}^{\xi_{1}}\right\}-$ invariant.

(3) $\left\{\left(D \omega_{\xi_{2}}^{\prime \prime}: D \omega_{\xi_{1}}^{\prime \prime}\right)_{t}\right\}_{t \in \mathbf{R}}$ is a strongly continuous one-parameter group of unitary operators in $\mathscr{M}_{\sigma}^{\prime \prime \xi_{1}} \cap \mathscr{M}_{\sigma}^{\prime \xi_{2}}$, where $\mathscr{M}_{\sigma}^{\prime \xi_{i}}$ denotes the fixed-point algebra of $\left\{\sigma_{t}^{\xi_{i}}\right\}$ in $\mathscr{M}^{\prime \prime}(i=1,2)$. 
Proof. (1) $\Rightarrow$ (3) It follows from Theorem 3.9 and the $\left\{\sigma_{t}^{\xi_{2}}\right\}$-invariance of $\omega_{\xi_{1}}$ that

$$
\begin{aligned}
\left(X \xi_{1} \mid \xi_{1}\right) & =\left(\sigma_{t}^{\xi_{2}}(X) \xi_{1} \mid \xi_{1}\right) \\
& \left.=\left(\left(D \omega_{\xi_{2}}^{\prime \prime}: D \omega_{\xi_{1}}^{\prime \prime}\right)\right)_{t}^{\xi_{t}}(X)\left(D \omega_{\xi_{2}}^{\prime \prime}: D \omega_{\xi_{1}}^{\prime \prime}\right)_{t}^{*} \xi_{1} \mid \xi_{1}\right)
\end{aligned}
$$

for each $X \in \mathscr{R}\left(\mathscr{M}^{\prime \prime}, \mathscr{D}_{\xi_{1} \xi_{2}}\right)$ and $t \in \mathbb{R}$, which implies by

$$
\left(D \omega_{\xi_{2}}^{\prime \prime}: D \omega_{\xi_{1}}^{\prime \prime}\right)_{t} / \mathscr{D}_{\xi_{1} \xi_{2}} \in \mathscr{R}\left(\mathscr{M}^{\prime \prime}, \mathscr{D}_{\xi_{1} \xi_{2}}\right)
$$

that

$$
\left(\left(D \omega_{\xi_{2}}^{\prime \prime}: D \omega_{\xi_{1}}^{\prime \prime}\right)_{-t} \xi_{1} \mid X^{\dagger} \xi_{1}\right)=\left(\left(D \omega_{\xi_{2}}^{\prime \prime}: D \omega_{\xi_{1}}^{\prime \prime}\right)_{-t} X \xi_{1} \mid \xi_{1}\right)
$$

for all $X \in \mathscr{M}$ and $t \in \mathbb{R}$ 。 Since $\left(\mathscr{R}\left(\mathscr{M}^{\prime \prime}, \mathscr{D}_{\xi_{1} \xi_{2}}\right), \mathscr{D}_{\xi_{1} \xi_{2}}, \xi_{1}\right)$ is a standard system and $\mathscr{R}\left(\mathscr{M}^{\prime \prime}, \mathscr{D}_{\xi_{1} \xi_{2}}\right) "=\mathscr{M}^{\prime \prime}$ by Lemma 3.7 , it follows from Lemma 3.4 (1) and (3.9) that

$$
\left(\left(D \omega_{\xi_{2}}^{\prime \prime}: D \omega_{\xi_{1}}^{\prime \prime}\right)_{-t} \xi_{1} \mid A^{*} \xi_{1}\right)=\left(\left(D \omega_{\xi_{2}}^{\prime \prime}: D \omega_{\xi_{1}}^{\prime \prime}\right)_{-t} A \xi_{1} \mid \xi_{1}\right)
$$

for all $A \in \mathscr{M}^{\prime \prime}$ and $t \in \mathbb{R}$, which implies the normal form $\omega_{\xi_{1}}^{\prime \prime}$ on $\mathscr{M}^{\prime \prime}$ is $\left\{\sigma_{t}^{\xi_{2}}\right\}$-invariant, so that the statement (3) follows from ([31] Corollary 10.28).

$(3) \Rightarrow(1)$ By ([31] Corollary 10.28) we have

$$
\omega_{\xi_{1}}^{\prime \prime}\left(\sigma_{t}^{\xi_{2}}(A)\right)=\omega_{\xi_{1}}^{\prime \prime}(A)
$$

for all $A \in \mathscr{M}^{\prime \prime}$ and $t \in \mathbb{R}$, which implies

$$
\omega_{\xi_{1}}\left(\sigma_{t}^{\xi_{2}}(X)\right)=\omega_{\xi_{1}}(X)
$$

for all $X \in \mathscr{R}\left(\mathscr{M}^{\prime \prime}, \mathscr{D}_{\xi_{1} \xi_{2}}\right)$ and $t \in \mathbb{R}$.

Similarly, the equivalence of (2) and (3) is shown.

Proposition 3.12. Let ( $\mathscr{M}, \mathscr{D})$ be a closed $O_{p}^{*}$-algebra such that $\mathscr{M}^{\prime} \mathscr{D}=\mathscr{D}$, and a vector $\eta_{0}$ in $\mathscr{D}$ be strongly cyclic for $\mathscr{M}$ and separating for $\mathscr{M}^{\prime \prime}$.

I. Suppose $\eta_{0}$ is tracial; that is,

$$
\left(X Y \eta_{0} \mid \eta_{0}\right)=\left(Y X \eta_{0} \mid \eta_{0}\right)
$$

for each $X, Y \in \mathscr{M}$. Then the following statements hold.

(1) $\eta_{0}$ is a standard vector for $(\mathscr{M}, \mathscr{D})$ with $\Delta_{\eta_{0}}^{\prime \prime}=I$.

(2) Suppose $\xi$ is a modular vector for $(\mathscr{M}, \mathscr{D})$ such that $\eta_{0} \in \mathscr{D}_{\xi^{\circ}}$ 
Then, a pair $\left(\xi, \eta_{0}\right)$ is relative modular for $(\mathscr{M}, \mathscr{D})$ with $\mathscr{D}_{\xi \eta_{0}}=\mathscr{D}_{\xi}$, and $\left\{\left(D \omega_{\xi}^{\prime \prime}: D \omega_{\eta_{0}}^{\prime \prime}\right)_{t}\right\}_{t \in \mathbf{R}}$ is a strongly continuous one-parameter group of unitary operators, which satisfies

$$
\begin{aligned}
& \left(D \omega_{\xi}^{\prime \prime}: D \omega_{\eta_{0}}^{\prime \prime}\right)_{t} \mathscr{D}_{\xi}=\mathscr{D}_{\xi} \text { and } \\
& \sigma_{t}^{\xi}(X) \zeta=\left(D \omega_{\xi}^{\prime \prime}: D \omega_{\eta_{0}}^{\prime \prime}\right)_{t} X\left(D \omega_{\xi}^{\prime \prime}: D \omega_{\eta_{0}}^{\prime \prime}\right)_{t}^{*} \zeta
\end{aligned}
$$

for each $t \in \mathbf{R}, X \in \mathscr{R}\left(\mathscr{M}^{\prime \prime}, \mathscr{D}_{\xi}\right)$ and $\zeta \in \mathscr{D}_{\xi}$.

II. Conversely, suppose there exists a modular vector $\xi_{0}$ for $(\mathscr{M}, \mathscr{D})$ such that $\eta_{0} \in \mathscr{D}_{\xi_{0}},\left(D \omega_{\xi_{0}}^{\prime \prime}: D \omega_{\eta_{0}}^{\prime \prime}\right)_{t} \mathscr{D}_{\xi_{0}}=\mathscr{D}_{\xi_{0}}$ for each $t \in \mathbb{R}$ and

$$
\sigma_{t}^{\xi_{0}}(X) \zeta=\left(D \omega_{\xi_{0}}^{\prime \prime}: D \omega_{\eta_{0}}^{\prime \prime}\right)_{t} X\left(D \omega_{\xi_{0}}^{\prime \prime}: D \omega_{\eta_{0}}^{\prime \prime}\right)_{t}^{*} \zeta
$$

for each $t \in \mathbb{R}, X \in \mathscr{M}$ and $\zeta \in \mathscr{D}_{\xi_{0}}$. Then $\eta_{0}$ is a tracial vector.

Proof. I. (1) Suppose $\eta_{0}$ is a tracial vector. Then it is easily shown that $S_{\eta_{0}}$ equals the isometry $J_{\eta_{0}}$, and hence it follows from $S_{\eta_{0}} \subset S_{\eta_{0}}^{\prime \prime}$ that $S_{\eta_{0}}=S_{\eta_{0}}^{\prime \prime}=J_{\eta_{0}}=J_{\eta_{0}}^{\prime \prime}$. Hence, the statement (1) holds.

(2) Suppose $\xi$ is a modular vector for $(\mathscr{M}, \mathscr{D})$ such that $\eta_{0} \in \mathscr{D}_{\xi}$. By $(1)$, a pair $\left(\xi, \eta_{0}\right)$ is relative modular for $(\mathscr{M}, \mathscr{D})$ with $\mathscr{D}_{\xi \eta_{0}}=\mathscr{D}_{\xi}$, and hence from Proposition $3.10 \quad\left\{\left(D \omega_{\xi}^{\prime \prime}: D \omega_{\eta_{0}}^{\prime \prime}\right)_{t}\right\}_{t \in \boldsymbol{R}}$ is a strongly continuous one-parameter group of unitary operators, and further by Theorem 3.9

$$
\begin{aligned}
\left(D \omega_{\xi}^{\prime \prime}: D \omega_{\eta_{0}}^{\prime \prime}\right)_{t} \mathscr{D}_{\xi}=\mathscr{D}_{\xi}, & \\
\sigma_{t}^{\xi}(X) \zeta & =\left(D \omega_{\xi}^{\prime \prime}: D \omega_{\eta_{0}}^{\prime \prime}\right)_{t} \sigma_{t}^{\eta_{0}}(X)\left(D \omega_{\xi}^{\prime \prime}: D \omega_{\eta_{0}}^{\prime \prime}\right)_{t}^{*} \zeta \\
& =\left(D \omega_{\xi}^{\prime \prime}: D \omega_{\eta_{0}}^{\prime \prime}\right)_{t} X\left(D \omega_{\xi}^{\prime \prime}: D \omega_{\eta_{0}}^{\prime \prime}\right)_{t}^{*} \zeta
\end{aligned}
$$

for each $t \in \mathbb{R}, X \in \mathscr{R}\left(\mathscr{M}^{\prime \prime}, \mathscr{D}_{\xi}\right)$ and $\zeta \in \mathscr{D}_{\xi}$.

II. Since $\left(D \omega_{\xi_{0}}^{\prime \prime}: D \omega_{\eta_{0}}^{\prime \prime}\right)_{t} \mathscr{D}_{\xi_{0}}=\mathscr{D}_{\xi_{0}}$ for each $t \in \mathbb{R}$, we have

$$
\begin{aligned}
\Delta_{\eta_{0}}^{\prime \prime i t} \mathscr{D}_{\xi_{0}} & =\Delta_{\xi_{0}}^{\prime \prime \prime i t}\left(\Delta_{\xi_{0}}^{\prime \prime}-i t \Delta_{\xi_{0} \eta_{0}}^{\prime \prime \prime i t}\right)\left(\Delta_{\xi_{0} \eta_{0}}^{\prime \prime}-i t \Delta_{\eta_{0}}^{\prime \prime i t}\right) \mathscr{D}_{\xi_{0}} \\
& \subset \Delta_{\xi_{0}}^{\prime \prime i t} \mathscr{M}^{\prime} \mathscr{D}_{\xi_{0}} \\
& =\mathscr{D}_{\xi_{0}}
\end{aligned}
$$

for each $t \in \mathbb{R}$, which implies that the pair $\left(\xi_{0}, \eta_{0}\right)$ is relative modular for $(\mathscr{M}, \mathscr{D})$ with $\mathscr{D}_{\xi_{0_{0} \eta_{0}}}=\mathscr{D}_{\xi_{0^{\circ}}}$ It hence follows from Theorem 3.9 that 


$$
\sigma_{t}^{\xi_{0}}(X) \zeta=\left(D \omega_{\xi_{0}}^{\prime \prime}: D \omega_{\eta_{0}}^{\prime \prime}\right)_{t} \sigma_{t}^{\eta_{0}}(X)\left(D \omega_{\xi_{0}}^{\prime \prime}: D \omega_{\eta_{0}}^{\prime \prime}\right)_{t}^{*} \zeta
$$

for all $t \in \mathbb{R}, X \in \mathscr{M}$ and $\zeta \in \mathscr{D}_{\xi_{0}}$, which implies by (3.10)

$$
\sigma_{t}^{\eta_{0}}(X) \zeta=X \zeta
$$

for each $t \in \mathbb{R}, X \in \mathscr{M}$ and $\zeta \in \mathscr{D}_{\xi_{0}}$. Since the positive linear functional $\omega_{\eta_{0}}$ on $\mathscr{R}\left(\mathscr{M}^{\prime \prime}, \mathscr{D}_{\xi_{0}}\right)$ satisfies the KMS-condition with respect to $\left\{\sigma_{t}^{\eta_{0}}\right\}$ by Theorem 3.2, for each $X, Y \in \mathscr{M}$ there exists a function $f_{X, Y}$ in $A(0,1)$ such that

$$
\begin{aligned}
& f_{X, Y}(t)=\omega_{\eta_{0}}\left(\sigma_{t}^{\eta_{0}}(X) Y\right)=\omega_{\eta_{0}}(X Y), \\
& f_{X, Y}(t+i)=\omega_{\eta_{0}}\left(Y \sigma_{t}^{\eta_{0}}(X)\right)=\omega_{\eta_{0}}(Y X)
\end{aligned}
$$

for all $t \in \mathbb{R}$, which implies

$$
\omega_{\eta_{0}}(X Y)=\omega_{\eta_{0}}(Y X)
$$

for each $X, Y \in \mathscr{M}$; that is, $\eta_{0}$ is a tracial vector. This completes the proof.

We give some concrete examples for standard systems and relative modular vectors.

(i) Let $\mathscr{M}_{0}$ be a von Neumann algebra on a Hilbert space $\mathfrak{S}, T$ be a positive self-adjoint unbounded operator in $\mathfrak{S}$ affiliated with $\mathscr{M}_{0}$ and $\mathscr{D}^{\infty}(T)=\bigcap_{n=1}^{\infty} \mathscr{D}\left(T^{n}\right)$. Then the following statements hold.

$$
\begin{gathered}
\mathscr{R}\left(\mathscr{M}_{0}, \mathscr{D}^{\infty}(T)\right)={\overline{\mathscr{M}_{0}^{\mathscr{D}^{\infty}(T)}}}^{t_{s}^{*} \text { in } \mathscr{L}^{\dagger}\left(\mathscr{D}^{\infty}(T)\right)}, \text { where } \\
\mathscr{M}_{0}^{\mathscr{O}^{\infty}(T)}=\left\{A / \mathscr{D}^{\infty}(T) ; A \in \mathscr{M}_{0,} A \mathscr{D}^{\infty}(T) \subset \mathscr{D}^{\infty}(T),\right. \\
\left.A^{*} \mathscr{D}^{\infty}(T) \subset \mathscr{D}^{\infty}(T)\right\}
\end{gathered}
$$

which are self-adjoint generalized von Neumann algebra containing $\left\{T^{n}\right\}_{n \in \mathbb{N}}$ whose induced topology $t_{\mathscr{R}_{\left(\mathscr{M}_{0}, \mathscr{D}^{\infty}(T)\right)}}$ equals the Fréchet topology defined by the seminorms $\left\{\|\bullet\|_{n}=\left\|T^{n} \circ\right\| ; n \in \mathbb{N}\right\}$.

(2) Suppose $\xi_{0}$ is a cyclic and separating vector for $\mathscr{M}_{0}$ and $T$ is affiliated with the fixed-point algebra $\mathscr{M}_{0}^{\sigma^{\xi_{0}}}$ of $\left\{\sigma_{t}^{\xi_{0}}\right\}$ in $\mathscr{M}_{0}$ such that $\xi_{0} \in \mathscr{D}^{\infty}(T)$. Then $\left(\mathscr{R}\left(\mathscr{M}_{0}, \mathscr{D}^{\infty}(T)\right), \mathscr{D}^{\infty}(T), \xi_{0}\right)$ is a full standard system.

(3) Suppose $\xi_{1}$ and $\xi_{2}$ are cyclic and separating vectors for $\mathscr{M}_{0}$ and $T$ is affliated with $\mathscr{M}_{0}^{\sigma_{1}^{\xi_{1}}} \cap \mathscr{M}_{0}^{\sigma \xi_{2}}$ such that $\xi_{1}, \xi_{2} \in \mathscr{D}^{\infty}(T)$. Then $\left(\xi_{1}, \xi_{2}\right)$ is 
relative modular for $\left(\mathscr{R}\left(\mathscr{M}_{0}, \mathscr{D}^{\infty}(T)\right), \mathscr{D}^{\infty}(T)\right)$ with $\mathscr{D}_{\xi_{1} \xi_{2}}=\mathscr{D}^{\infty}(T)$. By Theorem 3. 9, $\left\{\sigma_{t}^{\xi_{1}}\right\}$ and $\left\{\sigma_{t}^{\xi_{2}}\right\}$ are one-parameter groups of $*$-automorphisms of $\mathscr{R}\left(\mathscr{M}_{0}, \mathscr{D}^{\infty}(T)\right),\left(D \omega_{\xi_{1}}^{\prime \prime}: D \omega_{\xi_{2}}^{\prime \prime}\right)_{t} / \mathscr{D}^{\infty}(T) \in \mathscr{R}\left(\mathscr{M}_{0}, \mathscr{D}^{\infty}(T)\right)$ for all $t \in \mathbf{R}$ and

$$
\sigma_{t}^{\xi_{1}}(X) \zeta=\left(D \omega_{\xi_{1}}^{\prime \prime}: D \omega_{\xi_{2}}^{\prime \prime}\right)_{t} \sigma_{t}^{\xi_{2}}(X)\left(D \omega_{\xi_{1}}^{\prime \prime}: D \omega_{\xi_{2}}^{\prime \prime}\right)_{t}^{*} \zeta
$$

for all $t \in \mathbf{R}, X \in \mathscr{R}\left(\mathscr{M}_{0}, \mathscr{D}^{\infty}(T)\right)$ and $\zeta \in \mathscr{D}^{\infty}(T)$.

(ii) Let $\mathscr{S}=\mathscr{S}(\mathbf{R})$ be the Schwartz space of infinitely differentiable rapidly decreasing functions and let

$$
N=\sum_{n=0}^{\infty}(n+1) f_{n} \otimes \overline{f_{n}},
$$

where $\left\{f_{n}\right\}$ is an orthonormal basis in the Hilbert space $L^{2}=L^{2}(\mathbf{R})$ contained in $\mathscr{S}$ consisting of the normalized Hermite functions. Then $\mathscr{S}=\mathscr{D}^{\infty}(N)$, and hence $\mathscr{L}^{\dagger}(\mathscr{S})$ is a selfadjoint $O_{p}^{*}$-algebra containing the inverse $N$ of a positive Hilbert-Schmidt operator, which implies that a self-adjoint representation $\pi$ of $\mathscr{L}^{\dagger}(\mathscr{S})$ on $L^{2} \otimes \overline{L^{2}}$ is defined by

$$
\pi(X) T=X T, \quad T \in \mathscr{S} \otimes \overline{L^{2}},
$$

where $L^{2} \otimes \overline{L^{2}}$ denotes the Hilbert space of Hilbert-Schmidt operators on $L^{2}$ and $\mathscr{S} \otimes L^{2}=\left\{T \in L^{2} \otimes \overline{L^{2}} ; T L^{2} \subset \mathscr{S}\right\}$. We put

$$
\begin{aligned}
& \boldsymbol{s}_{+}=\left\{\left\{\alpha_{n}\right\} ; \alpha_{n}>0 \quad \text { for } \quad n=0,1,2, \ldots\right. \\
& \text { and } \left.\sup _{n} n^{k}\left|\alpha_{n}\right|<\infty \quad \text { for } \text { each } k \in \mathbf{N}\right\}, \\
& \Omega_{\left\{\alpha_{n}\right\}}=\sum_{n=0}^{\infty} \alpha_{n} f_{n} \otimes \overline{f_{n}}, \quad\left\{\alpha_{n}\right\} \in \boldsymbol{s}_{+} .
\end{aligned}
$$

Then the following statements hold. The proofs follow from Section 5 in [14].

(1) $\left(\pi\left(\mathscr{L}^{\dagger}(\mathscr{S})\right), \mathscr{S} \otimes \overline{L^{2}}, \Omega_{\left\{\alpha_{n}\right\}}\right)$ is a full standard system for each $\left\{\alpha_{n}\right\} \in \boldsymbol{s}_{+}$.

(2) Every pair $\left(\Omega_{\left\{\alpha_{n}\right\}}, \Omega_{\left\{\beta_{n}\right\}}\right)$ for $\left\{\alpha_{n}\right\},\left\{\beta_{n}\right\} \in s_{+}$is relative modular for $\left(\pi\left(\mathscr{L}^{\dagger}(\mathscr{S})\right), \mathscr{S} \otimes \overline{L^{2}}\right)$ with $\left(\mathscr{S} \otimes \overline{L^{2}}\right)_{s_{\left.\left(\alpha_{n}\right)^{\prime} \Omega_{n}\right\}}}=\mathscr{S} \otimes \overline{L^{2}}$.

(3) Let $\pi_{1}$ be a self-adjoint representation of the canonical algebra $\mathscr{A}$ for one degree of freedom defined by

$$
\pi_{1}(x)=\pi\left(\pi_{0}(x)\right), x \in \mathscr{A},
$$


where $\pi_{0}$ denotes the Schrödinger representation of $\mathscr{A}_{0}$. Suppose $\left\{\alpha_{n}\right\} \in s_{+}$ satisfies

$$
0<\alpha_{n} \leqq \gamma e^{-n \beta}, \quad n \in \mathbb{N}
$$

for some $\beta>0$ and $\gamma>0$. Then $\Omega_{\left(\alpha_{n}\right)}$ is a standard vector for $\left(\pi_{1}(\mathscr{A})\right.$, $\left.\mathscr{S} \otimes \overline{L^{2}}\right)$ with $\left(\mathscr{S} \otimes \overline{L^{2}}\right)_{\Omega_{\left\{\alpha_{n}\right\}}}=\mathscr{S} \otimes \overline{L^{2}}$.

(4) Suppose $\left\{\alpha_{n}\right\},\left\{\beta_{n}\right\} \in s_{+}$satisfy the condition (3.11). Then $\left(\Omega_{\left(\alpha_{n}\right\},} \Omega_{\left(\beta_{n}\right\}}\right)$ is relative modular for $\left(\pi_{1}(\mathscr{A}), \mathscr{S} \otimes \overline{L^{2}}\right)$ with $\left(\mathscr{S} \otimes \overline{L^{2}}\right)_{\Omega_{\left\{\alpha_{n}\right\}} \Omega_{\left\{\beta_{n}\right\}}}$ $=\mathscr{S} \otimes \overline{L^{2}}$.

\section{§4. Radon-Nikodym Theorems for $\mathbb{O}_{p}^{*}$-algebras}

In this section we study Radon-Nikodym theorems and Lebesquedecomposition theorems for $O_{p}^{*}$-algebras. We first investigate in more detail the Radon-Nikodym theorem and Lebesgue decomposition theorem obtained in [13,16] with the help of Kosaki's results [19] for von Neumann algebras.

Let $(\mathscr{M}, \mathscr{D})$ be a closed $O_{p}^{*}$-algebra such that $\mathscr{M}^{\prime} \mathscr{D}=\mathscr{D}, \xi_{0}$ be a strongly cyclic vector for $\mathscr{M}$ and let $\phi_{0}=\omega_{\xi_{0}}$. For each positive linear functional $\phi$ on $\mathscr{M}$ we put

$$
T_{\phi}^{\phi_{0}} X \xi_{0}=\lambda_{\phi}(X), \quad X \in \mathscr{M} \text { 。 }
$$

In accordance with the Gudder definition [8] and [13], we define the notions of $\phi_{0}$-absolute continuity and $\phi_{0}$-singularity, respectively as follows:

Definition 4. 1. A positive linear functional $\phi$ on $\mathscr{M}$ is said to be $\phi_{0}$-absolutely continuous if $T_{\phi}^{\phi_{0}}$ is a map; and $\phi$ is said to be strongly $\phi_{0}$-absolutely continuous if $T_{\phi}^{\phi_{0}}$ is a closable map of $\mathscr{S}(\mathscr{D})$ into $\mathfrak{S}_{\phi}$; and $\phi$ is said to be $\phi_{0}$-dominated if $T_{\phi}^{\phi_{0}}$ is a continuous map. If for each $X \in \mathscr{M}$ there exists a sequence $\left\{X_{n}\right\}$ in $\mathscr{M}$ such that $\lim _{n \rightarrow \infty} \phi_{0}\left(X_{n}^{\dagger} X_{n}\right)=0$ and $\lim _{n \rightarrow \infty} \phi\left(\left(X_{n}-X\right)^{\dagger}\left(X_{n}-X\right)\right)=0$, then $\phi$ is said to be $\phi_{0}$-singular。

Remark 4.2. (1) The following statements hold immediately.

(a) If $\phi, \phi$ are strongly $\phi_{0}$-absolutely continuous, then so is $\phi+\phi$.

(b) If $0 \leqq \phi \leqq \phi$ and $\phi$ is $\phi_{0}$-singular, then so is $\phi$. 
However, an analogous statement (a) (resp. (b)) for $\phi_{0}$-singularity (resp. strongly $\phi_{0}$-absolutely continuity) does not necessarily hold (Example 6.3).

(2) For normal forms on a von Neumann algebra with a cyclic and separating vector $\xi_{0}$ the notions of $\phi_{0}$-absolute continuity and $\phi_{0}$-singularity defined by Kosaki [19] are identical with the notions of strongly $\phi_{0}$-absolute continuity and $\phi_{0}$-singularity defined the above, respectively.

It is easily shown that bounded linear maps $T_{\phi_{0}}^{\phi_{0}+\phi}$ and $T_{\phi}^{\phi_{0}+\phi}$ defined by

$$
\begin{aligned}
& T_{\phi_{0}}^{\phi_{0}+\phi} \lambda_{\phi_{0}+\phi}(X)=X \xi_{0}, \\
& T_{\phi}^{\phi_{0}+\phi} \lambda_{\phi_{0}+\phi}(X)=\lambda_{\phi}(X), \quad X \in \mathscr{M}
\end{aligned}
$$

satisfy

$$
\begin{aligned}
& \left(T_{\phi_{0}}^{\phi_{0}+\phi}\right) * T_{\phi_{0}}^{\phi_{0}+\phi},\left(T_{\phi}^{\phi_{0}+\phi}\right) * T_{\phi}^{\phi_{0}+\phi} \in \pi_{\phi_{0}+\phi}(\mathscr{M})^{\prime}, \\
& \left(T_{\phi_{0}}^{\phi_{0}+\phi}\right) * T_{\phi_{0}}^{\phi_{0}+\phi}+\left(T_{\phi}^{\phi_{0}+\phi}\right) * T_{\phi}^{\phi_{0}+\phi}=I .
\end{aligned}
$$

Further, we have by $(4.1)$

$$
\begin{aligned}
& \overline{\left\{\left(X \xi_{0}, \lambda_{\phi}(X)\right) ; X \in \mathscr{M}\right\}}=\left\{\left(T_{\phi_{0}}^{\phi_{0}+\phi} \zeta, T_{\phi}^{\phi_{0}+\phi} \zeta\right) ; \zeta \in \mathfrak{S}_{\phi_{0}+\phi}\right\}, \\
& C_{p}\left(T_{\phi}^{\phi_{0}}\right)=\left(\begin{array}{ll}
T_{\phi_{0}}^{\phi_{0}+\phi}\left(T_{\phi_{0}}^{\phi_{0}+\phi}\right) * & T_{\phi_{0}}^{\phi_{0}+\phi}\left(T_{\phi}^{\phi_{0}+\phi}\right)^{*} \\
T_{\phi}^{\phi_{0}+\phi}\left(T_{\phi_{0}}^{\phi_{0}+\phi}\right) * & T_{\phi}^{\phi_{0}+\phi}\left(T_{\phi}^{\phi_{0}+\phi}\right) *
\end{array}\right),
\end{aligned}
$$

where $C_{p}\left(T_{\phi}^{\phi_{0}}\right)$ denotes the projection from $\mathfrak{S}_{\phi_{0}+\phi} \oplus \mathfrak{S}_{\phi_{0}+\phi}$ onto

$$
\overline{\left\{\left(\mathrm{X} \xi_{0}, \lambda_{\phi}(X)\right) ; X \in \mathscr{M}\right\}} \text {. }
$$

Using these facts, in analogous with [19] we can characterize the notions of strongly $\phi_{0}$-absolute continuity and $\phi_{0}$-singularity by the maps $T_{\phi_{0}}^{\phi_{0}+\phi}$ and $T_{\phi}^{\phi_{0}+\phi}$ as follows :

Lemma 4.3. Let $\phi$ be a positive linear functional on $\mathscr{M}$.

I. The following statements are equivalent.

(1) $\phi$ is strongly $\phi_{0}$-absolutely continuous. 
(2) $T_{\phi_{0}}^{\phi_{0}+\phi}$ is non-singular.

In this case, $\mathscr{D}\left(\overline{T_{\phi}^{\phi_{0}}}\right)=\mathscr{R}\left(T_{\phi_{0}}^{\phi_{0}+\phi}\right), \mathscr{R}\left(\overline{T_{\phi}^{\phi_{0}}}\right)=\mathscr{R}\left(T_{\phi}^{\phi_{0}+\phi}\right)$ and

$$
\overline{T_{\phi}^{\phi_{0}}}=T_{\phi}^{\phi_{0}+\phi}\left(T_{\phi_{0}}^{\phi_{0}+\phi}\right)^{-1}
$$

II. The following statements are equivalent.

(1) $\phi$ is $\phi_{0}$-singular。

(2) $T_{\phi_{0}}^{\phi_{0}+\phi}$ is a partial isometry.

(2) ${ }^{\prime} T_{\phi_{0}}^{\phi_{0}+\phi}\left(T_{\phi_{0}}^{\phi_{0}+\phi}\right)^{*}=I_{\text {\$(D) }}$.

(3) $T_{\phi}^{\phi_{0}+\phi}$ is a partial isometry.

(3) ${ }^{\prime} T_{\phi}^{\phi_{0}+\phi}\left(T_{\phi}^{\phi_{0}+\phi}\right)^{*}=I_{\$_{\phi^{\circ}}}$

(4) $\overline{\left\{\left(X \xi_{0}, \lambda_{\phi}(X)\right) ; X \in \mathscr{M}\right\}}=\mathscr{S}(\mathscr{D}) \notin \mathscr{S}_{\phi}$

(5) inf $\left\{\phi_{0}\left(X^{\dagger} X\right)+\phi\left(Y^{\dagger} Y\right) ; X, Y \in \mathscr{M}, X+Y=Z\right\}=0$

for each $Z \in \mathscr{M}$.

$(5)^{\prime}$ inf $\left(\phi_{0}\left(X^{\dagger} X\right)+\phi\left(Y^{\dagger} Y\right) ; X, Y \in \mathscr{M}, X+Y=I\right\}=0$ 。

We denote by $P(\mathscr{M})$ the set of all positive linear functionals on $\mathscr{M}$. Then, by an order relation $\phi \leqq \psi\left(\phi\left(X^{\dagger} X\right) \leqq \phi\left(X^{\dagger} X\right)\right.$ for each $X \in \mathscr{M})(P(\mathscr{M}), \leqq)$ is an ordered set. We donote by $P(\mathscr{M}, \phi)$ the set of all elements $\phi$ of $P(\mathscr{M})$ such that $\phi \leqq \phi$, and denote by $P_{c}^{\phi_{0}}(\mathscr{M}, \phi) \quad\left(\right.$ resp. $\left.P_{s}^{\phi_{0}}(\mathscr{M}, \phi)\right)$ the set of all strongly $\phi_{0}$-absolutely continuous (resp. $\phi_{0}$-singular) elements of $P(\mathscr{M}, \phi)$ 。

Lemma 4.4. Suppose $\phi$ is a positive linear functional on $\mathscr{M}$ such that $\pi_{\phi_{0}+\phi}(\mathscr{M})^{\prime}$ is a von Neumann algebra. Then the following statements hold.

(1) The isometry $U_{\phi}$ of $\mathfrak{S}_{\mathcal{C}}(\mathscr{D})$ into $\mathfrak{S}_{\phi_{0}+\phi}$ defined by

$$
X \xi_{0} \longrightarrow\left(\left(T_{\phi_{0}}^{\phi_{0}+\phi}\right) * T_{\phi_{0}}^{\phi_{0}+\phi}\right)^{1 / 2} \lambda_{\phi_{0}+\phi}(X), \quad X \in \mathscr{M}
$$

satisfies

$$
\begin{aligned}
& U_{\phi}^{*} \mathscr{D}\left(\pi_{\phi_{0}+\phi}\right) \subset \mathscr{D}^{*}(\mathscr{M}) \text { and } X^{*} U_{\phi}^{*} \xi= \\
& U_{\phi}^{*} \pi_{\phi_{0}+\phi}\left(X^{\dagger}\right) \xi \text { for each } X \in \mathscr{M} \text { and } \xi \in \mathscr{D}\left(\pi_{\phi_{0}+\phi}\right) \text {. }
\end{aligned}
$$

(2) A sequence $\left\{H_{n}^{\prime \phi}\right\}$ of positive operators on $\mathfrak{S}(\mathscr{D})$ defined by 


$$
H_{n}^{\prime \phi}=U_{\phi}^{*}\left(\int_{1 / n}^{1} \lambda^{-1}(1-\lambda) d E(\lambda)\right) U_{\phi}, \quad n \in N
$$

satisfies

$$
\left\{H_{n}^{\prime \phi}\right\} \subset \mathscr{M}^{\prime}, H_{1}^{\prime \phi} \leqq H_{2}^{\prime \phi} \leqq \ldots \text { and }
$$

$\lim _{n \rightarrow \infty}\left(H_{n}^{\prime \phi}\right)^{1 / 2} X \xi_{0}$ exists for each $X \in \mathscr{M}$,

where $\int_{0}^{1} \lambda d E(\lambda)$ is the spectral resolution of $\left(T_{\phi_{0}}^{\phi_{0}+\phi}\right) * T_{\phi_{0}}^{\phi_{0}+\phi}$.

(3) Put

$$
\begin{aligned}
& \phi_{c}(X)=\lim _{n \rightarrow \infty}\left(H_{n}^{\prime \phi} X \xi_{0} \mid \xi_{0}\right), \\
& \phi_{s}(X)=\left(P_{\phi_{0}}^{\phi_{0}+\phi} \lambda_{\phi_{0}+\phi}(X) \mid \lambda_{\phi_{0}+\phi}(I)\right), \quad X \in \mathscr{M},
\end{aligned}
$$

where $P_{\phi_{0}}^{\phi_{0}+\phi}$ is the projection from $\mathscr{S}_{\phi_{0}+\phi}$ onto $\operatorname{Ker}\left(T_{\phi_{0}}^{\phi_{0}+\phi}\right) * T_{\phi_{0}}^{\phi_{0}+\phi}$. Then $\phi_{c}, \phi_{s} \in P(\mathscr{M}, \phi)$ and $\phi=\phi_{c}+\phi_{s}$.

Proof. (1) This is easily proved.

(2) Since $\pi_{\phi_{0}+\phi}(\mathscr{M})^{\prime}$ is a von Neumann algebra, it follows that $K_{n} \equiv \int_{1 / n}^{1} \lambda^{-1}(1-\lambda) d E(\lambda) \in \pi_{\phi_{0}+\phi}(\mathscr{M})^{\prime}$ for $n \in \mathbb{N}$, which implies $H_{n}^{\prime \phi} \in \mathscr{M}^{\prime}$ for $n \in \mathbb{N}$. Further, since $U_{\phi} U_{\phi}^{*}\left(\left(T_{\phi_{0}}^{\phi_{0}+\phi}\right) * T_{\phi_{0}}^{\phi_{0}+\phi}\right)^{1 / 2}=\left(\left(T_{\phi_{0}}^{\phi_{0}+\phi}\right) * T_{\phi_{0}}^{\phi_{0}+\phi}\right)^{1 / 2}$, it follows that $\left(H_{n}^{\prime \phi}\right)^{1 / 2}=U_{\phi}^{*} K_{n}^{1 / 2} U_{\phi}$ for $n \in \mathbf{N}$, which implies that $H_{1}^{\prime \phi} \leqq H_{2}^{\prime \phi} \leq \ldots$ and

$$
\begin{aligned}
\lim _{n, m \rightarrow \infty} & \|\left(H_{n}^{\prime \phi}\right)^{1 / 2} X \xi_{0}-\left.\left(H_{m}^{\prime \phi}\right)^{1 / 2} X \xi_{0}\right|^{2} \\
= & \lim _{n, m \rightarrow \infty}\left\{\left(\left(T_{\phi_{0}}^{\phi_{0}+\phi}\right) * T_{\phi_{0}}^{\phi_{0}+\phi} K_{n} \lambda_{\phi_{0}+\phi}(X) \mid \lambda_{\phi_{0}+\phi}(X)\right)\right. \\
& -\left(\left(\left(T_{\phi_{0}}^{\phi_{0}+\phi}\right) * T_{\phi_{0}}^{\phi_{0}+\phi}\right)^{1 / 2} K_{n}^{1 / 2} \lambda_{\phi_{0}+\phi}(X) \mid\left(\left(T_{\phi_{0}}^{\phi_{0}+\phi}\right) * T_{\phi_{0}}^{\phi_{0}+\phi}\right)^{1 / 2} K_{m}^{1 / 2} \lambda_{\phi_{0}+\phi}(X)\right) \\
& -\left(\left(\left(T_{\phi_{0}}^{\phi_{0}+\phi}\right) * T_{\phi_{0}}^{\phi_{0}+\phi}\right)^{1 / 2} K_{m}^{1 / 2} \lambda_{\phi_{0}+\phi}(X) \mid\left(\left(T_{\phi_{0}}^{\phi_{0}+\phi}\right) * T_{\phi_{0}}^{\phi_{0}+\phi}\right)^{1 / 2} K_{n} \lambda_{\phi_{0}+\phi}(X)\right) \\
& \left.+\left(\left(T_{\phi_{0}}^{\phi_{0}+\phi}\right)^{*} T_{\phi_{0}}^{\phi_{0}+\phi} K_{m} \lambda_{\phi_{0}+\phi}(X) \mid \lambda_{\phi_{0}+\phi}(X)\right)\right\} \\
=\lim _{n, m \rightarrow \infty}\left\{\left(\left(T_{\phi}^{\phi_{0}+\phi}\right) * T_{\phi}^{\phi_{0}+\phi}(I-E(1 / n)) \lambda_{\phi_{0}+\phi}(X) \mid \lambda_{\phi_{0}+\phi}(X)\right)\right. & \left.\times(I-E(1 / m)) \lambda_{\phi_{0}+\phi}(X)\right) \\
& -\left(\left(\left(T_{\phi}^{\phi_{0}+\phi}\right)^{*} T_{\phi}^{\phi_{0}+\phi}\right)^{1 / 2}(I-E(1 / n)) \lambda_{\phi_{0}+\phi}(X) \mid\left(\left(T_{\phi}^{\phi_{0}+\phi}\right) * T_{\phi}^{\phi_{0}+\phi}\right)^{1 / 2}\right. \\
& -\left(\left(\left(T_{\phi}^{\phi_{0}+\phi}\right)^{*} T_{\phi}^{\phi_{0}+\phi}\right)^{1 / 2}(I-E(1 / m)) \lambda_{\phi_{0}+\phi}(X) \mid\left(\left(T_{\phi}^{\phi_{0}+\phi}\right)^{*} T_{\phi}^{\phi_{0}+\phi}\right)^{1 / 2}\right. \\
& \left.\times(I-E(1 / n)) \lambda_{\phi_{0}+\phi}(X)\right)
\end{aligned}
$$




$$
\begin{aligned}
& \left.+\left(\left(T_{\phi}^{\phi_{0}+\phi}\right) * T_{\phi}^{\phi_{0}+\phi}(I-E(1 / m)) \lambda_{\phi_{0}+\phi}(X) \mid \lambda_{\phi_{0}+\phi}(X)\right)\right\} \\
= & 0
\end{aligned}
$$

for each $X \in \mathscr{M}$, and hence $\lim _{n \rightarrow \infty}\left(H_{n}^{\prime \phi}\right)^{1 / 2} X \xi_{0}$ exists for each $X \in \mathscr{M}_{\text {。 }}$

(3) This follows from the equality:

$$
\begin{aligned}
\lim _{n \rightarrow \infty}( & \left.H_{n}^{\prime} \phi X \xi_{0} \mid \xi_{0}\right)=\lim _{n \rightarrow \infty}\left(\left(T_{\phi_{0}}^{\phi_{0}+\phi}\right) * T_{\phi_{0}}^{\phi_{0}+\phi} K_{n} \lambda_{\phi_{0}+\phi}(X) \mid \lambda_{\phi_{0}+\phi}(I)\right) \\
= & \lim _{n \rightarrow \infty}\left(\left(T_{\phi}^{\phi_{0}+\phi}\right) * T_{\phi}^{\phi_{0}+\phi}(I-E(I / n)) \lambda_{\phi_{0}+\phi}(X) \mid \lambda_{\phi_{0}+\phi}(I)\right) \\
= & \left(\left(T_{\phi}^{\phi_{0}+\phi}\right) * T_{\phi}^{\phi_{0}+\phi} \lambda_{\phi_{0}+\phi}(X) \mid \lambda_{\phi_{0}+\phi}(I)\right) \\
& -\left(\left(T_{\phi}^{\phi_{0}+\phi}\right)^{*} T_{\phi}^{\phi_{0}+\phi} P_{\phi_{0}}^{\phi_{0}+\phi} \lambda_{\phi_{0}+\phi}(X) \mid \lambda_{\phi_{0}+\phi}(I)\right) \\
= & \phi(X)-\left(\left(I-\left(T_{\phi_{0}}^{\phi_{0}+\phi}\right) * T_{\phi_{0}}^{\phi_{0}+\phi}\right) P_{\phi_{0}}^{\phi_{0}+\phi} \lambda_{\phi_{0}+\phi}(X) \mid \lambda_{\phi_{0}+\phi}(I)\right) \\
= & \phi(X)-\left(P_{\phi_{0}}^{\phi_{0}+\phi} \lambda_{\phi_{0}+\phi}(X) \mid \lambda_{\phi_{0}+\phi}(I)\right) \\
= & \phi(X)-\phi_{\mathrm{s}}(X)
\end{aligned}
$$

for each $X \in \mathscr{M}$. This completes the proof。

By Lemma 4.2, Lemma 4.4 and ([16] Lemma 5.5) we have the following

Theorem 4.5. (Radon-Nikodym theorem) Let $(\mathscr{M}, \mathscr{D})$ be a closed $O_{p}^{*}$-algebra such that $\mathscr{M}^{\prime} \mathscr{D}=\mathscr{D}$ and $\xi_{0}$ be a strongly cyclic vector for $\mathscr{M}_{\text {。 }}$ Suppose $\phi$ is a positive linear functional on $\mathscr{M}$ such that $\pi_{\phi_{0}+\phi}(\mathscr{M})^{\prime}$ is a von Neumann algebra. Then the following statements are equivalent.

(1) $\phi$ is strongly $\phi_{0}$-absolutely continuous.

(2) $T_{\phi_{0}}^{\phi_{0}+\phi}$ is non-singular.

(3) $\phi$ is represented as

$$
\phi(X)=\lim _{n \rightarrow \infty}\left(H_{n}^{\prime} X \xi_{0} \mid \xi_{0}\right), \quad X \in \mathscr{M}
$$

for some sequence $\left\{H_{n}^{\prime}\right\}$ of positive operators in $\mathscr{M}^{\prime}$ such that $H_{1}^{\prime} \leqq H_{2}^{\prime} \leqq \ldots$ and $\lim _{n \rightarrow \infty} H_{n}^{\prime 1 / 2} X \xi_{0}$ exists for each $X \in \mathscr{M}$ 。

(4) $\phi$ is represented as

$$
\phi(X)=\left(X H^{\prime} \xi_{0} \mid H^{\prime} \xi_{0}\right), \quad X \in \mathscr{M}
$$

for some positive self-adjoint operator $H^{\prime}$ affliated with $\mathscr{M}^{\prime}$ such that $\xi_{0} \in \mathscr{D}\left(H^{\prime}\right)$ and $H^{\prime} \xi_{0} \in \mathscr{D}$. 
Theorem 4. 6. (Lebesgue-decomposition theorem) Let $(\mathscr{M}, \mathscr{D})$ be a closed $O_{p}^{*}$-algebra such that $\mathscr{M}^{\prime} \mathscr{D}=\mathscr{D}$ and $\xi_{0}$ be a strongly cyclic vector for $\mathscr{M}$. Suppose $\phi$ is a positive linear functional on $\mathscr{M}$ such that $\pi_{\phi_{0}+\phi}(\mathscr{M})^{\prime}$ is a von Neumann algebra. Then, $\phi_{c}$ is maximal in $P_{c}^{\phi_{0}}(\mathscr{M}, \phi), \phi_{s} \in$ $P_{s}^{\phi_{0}}(\mathscr{M}, \phi)$ and $\phi=\phi_{c}+\phi_{s}$.

Proof. It follows from Lemma 4.4 and Theorem 4.5 that $\phi_{c} \in P_{c}^{\phi_{0}}(\mathscr{M}, \phi)$ and $\phi=\phi_{c}+\phi_{s}$. It is easily shown that $\phi_{s} \in P_{s}^{\phi_{0}}(\mathscr{M}, \phi)$. We show that $\phi_{c}$ is maximal in $P_{c}^{\phi_{0}}(\mathscr{M}, \phi)$. This is proved by analogy with ([19] Theorem 3.3). Take arbitrary $\phi \in P_{c}^{\phi_{0}}(\mathscr{M}, \phi)$. We denote by $T_{\phi_{0}+\psi}^{\phi_{0}+\phi}$ a bounded linear map of $\mathfrak{S}_{\phi_{0}+\phi}$ into $\mathfrak{S}_{\phi_{0}+\psi}$ defined by

$$
\lambda_{\phi_{0}+\phi}(X) \longrightarrow \lambda_{\phi_{0}+\psi}(X) .
$$

Since $\phi$ is strongly $\phi_{0}$-absolutely continuous, it follows from Theorem 4.5 that $T_{\phi_{0}}^{\phi_{0}+\psi}$ is non-singular and $T_{\phi_{0}+\psi}^{\phi_{0}+\phi}=\left(T_{\phi_{0}}^{\phi_{0}+\psi}\right)^{-1} T_{\phi_{0}}^{\phi_{0}+\phi}$. Hence, we have

$$
\begin{aligned}
T_{\phi_{0}+\psi}^{\phi_{0}+\phi} P_{\phi_{0}}^{\phi_{0}+\phi} \lambda_{\phi_{0}+\phi}(X) & =\left(T_{\phi_{0}}^{\phi_{0}+\psi}\right)^{-1} T_{\phi_{0}}^{\phi_{0}+\phi} P_{\phi_{0}}^{\phi_{0}+\phi} \lambda_{\phi_{0}+\phi}(X) \\
& =0
\end{aligned}
$$

for each $X \in \mathscr{M}$, which implies

$$
\begin{aligned}
\phi\left(X^{\dagger} X\right)+\phi_{0}\left(X^{\dagger} X\right) & =\left\|\lambda_{\phi_{0}+\psi}(X)\right\|^{2} \\
& =\left\|T_{\phi_{0}+\phi}^{\phi_{0}+\phi} \lambda_{\phi_{0}+\phi}(X)\right\|^{2} \\
& =\left\|T_{\phi_{0}+\psi}^{\phi_{0}+\phi}\left(I-P_{\phi_{0}}^{\phi_{0}+\phi}\right) \lambda_{\phi_{0}+\phi}(X)\right\|^{2} \\
& \leqq\left\|\left(I-P_{\phi_{0}}^{\phi_{0}+\phi}\right) \lambda_{\phi_{0}+\phi}(X)\right\|^{2} \\
& =\phi_{c}\left(X^{\dagger} X\right)+\phi_{0}\left(X^{\dagger} X\right)
\end{aligned}
$$

for each $X \in \mathscr{M}$. Hence, $\phi \leqq \phi_{c}$. This completes the proof.

Corollary 4.7. I. Suppose $\phi \in P(\mathscr{M})$ satisfies $\pi_{\phi_{0}+\phi}(\mathscr{M})^{\prime}$ is a von Neumann algebra. Then the following statements are equivalent.

(1) $\phi$ is $\phi_{0}-$ singular.

(2) $\phi_{c}=0$.

(3) $P(\mathscr{M}, \phi) \cap P\left(\mathscr{M}, \phi_{0}\right)=\{0\}$. 
II. Suppose $\phi, \phi \in P(\mathscr{M})$ satisfies $\pi_{\phi_{0}+\phi}(\mathscr{M})^{\prime}$ and $\pi_{\phi_{0}+\psi}(\mathscr{M})^{\prime}$ are von Neumann algebras. Then the following statements hold.

(1) $(\lambda \phi)_{c}=\lambda \phi_{c}$ for $\lambda \geqq 0$.

(2) If $0 \leqq \phi \leqq \phi$, then $\phi_{c} \leqq \phi_{c}$.

(3) Further, if $\pi_{\phi_{0}+\phi+\psi}(\mathscr{M})^{\prime}$ is a von Neumann algebra, then $\phi_{c}+\phi_{c}$ $\leqq(\phi+\phi)_{c}$ 。

Proof. I. $\quad(2) \Rightarrow(1)$ This is trivial.

(1) $\Rightarrow(3) \quad$ Take arbitrary $\phi \in P(\mathscr{M}, \phi) \cap P\left(\mathscr{M}, \phi_{0}\right)$. Since $\phi$ is $\phi_{0^{-}}$ singular and $\phi \in P(\mathscr{M}, \phi)$, it follows from Remark 4.2, (a) that $\phi$ is $\phi_{0}$-singular. On the other hand, $\phi$ is strongly $\phi_{0}$-absolutely continuous since $\phi \leqq \phi_{0}$. Hence, $\phi=0$.

(3) $\Rightarrow(2)$ By Theorem 4.5 $\phi_{c}$ is represented as

$$
\phi_{c}(X)=\lim _{n \rightarrow \infty}\left(H_{n}^{\prime \phi} X \xi_{0} \mid \xi_{0}\right), \quad X \in \mathscr{M} \text {. }
$$

Then, it follows that for each $n \in \mathbb{N}$

$$
\lambda \phi_{c}^{n} \in P(\mathscr{M}, \phi) \cap P\left(\mathscr{M}, \phi_{0}\right)=\{0\}
$$

for some $\lambda>0$, where

$$
\phi_{c}^{n}(X)=\left(H_{n}^{\prime \phi} X \xi_{0} \mid \xi_{0}\right), \quad X \in \mathscr{M},
$$

which implies $\phi_{c}=0$.

II. This follows immediately from Theorem 4.6.

Remark 4.8. (1) In $[13,16]$ we have obtained the Lebesguedecomposition theorem: $\phi_{c} \in P_{c}^{\phi_{0}}(\mathscr{M}, \phi), \phi_{s} \in P_{s}^{\phi_{0}}(\mathscr{M}, \phi)$ and $\phi=\phi_{c}+\phi_{s}$ However, it did not know that $\phi_{c}$ is maximal in $P_{c}^{\phi_{0}}(\mathscr{M}, \phi)$. By Theorem 4.6 this fact is true, but there exists a pathological fact that this Lebesgue decomposition is not unique in general (Example $6.3)$.

(2) By Corollary 4.7 the Kosaki definition of $\phi_{0}$-singularity $P(\mathscr{M}, \phi) \cap P\left(\mathscr{M}, \phi_{0}\right)=\{0\}$ is identical with our definition of $\phi_{0}$-singularity in the case $\pi_{\phi_{0}+\phi}(\mathscr{M})^{\prime}$ is a von Neumann algebra.

We have treated with an unbounded generalization of the TomitaTakesaki theory in [14] and Section 3, so that we now generalize the Radon-Nikodym theorem of Pedersen and Takesaki [24] to that for $O_{p}^{*}$-algebra. 
Theorem 4.9. Let $\left(\mathscr{M}, \mathscr{D}, \xi_{0}\right)$ be a standard system. Then the following statements hold.

I. $\phi$ is a $\phi_{0}$-dominated positive linear functional on $\mathscr{M}$ which satisfies the KMS-condition with respect to $\left\{\sigma_{t}^{\xi_{0}}\right\}$ if and only if $\phi$ is represented as

$$
\phi(X)=\left(X H \xi_{0} \mid H \xi_{0}\right), \quad X \in \mathscr{M}
$$

for some positive operator $H$ in $\mathscr{M}^{\prime} \cap \mathscr{M}^{\prime \prime}$.

II. The following statements are equivalent.

(1) $\phi$ is a strongly $\phi_{0}$-absolutely continuous positive linear functional on $\mathscr{M}$ which satisfies the KMS-condition with respect to $\left\{\sigma_{t}^{\xi_{0}}\right\}$ such that $\phi_{0}+\phi$ is standard.

(2) $\phi$ is represented as

$$
\phi(X)=\lim _{n \rightarrow \infty}\left(H_{n} X \xi_{0} \mid \xi_{0}\right), \quad X \in \mathscr{M}
$$

for some sequence $\left\{H_{n}\right\}$ of positive operators in $\mathscr{M}^{\prime} \cap \mathscr{M}^{\prime \prime}$ such that $H_{1} \leqq H_{2} \leqq \ldots$ and $\lim _{n \rightarrow \infty} H_{n}^{1 / 2} X \xi_{0}$ exists for each $X \in \mathscr{M}$.

(3) $\phi$ is represented as

$$
\phi(X)=\left(X H \xi_{0} \mid H \xi_{0}\right), \quad X \in \mathscr{M}
$$

for some positive self-adjoint operator $H$ affliated with $\mathscr{M}^{\prime} \cap \mathscr{M}^{\prime \prime}$ such that $\xi_{0} \in \mathscr{D}(H)$ and $H \xi_{0} \in \mathscr{D}$.

III. Suppose $\phi$ is a positive linear functional on $\mathscr{M}$ which satisfies the $K M S$-condition with respect to $\left\{\sigma_{t}^{\xi_{0}}\right\}$ such that $\phi_{0}+\phi$ is standard. Then, both the maximal strongly $\phi_{0}$-absolutely continuous part $\phi_{c}$ and the $\phi_{0^{-}}$ singular part $\phi_{s}$ of $\phi$ satisfy the KMS-condition with respect to $\left\{\sigma_{t}^{\xi_{0}}\right\}$.

Proof. I. Since $\phi$ is $\phi_{0}$-dominated, there exists a positive operator $H$ in $\mathscr{M}^{\prime}$ such that

$$
\phi(X)=\left(X H \xi_{0} \mid H \xi_{0}\right)
$$

for all $X \in \mathscr{M}$. Put

$$
\phi^{\prime \prime}(A)=\left(A H \xi_{0} \mid H \xi_{0}\right), \quad A \in \mathscr{M}^{\prime \prime} .
$$

Then $\phi^{\prime \prime}$ is a normal form on the von Neumann algebra $\mathscr{M}^{\prime \prime}$ which satisfies the KMS-condition with respect to $\left\{\sigma_{t}^{\xi_{0}}\right\}$. In fact, take arbitrary $A, B \in \mathscr{M}^{\prime \prime}$. Since $S_{\xi_{0}}^{\prime \prime}=S_{\xi_{0}}$, by Lemma 3.4 there exist sequences $\left\{X_{n}\right\}$ and $\left\{Y_{n}\right\}$ in $\mathscr{M}$ such that $\lim _{n \rightarrow \infty} X_{n} \xi_{0}=A \xi_{0}, \lim _{n \rightarrow \infty} X_{n}^{\dagger} \xi_{0}=A^{*} \xi_{0}, \lim _{n \rightarrow \infty} Y_{n} \xi_{0}$ 
$=B \xi_{0}$ and $\lim Y_{n}^{\dagger} \xi_{0}=B^{*} \xi_{0}$. Since $\phi$ satisfies the KMS-condition with respect to $\left\{\sigma_{t}^{n \rightarrow \infty}\right\}$, there exists a sequence $\left\{f_{X_{n^{n}}, Y_{n}}\right\}$ in $A(0,1)$ such that

$$
\begin{aligned}
& f_{X_{n^{\prime}} Y_{n}}(t)=\phi\left(\sigma_{t}^{\xi_{0}}\left(X_{n}\right) Y_{n}\right)=\left(H^{2} Y_{n} \xi_{0} \mid \Delta_{\xi_{0}}^{i t} X_{n}^{\uparrow} \xi_{0}\right), \\
& f_{X_{n}, Y_{n}}(t+i)=\phi\left(Y_{n} \sigma_{t}^{\xi_{0}}\left(X_{n}\right)\right)=\left(H^{2} \Delta_{\xi_{0}}^{i t} X_{n} \xi_{0} \mid Y_{n}^{\dagger} \xi_{0}\right)
\end{aligned}
$$

for all $t \in \mathbb{R}$ and $n \in \mathbb{N}$, which implies that

$$
\begin{aligned}
& \lim _{n \rightarrow \infty} \sup _{t \in R}\left|f_{X_{n^{\prime}, Y_{n}}}(t)-\left(H^{2} B \xi_{0} \mid \Delta_{\xi_{0}}^{i t} A^{*} \xi_{0}\right)\right|=0, \\
& \lim _{n \rightarrow \infty} \sup _{t \in \mathbb{R}}\left|f_{X_{n^{\prime}}, Y_{n}}(t+i)-\left(H^{2} \Delta_{\xi_{0}}^{i t} A \xi_{0} \mid B^{*} \xi_{0}\right)\right|=0 .
\end{aligned}
$$

Hence, there exists a function $f_{A, B}$ in $A(0,1)$ such that

$$
\begin{aligned}
& f_{A, B}(t)=\left(H^{2} B \xi_{0} \mid \Delta_{\xi_{0}}^{i t} A^{*} \xi_{0}\right)=\phi^{\prime \prime}\left(\sigma_{t}^{\xi_{0}}(A) B\right), \\
& f_{A, B}(t+i)=\left(H^{2} \bigcup_{\xi_{0}}^{i t} A \xi_{0} \mid B^{*} \xi_{0}\right)=\phi^{\prime \prime}\left(B \sigma_{t}^{\xi_{0}}(A)\right)
\end{aligned}
$$

for all $t \in \mathbb{R}$, which means that $\phi^{\prime \prime}$ satisfies the $\mathrm{KMS}$-condition with respect to $\left\{\sigma_{t}^{\xi_{0}}\right\}$. It hence follows from ([32] Theorem 15.4) that $H \in \mathscr{M}^{\prime} \cap \mathscr{M}^{\prime \prime}$. The converse follows from Lemma 3.5.

Suppose $\phi$ is a positive linear functional on $\mathscr{M}$ which satisfies the KMS-condition with respect to $\left\{\sigma_{t}^{\xi_{0}}\right\}$ such that $\phi_{0}+\phi$ is standard. Then it follows from Lemma 4.4 that $H_{n}^{\prime \phi} \in \mathscr{M}^{\prime}$ for $n \in \mathbb{N}, H_{1}^{\prime \phi} \leqq H_{2}^{\prime \phi}$ $\leqq \ldots, \lim _{n \rightarrow \infty}\left(H_{n}^{\prime \phi}\right)^{1 / 2} X \xi$ exists for each $X \in \mathscr{M}$ and

$$
\begin{aligned}
& \phi_{c}(X)=\lim _{n \rightarrow \infty}\left(H_{n}^{\prime \phi} X \xi_{0} \mid \xi_{0}\right), \\
& \phi_{s}(X)=\left(P_{\phi_{0}}^{\phi_{0}+\phi} \lambda_{\phi_{0}+\phi}(X) \mid \lambda_{\phi_{0}+\phi}(I)\right), X \in \mathscr{M} .
\end{aligned}
$$

Since $\phi_{0}+\phi$ is standard, it follows from the above I that

$$
\left(T_{\phi_{0}}^{\phi_{0}+\phi}\right) * T_{\phi_{0}}^{\phi_{0}+\phi} \in \pi_{\phi_{0}+\phi}(\mathscr{M})^{\prime} \cap \pi_{\phi_{0}+\phi}(\mathscr{M})^{\prime \prime} \text { 。 }
$$

We show $H_{n}^{\prime \phi} \in \mathscr{M}^{\prime \prime}$ for $n \in \mathbb{N}$. For each $X, Y, Z \in \mathscr{M}$ and $C \in \mathscr{M}^{\prime}$ we have

$$
\begin{aligned}
& \left(U_{\phi} C U_{\phi}^{*}\left(\left(T_{\phi_{0}}^{\phi_{0}+\phi}\right)^{*} T_{\phi_{0}}^{\phi_{0}+\phi}\right)^{1 / 2} \pi_{\phi_{0}+\phi}(X) \lambda_{\phi_{0}+\phi}(Y) \mid \lambda_{\phi_{0}+\phi}(Z)\right) \\
& \quad=\left(C X Y \xi_{0} \mid U_{\phi}^{*} \lambda_{\phi_{0}+\phi}(Z)\right) \\
& \quad=\left(C Y \xi_{0} \mid U_{\phi}^{*} \pi_{\phi_{0}+\phi}\left(X^{\dagger}\right) \lambda_{\phi_{0}+\phi}(Z)\right) \\
& \left.\quad=\left(U_{\phi} C U_{\phi}^{*}\left(T_{\phi_{0}}^{\phi_{0}+\phi}\right)^{*} T_{\phi_{0}}^{\phi_{0}+\phi}\right)^{1 / 2} \lambda_{\phi_{0}+\phi}(Y) \mid \pi_{\phi_{0}+\phi}\left(X^{\dagger}\right) \lambda_{\phi_{0}+\phi}(Z)\right),
\end{aligned}
$$


and hence $U_{\phi} C U_{\phi}^{*}\left(\left(T_{\phi_{0}}^{\phi_{0}+\phi}\right)^{*} T_{\phi_{0}}^{\phi_{0}+\phi}\right)^{1 / 2} \in \pi_{\phi_{0}+\phi}(\mathscr{M})^{\prime}$, which implies

$$
\begin{aligned}
C H_{n}^{\prime \phi} X \xi_{0} & =C U_{\phi}^{*}\left(\int_{1 / n}^{1} \lambda^{-1}(1-\lambda) d E(\lambda)\right) U_{\phi} X \xi_{0} \\
& =U_{\phi}^{*}\left(U_{\phi} C U_{\phi}^{*}\right)\left(\int_{1 / n}^{1} \lambda^{-1}(1-\lambda) d E(\lambda)\right)\left(\left(T_{\phi_{0}}^{\phi_{0}+\phi}\right) * T_{\phi_{0}}^{\phi_{0}+\phi}\right)^{1 / 2} \lambda_{\phi_{0}+\phi}(X) \\
& \left.=U_{\phi}^{*}\left(U_{\phi} C U_{\phi}^{*}\right)\left(\left(T_{\phi_{0}}^{\phi_{0}+\phi}\right) * T_{\phi_{0}}^{\phi_{0}+\phi}\right)^{1 / 2}\left(\int_{1 / n}^{1} \lambda^{-1}(1-\lambda) d E(\lambda)\right) \lambda_{\phi_{0}+\phi}(X)\right) \\
& =U_{\phi}^{*}\left(\int_{1 / n}^{1} \lambda^{-1}(1-\lambda) d E(\lambda)\right)\left(U_{\phi} C U_{\phi}^{*}\left(\left(T_{\phi_{0}}^{\phi_{0}+\phi}\right)^{*} T_{\phi_{0}}^{\phi_{0}+\phi}\right)^{1 / 2}\right) \lambda_{\phi_{0}+\phi}(X) \\
& =H_{n}^{\prime \phi} C X \xi_{0}
\end{aligned}
$$

for each $X \in \mathscr{M}, C \in \mathscr{M}^{\prime}$ and $n \in \mathbb{N}$. Hence, $H_{n}^{\prime \phi} \in \mathscr{M}^{\prime \prime}$ for all $n \in \mathbb{N}$, which implies the implication (1) $\Rightarrow(2)$ in II.

The implication (2) $\Rightarrow(3)$ in II is similar to the proof of $(2) \Rightarrow$ (3) in Theorem 4. 5.

We show the implication (3) $\Rightarrow(1)$ in II. It is clear that $\phi$ is a strongly $\phi_{0}$-absolutely continuous positive linear functional on $\mathscr{M}$. By Lemma 3.5, (1), $\phi$ satisfies the KMS-condition with respect to $\left\{\sigma_{t}^{\xi_{0}}\right\}$ 。 We note that $\left(1+H^{2}\right)^{1 / 2}$ is an invertible positive self-adjoint operator in $\mathscr{S}(\mathscr{D})$ affiliated with $\mathscr{M}^{\prime} \cap \mathscr{M}^{\prime \prime}$ such that $\mathscr{D}\left(\left(1+H^{2}\right)^{1 / 2}\right)=\mathscr{D}(H) \supset$ $\mathscr{M} \xi_{0}$ and $\phi_{0}+\phi=\omega_{\left(1+H^{2}\right)^{2 / 2} \xi_{0}}$. It hence follows from Lemma 3.4 (1) and Lemma 3.5 (2) that $\phi_{0}+\phi$ is standard.

In the above proof we have proved $H_{n}^{\prime \phi} \in \mathscr{M}^{\prime} \cap \mathscr{M}^{\prime \prime}$ for $n \in \mathbb{N}$, and hence the statement III follows from the statement II. This completes the proof.

Let $\left(\mathscr{M}, \mathscr{D}, \xi_{0}\right)$ be a standard system. Then the following questions arise.

Question I. Suppose $\phi$ is a positive linear functional on $\mathscr{M}$ which satisfies the KMS-condition with respect to $\left\{\sigma_{t}^{\varepsilon_{0}}\right\}$. Then, is $\phi$ automatically strongly $\phi_{0}$-absolutely continuous?

In Section 5 we shall state that the above question is affirmative in case that the $O_{D}^{*}$-algebra $(\mathscr{M}, \mathscr{D})$ satisfies the von Neumann density type theorem; that is, $[\mathscr{M}]_{w o}^{\prime \prime}=\left[\mathscr{M}^{t_{s}^{*}}\right.$. 
Question II. Suppose $\phi$ is a positive linear functional on $\mathscr{M}$ which satisfies the KMS-condition with respect to $\left\{\sigma_{t}^{\xi_{0}}\right\}$. Under what conditions is $\phi$ represented as

$$
\phi=\omega_{H \xi_{0}}
$$

for some positive self-adjoint operator $H$ affiliated with $\mathscr{M}^{\prime} \cap \mathscr{M}^{\prime \prime}$ such that $\xi_{0} \in \mathscr{D}(H)$ and $H \xi_{0} \in \mathscr{D}$ ?

We here consider Question II.

1. If $\phi$ is strongly $\phi_{0}$-absolutely continuous and $\phi_{0}+\phi$ is standard, then Question II is affirmative (Theorem 4.9).

However, it seems to be difficult to show directly that $\phi_{0}+\phi$ is standard, and so we consider when Question II is affirmative without the assumption of the standardness of $\phi_{0}+\phi$.

2. Suppose $\phi$ is represented as

$$
\phi=\omega_{\xi}, \xi \in \mathscr{D}
$$

and the normal form $\omega_{\xi}^{\prime \prime}$ on the von Neumann algebra $\mathscr{M}^{\prime \prime}$ satisfies the $K M S$-condition with respect to $\left\{\sigma_{t}^{\xi_{0}}\right\}$. Then Question II is affirmative.

In fact, by ([32] Theorem 15.4) there exists a positive selfadjoint operator $H$ affiliated with $\mathscr{M}^{\prime} \cap \mathscr{M}^{\prime \prime}$ such that $\xi_{0} \in \mathscr{D}(H)$ and

$$
(A \xi \mid \xi)=\left(A H \xi_{0} \mid H \xi_{0}\right)
$$

for all $A \in \mathscr{M}^{\prime \prime}$. Take an arbitrary $X \in \mathscr{M}$. Since $\mathscr{M}^{\prime} \mathscr{D}=\mathscr{D}$, there is a sequence $\left\{X_{n}\right\}$ in $\mathscr{M}^{\prime \prime}$ such that $\lim _{n \rightarrow \infty} X_{n} \zeta=X \zeta$ for each $\zeta \in \mathscr{D}$. Then it follows from (4.6) and $\xi_{0} \in \mathscr{D}(H)$ that $\left\{X_{n} \xi_{0}\right\} \subset \mathscr{D}(H)$, $\lim _{n \rightarrow \infty} X_{n} \xi_{0}=X \xi_{0}$ and $\lim _{n, m \rightarrow \infty}\left\|H X_{n} \xi_{0}-H X_{m} \xi_{0}\right\|=\lim _{n, m \rightarrow \infty}\left\|X_{n} \xi-X_{m} \xi\right\|=0$, and hence $\mathscr{M} \xi_{0} \subset \mathscr{D}(H)$, and so $H \xi_{0} \in \mathscr{D}$ and $X H \xi_{0}=H X \xi_{0}$, which implies $\phi(X)=\left(X H \xi_{0} \mid H \xi_{0}\right)$ for all $X \in \mathscr{M}$.

3. Suppose $\phi$ is strongly $\phi_{0}$-absolutely continuous, $\pi_{\phi_{0}+\phi}(\mathscr{M})^{\prime}$ is a von Neumann algebra and

$$
\phi\left(X^{\dagger} X\right) \leqq \gamma\left\{\phi_{0}\left(X^{\dagger} X\right)+\phi_{0}\left(X X^{\dagger}\right)\right\}, X \in \mathscr{M}
$$

for some constant $\gamma>0$. Then Question II is affirmative.

In fact, by Theorem $4.5 \phi$ is represented as

$$
\phi=\omega_{H^{\prime} \xi_{0}}
$$

for some positive self-adjoint operator $H^{\prime}$ affiliated $\mathscr{M}^{\prime}$ such that 
$\xi_{0} \in \mathscr{D}\left(H^{\prime}\right)$ and $H^{\prime} \xi_{0} \in \mathscr{D}$. Since $\omega_{H^{\prime} \xi_{0}}$ is $\left\{\sigma_{t}^{\xi_{0}}\right\}$-invariant, we have

$$
\left(H^{\prime} \triangle_{\xi_{0}}^{i t} X \xi_{0} \mid H^{\prime} \Delta_{\xi_{0}}^{i t} Y \xi_{0}\right)=\left(H^{\prime} X \xi_{0} \mid H^{\prime} Y \xi_{0}\right)
$$

for all $X, Y \in \mathscr{M}$. Take arbitrary $A \in \mathscr{M}^{\prime \prime}$. Since $S_{\xi_{0}}^{\prime \prime}=S_{\xi_{0}}$, there exists a sequence $\left\{X_{n}\right\}$ in $\mathscr{M}$ such that $\lim _{n \rightarrow \infty} X_{n} \xi_{0}=A \xi_{0}$ and $\lim _{n \rightarrow \infty} X_{n}^{\dagger} \xi_{0}=A^{*} \xi_{0}$. By (4.7) and (4.8) we have

$$
\lim _{n \rightarrow \infty} H^{\prime} X_{n} \xi_{0}=H^{\prime} A \xi_{0} \text { and } \lim _{n \rightarrow \infty} H^{\prime} \Delta_{\xi_{0}}^{i t} X_{n} \xi_{0}=H^{\prime} \Delta_{\xi_{0}}^{i t} A \xi_{0} .
$$

By (4.8) and (4.9) we have

$$
\left\|H^{\prime} \triangle_{\xi_{0}}^{i t} A \xi_{0}\right\|=\left\|H^{\prime} A \xi_{0}\right\|, \quad\left(H^{\prime} \triangle_{\xi_{0}}^{i t} X \xi_{0} \mid H^{\prime} \Delta_{\xi_{0}}^{i t} A \xi_{0}\right)=\left(H^{\prime} X \xi_{0} \mid H^{\prime} A \xi_{0}\right)
$$

for all $X \in \mathscr{M}$ and $A \in \mathscr{M}^{\prime \prime}$, which implies

$$
\left\|H^{\prime} \triangle_{\xi_{0}}^{i t} X \xi_{0}-H^{\prime} \triangle_{\xi_{0}}^{i t} A \xi_{0}\right\|=\left\|H^{\prime} X \xi_{0}-H^{\prime} A \xi_{0}\right\|
$$

for all $X \in \mathscr{M}$ and $A \in \mathscr{M}^{\prime \prime}$. Since $\omega_{H^{\prime} \xi_{0}}$ satisfies the KMS-condition with respect to $\left\{\sigma_{t}^{\xi_{0}}\right\}$, it follows from (4.9) and (4.10) that the normal form $\omega_{H^{\prime} \xi_{0}}^{\prime \prime}$ on $\mathscr{M}^{\prime \prime}$ satisfies the KMS-condition with respect to $\left\{\sigma_{t}^{\xi_{0}}\right\}$. By the above statement 2 Question II is affirmative.

4. Suppose $\phi$ is represented as

$$
\phi=\omega_{H^{\prime} \xi_{0}}
$$

for some positive self-adjoint operator $H^{\prime}$ affiliated with $\mathscr{M}^{\prime}$ such that $\xi_{0} \in \mathscr{D}\left(H^{\prime 2}\right)$ and $H^{\prime 2} \xi_{0} \in \mathscr{D}$. Then Question II is affirmative.

In fact, since $\mathscr{M} \xi_{0} \subset \mathscr{D}\left(H^{\prime 2}\right), H^{\prime 2} X \xi_{0}=X H^{\prime 2} \xi_{0}$ for each $X \in \mathscr{M}$ and $\omega_{H^{\prime} \xi_{0}}$ is $\left\{\sigma_{t}^{\xi_{0}}\right\}$-invariant, it follows that

$$
H^{2} \Delta_{\xi_{0}}^{i t} X \xi_{0}=\Delta_{\xi_{0}}^{i t} H^{\prime 2} X \xi_{0}
$$

for all $X \in \mathscr{M} \cup \mathscr{M}^{\prime \prime}$ and $t \in \mathbb{R}$, which implies by $S_{\xi_{0}}^{\prime \prime}=S_{\xi_{0}}$ that the normal form $\omega_{H^{\prime} \xi_{0}}^{\prime \prime}$ on $\mathscr{M}^{\prime \prime}$ satisfies the KMS-condition with respect to $\left\{\sigma_{t}^{\xi_{0}}\right\}$. By the statement 2 Question II is affirmative.

5. Suppose $\left(\mathscr{M}, \mathscr{D}, \xi_{0}\right)$ is a full standard system and $\phi$ is strongly $\phi_{0}$-absolutely continuous, $\pi_{\phi_{0}+\phi}(\mathscr{M})^{\prime}$ is a von Neumann algebra and

$$
\phi\left(X^{\dagger} X\right) \leqq \sum_{k=1}^{n} \phi_{0}\left(X^{\dagger} Y_{k} Y_{k} X\right), X \in \mathscr{M}
$$

for some finite subset $\left\{Y_{1}, Y_{2}, \ldots, Y_{n}\right\}$ of $\mathscr{M}$. Then Question $I I$ is affirmative.

In fact, $\phi$ is represented as 


$$
\phi=\omega_{H^{\prime} \xi_{0}}
$$

for some positive self-adjoint operator $H^{\prime}$ affiliated with $\mathscr{M}^{\prime}$ such that $\xi_{0} \in \mathscr{D}\left(H^{\prime}\right)$ and $H^{\prime} \xi_{0} \in \mathscr{D}$. Since $\xi_{0}$ is a strongly cyclic vector for $\mathscr{M}$, it follows from (4.12) that $\mathscr{D} \subset \mathscr{D}\left(H^{\prime}\right)$, which implies $\mathscr{M} \xi_{0}$ $\subset \mathscr{D}\left(H^{\prime 2}\right)$. Hence, by the statement 4 , Question II is affirmative.

6. Suppose the $O_{p}^{*}$-algebra $(\mathscr{M}, \mathscr{D})$ satisfies the von Neumann density type theorem and $\phi$ is $\sigma$-weakly continuous. Then Question II is affirmative (Theorem 5.6).

We study a Radon-Nikodym theorem for $\left\{\sigma_{t}^{\xi_{0}}\right\}$-invariant positive

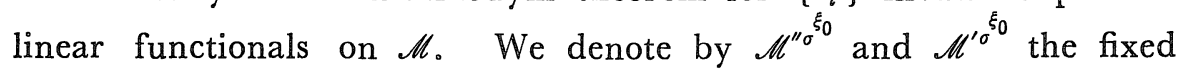
point algebras of $\left\{\sigma_{t}^{\xi_{0}}\right\}$ in $\mathscr{M}^{\prime \prime}$ and $\mathscr{M}^{\prime}$, respectively.

Theorem 4. 10. Let $\left(\mathscr{M}, \mathscr{D}, \xi_{0}\right)$ be a standard system.

I. The following statements are equivalent.

(1) $\phi$ is a $\omega_{\xi_{0}}$-dominated, $\left\{\sigma_{t}^{\xi_{0}}\right\}$-invariant positive linear functional on $\mathscr{M}$.

(2) $\phi$ is represented as

$$
\phi=\omega_{H^{\prime} \xi_{0}}
$$

for some positive operator $H^{\prime}$ in $\mathscr{M}^{\prime \sigma_{0}^{\xi_{0}}}$.

(3) $\phi$ is represented as

$$
\phi=\omega_{H \xi_{0}}
$$

for some positive operator $H$ in $\mathscr{M}^{\prime \prime o^{\xi_{0}}}$ such that $H \xi_{0} \in \mathscr{D}$.

In the following $I I$ and $I I I$, suppose $\phi$ is a positive linear functional on $\mathscr{M}$ such that $\phi_{0}+\phi \leqq \tau$ for some standard positive linear functional $\tau$ on $\mathscr{M}$ which satisfies the KMS-condition with respect to $\left\{\sigma_{t}^{\xi_{0}}\right\}$.

II. Suppose $\phi$ is $\left\{\sigma_{t}^{\xi_{0}}\right\}$-invariant. Then $\phi$ is decomposed into the sum:

$$
\phi=\phi_{c}^{\sigma}+\phi_{s}^{\sigma}
$$

where $\phi_{c}^{\sigma}$ is a strongly $\phi_{0}$-absolutely continuous $\left\{\sigma_{t}^{\xi_{0}}\right\}$-invariant positive linear functional on $\mathscr{M}$ and $\phi_{s}^{\sigma}$ is a $\phi_{0}$-singular, $\left\{\sigma_{t}^{\xi_{0}}\right\}$-invariant positive linear

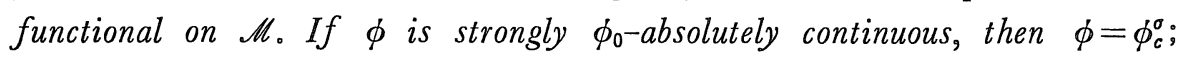
and if $\phi$ is $\phi_{0}$-singular, then $\phi=\phi_{s}^{\sigma}$.

III. The following statements are equivalent. 
(1) $\phi$ is strongly $\phi_{0}$-absolutely continuous and $\left\{\sigma_{t}^{\xi_{0}}\right\}$-invariant.

(2) $\phi$ is represented as

$$
\phi=\omega_{H^{\prime} \xi_{0}}
$$

for some positive self-adjoint operator $H^{\prime}$ affiliated with $\mathscr{M}^{\text {'s }^{\xi_{0}}}$ such that $\xi_{0} \in \mathscr{D}\left(H^{\prime}\right)$ and $H^{\prime} \xi_{0} \in \mathscr{D}$.

(3) $\phi$ is represented as

$$
\phi=\omega_{H \xi_{0}}
$$

for some positive self-adjoint operator $H$ affliated with $\mathscr{M}^{\prime 0^{\xi_{0}}}$ such that $\xi_{0} \in \mathscr{D}(H)$ and $H \xi_{0} \in \mathscr{D}$.

Proof. I. $(1) \Leftrightarrow(2)$ This is trivial.

(2) $\Rightarrow(3) \quad$ Put

$$
H=J_{\xi_{0}} H^{\prime} J_{\xi_{0}}
$$

Then $H$ is a positive operator in $\mathscr{M}^{\prime \prime \sigma_{0}}$ satisfying $H \xi_{0}=H^{\prime} \xi_{0}$, and hence $H \xi_{0} \in \mathscr{D}$ and $\phi=\omega_{H \xi_{0}}$.

$(3) \Rightarrow(2) \quad$ This is similar to the proof of $(2) \Rightarrow(3)$.

II. Since $\tau$ satisfies the $\mathrm{KMS}$-condition with respect to $\left\{\sigma_{t}^{\xi_{0}}\right\}$, it follows from ([14] Lemma 3. 8) that

$$
\Delta_{\tau}^{i t} \lambda_{\tau}(X)=\lambda_{\tau}\left(\sigma_{t}^{\xi_{0}}(X)\right)
$$

for all $X \in \mathscr{M}$ and $t \in \mathbb{R}$. Since $\phi_{0} \leqq \tau$ and $\phi \leqq \tau$, there exist $R, K \in$ $\pi_{\tau}(\mathscr{M})^{\prime}$ such that $0 \leqq R, K \leqq 1$ and

$$
\phi_{0}(X)=\left(R \lambda_{\tau}(X) \mid \lambda_{\tau}(I)\right), \phi(X)=\left(K \lambda_{\tau}(X) \mid \lambda_{\tau}(I)\right)
$$

for $X \in \mathscr{M}$. Using (4.13) and the standardness of $\tau$, we can prove in the same way as in Theorem 4.9 that the normal form on $\pi_{\tau}(\mathscr{M})^{\prime \prime}$ :

$$
A \rightarrow\left(R A \lambda_{\tau}(I) \mid \lambda_{\tau}(I)\right)
$$

satisfies the $\mathrm{KMS}$-condition with respect to $\left\{\sigma_{t}^{\tau}\right\}$ and $A \rightarrow\left(K A \lambda_{\tau}(I) \mid\right.$ $\left.\lambda_{\tau}(I)\right)$ is $\left\{\sigma_{t}^{\tau}\right\}$-invariant. Hence, $R \in \pi_{\tau}(\mathscr{M})^{\prime} \cap \pi_{\tau}(\mathscr{M})^{\prime \prime}$ and $K \in \pi_{\tau}(\mathscr{M})^{\prime \sigma_{\tau}(\mathrm{I})}$. We donote by $U$ the isometry of $\mathfrak{S}(\mathscr{D})$ into $\mathfrak{S}_{\tau}$ defined by:

$$
U X \xi_{0}=R^{1 / 2} \lambda_{\tau}(X), X \in \mathscr{M} \text {. }
$$

We now put 


$$
H_{n}^{\prime}=U^{*}\left(\int_{1 / n}^{1} 1 / \lambda d E(\lambda)\right) K U, n \in \mathbb{N},
$$

where $R=\int_{0}^{1} \lambda d E(\lambda)$ denotes the spectral resolutions of $R$. Since $R$ and $K$ commute, it follows that $\left\{H_{n}^{\prime}\right\}$ is a sequence of positive

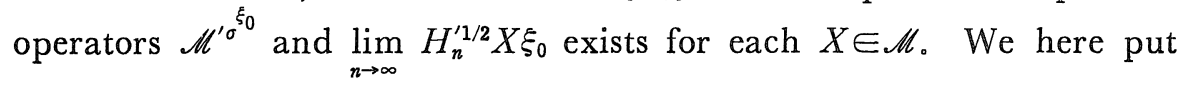

$$
\begin{aligned}
& \phi_{c}^{\sigma}(X)=\lim _{n \rightarrow \infty}\left(H_{n}^{\prime} X \xi_{0} \mid \xi_{0}\right), \\
& \phi_{s}^{\sigma}(X)=\left(K E(0) \lambda_{\tau}(X) \mid \lambda_{\tau}(I)\right), X \in \mathscr{M} .
\end{aligned}
$$

Then it is easily shown that $\phi_{c}^{\sigma}$ is a strongly $\phi_{0}$-absolutely continuous, $\left\{\sigma_{i}^{\xi_{0}}\right\}$-invariant positive linear functional on $\mathscr{M}, \phi_{s}^{\circ}$ is a $\phi_{0}$-singular, $\left\{\sigma_{t}^{\xi_{0}}\right\}$-invariant positive linear functional on $\mathscr{M}$ and $\phi=\phi_{c}^{\sigma}+\phi_{s^{\circ}}^{\sigma}$ Suppose $\phi$ is strongly $\phi_{0}$-absolutely continuous. For each $X \in \mathscr{M}$ there is a sequence $\left\{X_{n}\right\}$ in $\mathscr{M}$ such that $\lim _{n \rightarrow \infty} \lambda_{\tau}\left(X_{n}\right)=E(0) \lambda_{\tau}(X)$.

Then we have

$$
\begin{aligned}
& \lim _{n \rightarrow \infty} X_{n} \xi_{0}=\lim _{n \rightarrow \infty} U R^{1 / 2} X_{n} \xi_{0}=U R^{1 / 2} E(0) \lambda_{\tau}(X)=0, \\
& \lim _{n \rightarrow \infty}\left(\lambda_{\phi}\left(X_{n}\right) \mid \lambda_{\phi}(Y)\right)=\lim _{n \rightarrow \infty}\left(K \lambda_{\tau}\left(X_{n}\right) \mid \lambda_{\tau}(Y)\right) \\
& =\left(K E(0) \lambda_{\tau}(X) \mid \lambda_{\tau}(Y)\right)
\end{aligned}
$$

for each $Y \in \mathscr{M}$. Since $\phi \leqq \tau$ and $\phi$ is strongly $\phi_{0}$-absolutely continuous, we have $\lim _{n \rightarrow \infty} \lambda_{\phi}\left(X_{n}\right)=0$, and hence $\left(K E(0) \lambda_{\tau}(X) \mid \lambda_{\tau}(Y)\right)=0$ for each $Y \in \mathscr{M}$. Hence, $K E(0) \lambda_{\tau}(X)=0$; that is, $\phi_{s}^{\sigma}=0$. Similarly, if $\phi$ is $\phi_{0}$-singular, then $\phi=\phi_{s}^{\sigma}$.

III. $(1) \Leftrightarrow(2)$ Using II, this is proved in similar to the proof of Theorem 4.9.

(2) $\Rightarrow(3) \quad$ Put

$$
H=J_{\xi_{0}} H^{\prime} J_{\xi_{0}}
$$

Then $H$ is a positive self-adjoint operator affiliated with $\mathscr{M}^{\prime \prime \sigma_{0}}$ such that $\xi_{0} \in \mathscr{D}(H), H \xi_{0}=H^{\prime} \xi_{0} \in \mathscr{D}$ and $\phi=\omega_{H \xi_{0}}$.

(3) $\Rightarrow(2) \quad$ This is similar to the proof of $(2) \Rightarrow(3)$. This completes the proof.

Remark. We don't know whether $\phi_{c}^{\sigma}$ is maximal in the subset of $P_{c}^{\phi_{0}}(\mathscr{M}, \phi)$ of $\left\{\sigma_{t}^{\xi_{0}}\right\}$-invariant positive linear functionals or not. 


\section{§5. Radon-Nikodym Theorems for $O_{p}^{*}$-algebras Satisfying the von Neumann Density Type Theorem}

Throughout this section, let $(\mathscr{M}, \mathscr{D})$ be a closed $O_{p}^{*}$-algebra such that $\mathscr{M}^{\prime} \mathscr{D}=\mathscr{D}$ and $[\mathscr{M}]_{w o}^{\prime \prime}=\left[\mathscr{M}^{t_{s}^{*}}\right.$, and $\xi_{0}$ be a cyclic and separating vector for $\mathscr{M}^{\prime \prime}$. We denote by $\mathscr{M}_{*}^{+}$the set of all positive linear functionals which are continuous relative to the $\sigma$-weak topology for $\mathscr{M}$, and denote by $\mathscr{P} \xi_{\xi_{0}}^{\sharp}$ the natural positive cone associated with $\left(\mathscr{M}^{\prime \prime}, \xi_{0}\right)[1,4,11]$.

Theorem 5.1. Suppose $\phi \in \mathscr{M}_{*}^{+}$. Then there exists a unique vector $\xi_{\phi}$ in $\mathscr{P}_{\xi_{0}}^{\sharp} \cap \mathscr{D}$ such that

$$
\phi(X)=\left(X \xi_{\phi} \mid \xi_{\phi}\right)
$$

for all $X \in \mathscr{M}$.

Proof. By ([16] Lemma 5.2) there exists a vector $\xi$ in $\mathscr{D}$ such that $\phi=\omega_{\xi}$. It hence follows from ([31] Theorem 10.25) that

$$
(A \xi \mid \xi)=\left(A \xi_{\phi} \mid \xi_{\phi}\right), A \in \mathscr{M}^{\prime \prime}
$$

for a unique vector $\xi_{\phi}$ in $\mathscr{P}_{\xi_{0}}^{\natural}$. Take an arbitrary $X \in \mathscr{M}$. Let $\left(X^{*} \bar{X}\right)^{1 / 2}$ $=\int_{0}^{\infty} \lambda d E(\lambda)$ be the spectral resolution of $\left(X^{*} \bar{X}\right)^{1 / 2}$, and let $E_{n}=\int_{0}^{n} d E(\lambda)$ for $n \in \mathbf{N}$. Since $\mathscr{M}^{\prime} \mathscr{D}=\mathscr{D}$, it follows that $E_{n}, \bar{X} E_{n} \in \mathscr{M}^{\prime \prime}$ for $n \in \mathbf{N}$. Hence, we have by (5.1)

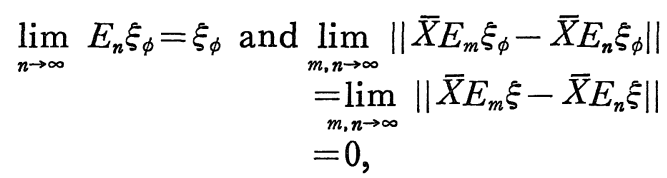

which implies $\xi_{\phi} \in \underset{X \in \mathscr{M}}{\bigcap} \mathscr{D}(\bar{X})=\mathscr{D}$ and $\phi=\omega_{\xi_{\phi^{*}}}$ Suppose $\phi=\omega_{\xi_{1}}=\omega_{\xi_{2}}$ for $\xi_{1}, \xi_{2} \in \mathscr{P} \xi_{0} \cap \mathscr{D}$. Since $\left.[\mathscr{M}]_{w \sigma}^{\prime \prime}=\overline{[\mathscr{M}}\right]^{t_{s}^{*}}$ and (5.1), we have $\xi_{1}=\xi_{2}$ 。 This completes the proof.

Theorem 5.2. Suppose $\phi \in \mathscr{M}_{*}^{+}$. Then the following statements hold.

(1) $\phi$ is strongly $\phi_{0}$-absolutely continuous if and only if $\phi$ is represented as

$$
\phi(X)=\left(X H^{\prime} \xi_{0} \mid H^{\prime} \xi_{0}\right), X \in \mathscr{M}
$$


for some positive self-adjoint operator $H^{\prime}$ affliated with $\mathscr{M}^{\prime}$ such that $\mathscr{M} \xi_{0}$ is a core for $H^{\prime}$. In this case, such an operator $H^{\prime}$ for $\phi$ is unique, which is denoted by $H_{\phi}^{\prime}$.

(2) $\phi$ is $\phi_{0}$-singular if and only if $P(\mathscr{M}, \phi) \cap P\left(\mathscr{M}, \phi_{0}\right)=\{0\}$ 。

(3) $\phi$ is decomposed into the sum:

$$
\phi=\phi_{c}+\phi_{s}
$$

where $\phi_{c}$ is maximal in $P_{c}^{\phi_{0}}(\mathscr{M}, \phi)$ and $\phi_{s} \in P_{s}^{\phi_{0}}(\mathscr{M}, \phi)$.

Proof. By Theorem 5. 1, $\phi_{0}+\phi$ is represented as

$$
\left(\phi_{0}+\phi\right)(X)=\left(X \xi_{\phi_{0}+\phi} \mid \xi_{\phi_{0}+\phi}\right), X \in \mathscr{M}
$$

for a unique vector $\xi_{\phi_{0}+\phi} \in \mathscr{P}_{\xi_{0}}^{\natural} \cap \mathscr{D}$, which implies by $[\mathscr{M}]_{w o}^{\prime \prime}=\overline{[\mathscr{M}}^{t_{s}^{*}}$ that $\xi_{\phi_{0}+\phi}$ is a separating vector for $\mathscr{M}^{\prime \prime}$. Since $\xi_{\phi_{0}+\phi} \in \mathscr{P}_{\xi_{0}}^{\natural}$ it follows that $\xi_{\phi_{0}+\phi}$ is also cyclic for $\mathscr{M}^{\prime \prime}$. We put

$$
U \lambda_{\phi_{0}+\phi}(X)=X \xi_{\phi_{0}+\phi}, X \in \mathscr{M} \text {. }
$$

By (5.2) $U$ is extended to a unitary operator of $\mathfrak{S}_{\phi_{0}+\phi}$ onto $\mathfrak{S}(\mathscr{D})$, which is also denoted by $U$. Using $[\mathscr{M}]_{w \sigma}^{\prime \prime}=\overline{\left[\mathscr{M}^{t}\right]^{*}}$ and $\xi_{\phi_{0}+\phi}$ is a cyclic vector for $\mathscr{M}^{\prime \prime}$, we can prove that $\pi_{\phi_{0}+\phi}(\mathscr{M})^{\prime}=U^{*} \mathscr{M}^{\prime} U$, so that $\pi_{\phi_{0}+\phi}(\mathscr{M})^{\prime}$ is a von Neumann algebra. Hence, the statements (2) and (3) follow from Corollary 4.7 and Theorem 4.6, respectively。

We show the statement (1). Suppose $\phi$ is strongly $\phi_{0}$-absolutely continuous. We denote by $T_{\phi}^{\phi_{0}}$ the closure of a closable map:

$$
X \xi_{0} \in \mathscr{M} \xi_{0} \rightarrow X \xi_{\phi} \in \mathscr{M} \xi_{\phi}
$$

Then, it follows from $[\mathscr{M}]_{w \sigma}^{\prime \prime}=\overline{[\mathscr{M}]^{t_{s}^{*}}}$ that $\mathscr{M}^{\prime \prime} \xi_{0} \subset \mathscr{D}\left(T_{\phi}^{\phi_{0}}\right)$ and $T_{\phi}^{\phi_{0}} A \xi_{0}$ $=A \xi_{\phi}$ for all $A \in \mathscr{M}^{\prime \prime}$, which implies $\mathscr{M}^{\prime \prime} \xi_{0}$ is a core of $T_{\phi}^{\phi_{0}}$ and $T_{\phi}^{\phi_{0}}$ is affiliated with $\mathscr{M}^{\prime}$. Put

$$
H_{\phi}^{\prime}=\left(\left(T_{\phi}^{\phi_{0}}\right) * T_{\phi}^{\phi_{0}}\right)^{1 / 2} \text { 。 }
$$

Then it is easily shown that $H_{\phi}^{\prime}$ is a positive self-adjoint operator affiliated with $\mathscr{M}^{\prime}$ such that $\mathscr{M} \xi_{0}$ is a core for $H_{\phi}^{\prime}$ and $\phi=\omega_{H_{\phi}^{\prime} \xi_{0}^{\circ}}$ The uniqueness of $H_{\phi}^{\prime}$ follows from that of polar decomposition. The converse follows from Theorem 4.5. This completes the proof

Remark 5.3. Representing operators $H^{\prime}$ for $\phi$ in Theorem 4.6 satisfy $\mathscr{M} \xi_{0} \subset \mathscr{D}\left(H^{\prime}\right)$ but without the condition $[\mathscr{M}]_{w \sigma}^{\prime \prime}=\overline{[\mathscr{M}}^{t_{s}^{*}}$, there 
does not necessarily exist a representing operator $H^{\prime}$ for $\phi$ such that $\mathscr{M} \xi_{0}$ is core for $H^{\prime}$.

Remark 5.4. Suppose $\mathscr{M}^{\prime \prime}$ is finite. Then every $\phi \in \mathscr{M}_{*}^{+}$is strongly $\phi_{0}$-absolutely continuous. This is proved in similar to ([19] Corollary 2.3).

Theorem 5.5. Suppose $\left(\mathscr{M}, \mathscr{D}, \xi_{0}\right)$ is a standard system and $\phi \in \mathscr{M}_{*}^{+}$ satisfies the KMS-condition with respect to $\left\{\sigma_{t}^{\xi_{0}}\right\}$. Then the following statements hold.

(1) $\phi$ is represented as

$$
\phi(X)=\left(X H \xi_{0} \mid H \xi_{0}\right), X \in \mathscr{M}
$$

for some positive self-adjoint operator $H$ affiliated with $\mathscr{M}^{\prime} \cap \mathscr{M}^{\prime \prime}$ such that $\xi_{0} \in \mathscr{D}(H)$ and $H \xi_{0} \in \mathscr{D}$. Further, if $\phi$ is faithful; that is, $\phi\left(X^{\dagger} X\right)=0$ implies $X=0$, then $\phi$ is a standard positive linear functional on $\mathscr{M}$ with $\mathscr{D}_{\phi}=\mathscr{D}\left(\pi_{\phi}\right)$.

(2) Suppose $\left(\mathscr{M}, \mathscr{D}, \xi_{0}\right)$ is full. Then $\phi$ is a standard positive linear functional on $\mathscr{M}$ with $\mathscr{D}_{\phi}=\mathscr{D}\left(\pi_{\phi}\right)$. Further, if $\phi$ is faithful, then $\left(\pi_{\phi}(\mathscr{M}), \mathscr{D}\left(\pi_{\phi}\right), \lambda_{\phi}(I)\right)$ is a full standard system.

Proof. (1) It follows from Theorem 5. 1 that $\phi=\omega_{\xi_{\phi}}$ for $\xi_{\phi} \in \mathscr{P} \xi_{\xi_{0}}$ $\cap \mathscr{D}$. Since $[\mathscr{M}]_{w \sigma}^{\prime \prime}=\overline{[\mathscr{M}]^{t_{s}^{*}}}$, it follows that $\omega_{\xi_{\phi}}^{\prime \prime} \in\left(\mathscr{M}^{\prime \prime}\right)_{*}^{+}$satisfies the KMS-condition with respect to $\left\{\sigma_{t}^{\xi_{0}}\right\}$, so that by ([32] Theorem 15.4) there exists a positive self-adjoint operator $H$ affiliated with $\mathscr{M}^{\prime} \cap \mathscr{M}^{\prime \prime}$ such that

$$
\left(A \xi_{\phi} \mid \xi_{\phi}\right)=\left(A H \xi_{0} \mid H \xi_{0}\right)
$$

for all $A \in \mathscr{M}^{\prime \prime}$. We denote by $U^{\prime}$ the partial isometry on $\mathfrak{S}(\mathscr{D})$ defined by:

$$
A \xi_{\phi} \rightarrow A H \xi_{0}, A \in \mathscr{M}^{\prime \prime} .
$$

Using $[\mathscr{M}]_{w \sigma}^{\prime \prime}=\overline{[\mathscr{M}}^{t_{s}^{*}}$, we can prove $U^{\prime} \in \mathscr{M}^{\prime}$, and hence $H \xi_{0}=U^{\prime} \xi_{\phi} \in$ $\mathscr{D}$, which implies $\phi=\omega_{H \xi_{0}}$ by (5.3).

Suppose $\phi$ is faithful. Since the projection $E$ of $\mathscr{\mathcal { E }}(\mathscr{D})$ onto Ker $H$ is contained in $\mathscr{M}^{\prime} \cap \mathscr{M}^{\prime \prime}$ and $(\mathscr{M}, \mathscr{D})$ is a generalized von Neumann algebra, it follows that $E_{0} \equiv E / \mathscr{D} \in \mathscr{M}$, and hence

$$
\phi\left(E_{0}\right)=\left(E H \xi_{0} \mid H \xi_{0}\right)=0 .
$$


Since $\phi$ is faithful, we have $E_{0}=0$, and hence $H$ is nonsingular. It follows from Lemma 3.4 and Lemma 3.5 that $\phi$ is a standard positive linear functional on $\mathscr{M}$ with $\mathscr{D}_{\phi}=\mathscr{D}\left(\pi_{\phi}\right)$.

(2) We denote by $E_{H \xi_{0}}^{\prime}$ the projection of $\mathscr{S}(\mathscr{D})$ onto $\overline{\mathscr{M} H \xi_{0}}$. It

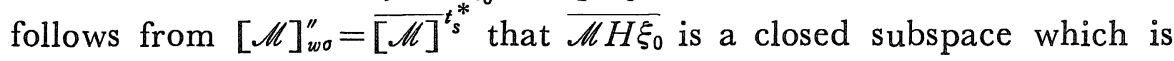
invariant for $\mathscr{M}^{\prime \prime}$, and hence $E_{H \xi_{0}}^{\prime} \in \mathscr{M}^{\prime}$. It is easily shown that the restriction $\mathscr{M} / E_{H \xi_{0}}^{\prime} \mathscr{D}$ of the $O_{p}^{*}$-algebra $\mathscr{M}$ to $E_{H \xi_{0}}^{\prime} \mathscr{D}$ is a closed $O_{P}^{*}-$ algebra such that $\left(\mathscr{M} / E_{H \xi_{0}}^{\prime} \mathscr{D}\right)^{\prime}=E_{H \xi_{0}}^{\prime} \mathscr{M}^{\prime} / E_{H \xi_{0}}^{\prime} \mathscr{S}(\mathscr{D})$ and $\left(\mathscr{M} / E_{H \xi_{0}}^{\prime} \mathscr{D}\right)^{\prime \prime}$ $=\mathscr{M}^{\prime \prime} / E_{H \xi_{0}}^{\prime} \mathfrak{S}(\mathscr{D})$. Let $H=\int_{0}^{\infty} \lambda d E(\lambda)$ be the spectral resolution of $H_{\text {。 }}$ Put

$$
K_{n}=\int_{1 / n}^{n} \frac{1}{\lambda} d E(\lambda), \quad E_{n}=\int_{1 / n}^{n} d E(\lambda)
$$

for $n \in \mathbb{N}$. Then we have $K_{n} / \mathscr{D}, E_{n} / \mathscr{D} \in \mathscr{M}$ and

$$
\begin{aligned}
\lim _{n \rightarrow \infty} E_{n} E_{H \xi_{0}}^{\prime} X \xi_{0} & =\lim _{n \rightarrow \infty} K_{n} X H \xi_{0} \\
& =E_{H \xi_{0}}^{\prime} X \xi_{0}-E(0) E_{H \xi_{0}}^{\prime} X \xi_{0} \\
& =E_{H \xi_{0}}^{\prime} X \xi_{0}, \\
\lim _{n \rightarrow \infty} Y E_{n} E_{H \xi_{0}}^{\prime} X \xi_{0} & =E_{H \xi_{0}}^{\prime} Y X \xi_{0}
\end{aligned}
$$

for each $X, Y \in \mathscr{M}$, which implies $E_{H \xi_{0}}^{\prime} X \xi_{0} \in{\overline{\mathscr{M} H \xi_{0}}}^{t} / \mathbb{M}$ for each $X \in \mathscr{M}_{\text {. }}$ On the other hand, it is easily shown that $E_{H \xi_{0}}^{\prime} \mathscr{D} \subset{\overline{E_{H \xi_{0}}^{\prime}} \mathscr{M} \xi_{0}^{t}}^{t} \mathscr{M}$. Hence, we have

$$
{\overline{E_{H \xi_{0}}^{\prime}}, \mathscr{M} \xi_{0}}^{t} \mathscr{M}={\overline{\mathscr{M} H \xi_{0}}}^{t} \mathscr{M}=E_{H \xi_{0}}^{\prime} \mathscr{D} ;
$$

that is, $H \xi_{0}$ is a strongly cyclic vector for $\left(\mathscr{M} / E_{H \xi_{0}}^{\prime} \mathscr{D}, E_{H \xi_{0}}^{\prime} \mathscr{D}\right)$. It is clear that $H \xi_{0}$ is a separating vector for $\left(\mathscr{M} / E_{H \xi_{0}}^{\prime} \mathscr{D}\right)^{\prime \prime}=\mathscr{M}^{\prime \prime} / E_{H \xi_{0}}^{\prime} \mathscr{S}_{\mathscr{E}}(\mathscr{D})$. Since $\Delta_{\xi_{0}}^{i t} \mathscr{M} H \xi_{0}=\mathscr{M} H \xi_{0}$ for all $t \in \mathbb{R}$, we have $\Delta_{\xi_{0}}^{i t} E_{H \xi_{0}}^{\prime}=E_{H \xi_{0}}^{\prime} \Delta_{\xi_{0}}^{i t}$ for all $t \in \mathbb{R}$, which implies that $\omega_{H \xi_{0}}^{\prime \prime}$ satisfies the KMS-condition with respect to a strongly continuous one-parameter group of *-automorphisms:

$$
A / E_{H \xi_{0}}^{\prime} \mathfrak{S}(\mathscr{D}) \rightarrow\left(\Delta_{\xi_{0}}^{i t} E_{H \xi_{0}}^{\prime}\right) A\left(E_{H \xi_{0}}^{\prime} \Delta_{\xi_{0}}^{-i t}\right)
$$

of the von Neumann algebra $\mathscr{M}^{\prime \prime} / E_{H \xi_{0}}^{\prime} \mathfrak{E}(\mathscr{D})$. By ([32] Theorem 13.2) we have

$$
\Delta_{H \xi_{0}}^{\prime \prime i t} A \Delta_{H \xi_{0}}^{\prime \prime} E_{H \xi_{0}}^{\prime} \xi=\left(\Delta_{\xi_{0}}^{i t} E_{H \xi_{0}}^{\prime}\right) A\left(\Delta_{\xi_{0}}^{-i t} E_{H \xi_{0}}^{\prime}\right) E_{H \xi_{0}}^{\prime} \xi
$$

for all $\xi \in \mathscr{S}_{2}(\mathscr{D})$ and $t \in \mathbb{R}$, which implies $\Delta_{H \xi_{0}}^{\prime \prime i t}=\Delta_{\xi_{0}}^{i t} E_{H \xi_{0}}^{\prime}$ for all $t \in \mathbb{R}$. Hence $H \xi_{0}$ is a modular vector for $\left(\mathscr{M} / E_{H \xi_{0}}^{\prime} \mathscr{D}, E_{H \xi_{0}}^{\prime} \mathscr{D}\right)$ with $\mathscr{D}_{H \xi_{0}}=$ 
$E_{H \xi_{0}}^{\prime} \mathscr{D}$. Since $[\mathscr{M}]_{w \sigma}^{\prime \prime}=\overline{[\mathscr{M}}^{t_{s}^{*}}$, it follows that $H \xi_{0}$ is standard, which implies by (5.4) that $\phi$ is a standard positive linear functional on $\mathscr{M}$ with $\mathscr{D}_{\phi}=\mathscr{D}\left(\pi_{\phi}\right)$.

Suppose $\phi$ is faithful. By (1), $H$ is non-singular, and so $E_{H \xi_{0}}^{\prime}=I$. By (5.4) $H \xi_{0}$ is a strongly cyclic vector for $\mathscr{M}$, and hence ( $\mathscr{M}, \mathscr{D}$, $\left.H \xi_{0}\right)$ is a full standard system, which implies that so is $\left(\pi_{\phi}(\mathscr{M})\right.$, $\left.\mathscr{D}\left(\pi_{\phi}\right), \lambda_{\phi}(I)\right)$. This completes the proof.

We can similarly prove the following result using ([32] Theorem 15.2).

Theorem 5. 6. Suppose ( $\left.\mathscr{M}, \mathscr{D}, \xi_{0}\right)$ is a standard system and $\phi \in \mathscr{M}_{*}^{+}$. Then $\phi$ is $\left\{\sigma_{t}^{\xi_{0}}\right\}$-invariant if and only if $\phi$ is represented as

$$
\phi(X)=\left(X H \xi_{0} \mid H \xi_{0}\right), X \in \mathscr{M}
$$

for some positive self-adjoint operator $H$ affliated with $\mathscr{M}^{\prime \prime \sigma^{\xi_{0}}}$ such that $\xi_{0} \in \mathscr{D}(H)$ and $H \xi_{0} \in \mathscr{D}$.

We apply Radon-Nikodym theorems obtained the above to the spatial theory for $O_{p}^{*}$-algebras. The spatial theory for $O_{p}^{*}$-algebras was investigated in $[13,16,33,35]$. In particular, it was obtained that every *-automorphism of the maximal $O_{p}^{*}$-algebra is unitarily implemented $[33,35]$ and each $*$-automorphism $\alpha$ of the $O_{p}^{*}$-algebra $\pi_{0}(\mathscr{A})$ of the Schrödinger representation $\pi_{0}$ of the canonical algebra $\mathscr{A}$ for one degree of freedom satisfying $\alpha\left(\pi_{0}(\mathscr{A})^{+}\right) \subset \pi_{0}(\mathscr{A})^{+}$is unitarily implemented [33]. In the case of von Neumann algebras $\mathscr{M}_{0}$ with a cyclic and separating vector, each $*$-automorphism of $\mathscr{M}_{0}$ is always unitarily implemented, but in [33] Takesue gave an example of the self-adjoint $O_{p}^{*}$-algebra (the polynomial algebra $\left(\mathscr{P}\left(-i \frac{d}{d t} / \mathscr{D}\right), \mathscr{D}\right)$, where $\left.\mathscr{D}=\left\{f \in C^{\infty}[0,1] ; f^{(n)}(0)=f^{(n)}(1), \quad n=0,1,2, \ldots\right\}\right)$ with a strongly cyclic and separating vector for which the above fact does not necessarily hold, and so we need consider the spatial theory for a self-adjoint $O_{p}^{*}$-algebra with a strongly cyclic and separating vector.

In a previous paper [16], we obtained the following Propositions 5. 7, 5. 8 .

Proposition 5.7. Let ( $\mathscr{M}, \mathscr{D})$ be a self-adjoint $O_{p}^{*}$-algebra, a vector $\xi_{0}$ in $\mathscr{D}$ be strongly cyclic for $\mathscr{M}$ and separating for $\mathscr{M}^{\prime \prime}$ and $\alpha$ be a 
*-automorphism of $\mathscr{M}$. Then the following statements hold.

(1) Suppose both the map $X \xi_{0} \rightarrow \alpha(X) \xi_{0}$ and $X \xi_{0} \rightarrow \alpha^{-1}(X) \xi_{0}$ are continuous. Then $\alpha$ is represented as

$$
\alpha(X)=U^{\dagger} X U, \quad X \in \mathscr{M}
$$

for some $U \in \mathscr{L}^{\dagger}(\mathscr{D})_{u} \equiv\left\{U \in \mathscr{L}^{\dagger}(\mathscr{D}) ; \bar{U}\right.$ is unitary .

(2) Suppose $\pi_{\phi_{0}+\phi_{0} \alpha}(\mathscr{M})^{\prime}$ is a von Neumann algebra, and the map $X \xi_{0} \rightarrow \alpha(X) \xi_{0}$ and $X \xi_{0} \rightarrow \alpha^{-1}(X) \xi_{0}$ are closable. Then $\alpha$ is represented as

$$
\alpha(X)=U^{\dagger} X U, \quad X \in \mathscr{M}
$$

for some $U \in \mathscr{L}^{\dagger}(\mathscr{D})_{\text {u. }}$

Throughout the rest of this section, let $(\mathscr{M}, \mathscr{D})$ be a selfadjoint $O_{p}^{*}$-algebra such that $\left[\mathscr{M}^{t_{s}^{*}}=[\mathscr{M}]_{w o}^{\prime \prime}\right.$, a vector $\xi_{0}$ in $\mathscr{D}$ be strongly cyclic for $\mathscr{M}$ and separating for $\mathscr{M}^{\prime \prime}$ and $\alpha$ be a $*$-automorphism of M.

Proposition 5. 8. Suppose $\phi_{0} \circ \alpha$ and $\phi_{0} \circ \alpha^{-1}$ in $\mathscr{M}_{*}^{+}$; in particular, $\alpha$ and $\alpha^{-1}$ are continuous relative to the $\sigma$-weak topology for $\mathscr{M}$. Then $\alpha$ is represented as

$$
\alpha(X)=U^{\dagger} X U, \quad X \in \mathscr{M}
$$

for some $U \in \mathscr{L}^{\dagger}(\mathscr{D})_{u}$.

We here weaken the condition of the continuity of $\alpha$ and $\alpha^{-1}$ in Proposition 5.8.

Theorem 5.9. Suppose $\alpha$ is continuous relative to the $\sigma$-strong topology for $\mathscr{M}$. Then $\alpha$ is represented as

$$
\alpha(X)=U^{\dagger} X U, \quad X \in \mathscr{M}
$$

for some $U \in \mathscr{L}^{\dagger}(\mathscr{D})_{i} \equiv\left\{U \in \mathscr{L}^{\dagger}(\mathscr{D}) ; U^{*} \bar{U}=I\right\}$. Further, suppose $\alpha^{-1}$ is closable relative to the $\sigma$-strong* topology for $\mathscr{M}$. Then $\alpha$ is represented as

$$
\alpha(X)=U^{\dagger} X U, \quad X \in \mathscr{M}
$$

for some $U \in \mathscr{L}^{\dagger}(\mathscr{D})_{u}$.

Proof. Since $\alpha$ is continuous relative to the $\sigma$-strong topology for 
$\mathscr{M}$, it follows that

$$
\left\|\alpha(X) \xi_{0}\right\| \leqq\left\|[X]\left\{\xi_{n}\right\}\right\|, \quad X \in \mathscr{M}
$$

for some $\left\{\xi_{n}\right\} \in \mathscr{D}^{\infty}(\mathscr{M})$. In similar to the proof of ([16] Lemma 5.2), we can prove that

$$
\left(\alpha(X) \xi_{0} \mid \xi_{0}\right)=\left(X \zeta_{0} \mid \zeta_{0}\right), \quad X \in \mathscr{M}
$$

for some $\zeta_{0} \in \mathscr{D}$. By Theorem 5.1 there exists a vector $\eta_{0}$ in $\mathscr{P} \xi_{0} \cap \mathscr{D}$ such that

$$
\left(\alpha(X) \xi_{0} \mid \xi_{0}\right)=\left(X \eta_{0} \mid \eta_{0}\right)
$$

for all $X \in \mathscr{M}$. Put

$$
U_{0} \alpha(X) \xi_{0}=X \eta_{0}, \quad X \in \mathscr{M} .
$$

Then, by (5.5) the closure $\overline{U_{0}}$ of $U_{0}$ is an isometry on $\mathscr{S}(\mathscr{D})$. We now put

$$
U=\overline{U_{0}} / \mathscr{D} .
$$

Then it is easily shown that $U \in \mathscr{L}^{\dagger}(\mathscr{D})_{i}, \bar{U} U^{*} \in \mathscr{M}^{\prime}$ and $\alpha(X)=U^{\dagger} X U$ for all $X \in \mathscr{M}$.

Suppose $\alpha^{-1}$ is closable relative to the $\sigma$-strong* topology. Then we show $U \in \mathscr{L}^{\dagger}(\mathscr{D})_{u}$. Suppose $A \eta_{0}=0, A \in \mathscr{M}^{\prime \prime}$. Since $\left.\left[\mathscr{M}^{\prime \prime}\right] \subset \overline{[\mathscr{M}}\right]^{t_{s}^{*}}$, there exists a net $\left\{X_{\lambda}\right\}$ in $\mathscr{M}$ such that

$$
\lim _{\lambda}\left[X_{\lambda}\right]\left\{\xi_{n}\right\}=[A]\left\{\xi_{n}\right\}, \lim _{\lambda}\left[X_{\lambda}^{\dagger}\right]\left\{\xi_{n}\right\}=\left[A^{*}\right]\left\{\xi_{n}\right\}
$$

for each $\left\{\xi_{n}\right\} \in \mathscr{D}^{\infty}(\mathscr{M})$. Since $\alpha$ is continuous relative to the $\sigma$-strong topology for $\mathscr{M}$, there exist elements $\alpha^{\prime \prime}(A)$ and $\alpha^{\prime \prime}\left(A^{*}\right)$ of $\mathscr{L}(\mathscr{D}$, $\mathscr{E}(\mathscr{D})$ ) such that

$$
\begin{aligned}
& \lim _{\lambda}\left[\alpha\left(X_{\lambda}\right)\right]\left\{\xi_{n}\right\}=\left[\alpha^{\prime \prime}(A)\right]\left\{\xi_{n}\right\}, \\
& \lim _{\lambda}\left[\alpha\left(X_{\lambda}^{\dagger}\right)\right]\left\{\xi_{n}\right\}=\left[\alpha^{\prime \prime}\left(A^{*}\right)\right]\left\{\xi_{n}\right\}
\end{aligned}
$$

for each $\left\{\xi_{n}\right\} \in \mathscr{D}^{\infty}(\mathscr{M})$. Then we have

$$
\begin{aligned}
\left(\left[\alpha^{\prime \prime}(A)\right]\left\{\xi_{n}\right\} \mid\left\{\eta_{n}\right\}\right) & =\lim _{\lambda}\left(\left[\alpha\left(X_{\lambda}\right)\right]\left\{\xi_{n}\right\} \mid\left\{\eta_{n}\right\}\right) \\
& =\lim _{\lambda}\left(\left\{\xi_{n}\right\} \mid\left[\alpha\left(X_{\lambda}^{\dagger}\right)\right]\left\{\eta_{n}\right\}\right) \\
& =\left(\left\{\xi_{n}\right\} \mid\left[\alpha^{\prime \prime}\left(A^{*}\right)\right]\left\{\eta_{n}\right\}\right)
\end{aligned}
$$

for each $\left\{\xi_{n}\right\},\left\{\eta_{n}\right\} \in \mathscr{D}^{\infty}(\mathscr{M})$. Hence, we have $\alpha^{\prime \prime}(A) \in \mathscr{C}^{\dagger}(\mathscr{D}, \mathfrak{S}(\mathscr{D}))$ and $\alpha^{\prime \prime}(A)^{\dagger}=\alpha^{\prime \prime}\left(A^{*}\right)$. By (5.5), (5.6) and (5.7) we have 


$$
\begin{aligned}
\left\|\alpha^{\prime \prime}(A) \xi_{0}\right\| & =\lim _{\lambda}\left\|\alpha\left(X_{\lambda}\right) \xi_{0}\right\| \\
& =\lim _{\lambda}\left\|X_{\lambda} \eta_{0}\right\| \\
& =\left\|A \eta_{0}\right\|=0,
\end{aligned}
$$

and hence $\alpha^{\prime \prime}(A) \xi_{0}=0$, and further we have

$$
\begin{aligned}
\left(\alpha^{\prime \prime}\left(A^{*}\right) \xi_{0} \mid C \xi_{0}\right) & =\lim _{\lambda}\left(\alpha\left(X_{\lambda}^{\uparrow}\right) \xi_{0} \mid C \xi_{0}\right) \\
& =\lim _{\lambda}\left(C^{*} \xi_{0} \mid \alpha\left(X_{\lambda}\right) \xi_{0}\right) \\
& =\left(C^{*} \xi_{0} \mid \alpha^{\prime \prime}(A) \xi_{0}\right) \\
& =0
\end{aligned}
$$

for each $C \in \mathscr{M}^{\prime}$, and hence $\alpha^{\prime \prime}\left(A^{*}\right) \xi_{0}=0$, which implies

$$
\left(\alpha^{\prime \prime}(A) \xi \mid C \xi_{0}\right)=\left(C^{*} \xi_{0} \mid \alpha^{\prime \prime}\left(A^{*}\right) \xi_{0}\right)=0
$$

for each $\xi \in \mathscr{D}$ and $C \in \mathscr{M}^{\prime}$. Hence, $\alpha^{\prime \prime}(A)=0$. By (5.6) and (5.7), a net $\left\{\alpha\left(X_{\lambda}\right)\right\}$ in $\mathscr{M}$ converges to 0 and $\left\{\alpha^{-1}\left(\alpha\left(X_{\lambda}\right)\right)\right\}$ is a Cauchy net in $\mathscr{M}$ relative to the $\sigma$-strong* topology for $\mathscr{M}$. Since $\alpha^{-1}$ is closable relative to the $\sigma$-strong* topology for $\mathscr{M}$, it follows that $\lim _{\lambda} X_{\lambda}=0$, and hence $A=0$. Hence, $\eta_{0}$ is a separating vector for $\mathscr{M}^{\prime \prime}$. It follows from $\eta_{0} \in \mathscr{P}_{\xi_{0}}$ that $\eta_{0}$ is a cyclic vector for $\mathscr{M}^{\prime \prime}$, which implies that $\bar{U}$ is a unitary operator on $\mathscr{S}(\mathscr{D})$. This completes the proof.

Theorem 5. 10. Suppose $\phi_{0} \circ \alpha \in \mathscr{M}_{*}^{+}$and the map $X \xi_{0} \rightarrow \alpha^{-1}(X) \xi_{0}$ is closable. Then $\alpha$ is represented as

$$
\alpha(X)=U^{\dagger} X U, \quad X \in \mathscr{M}
$$

for some $U \in \mathscr{L}^{\uparrow}(\mathscr{D})_{u}$.

Proof. By Theorem 5.1 there exists an element $\eta_{0}$ of $\mathscr{P} \xi_{0} \cap \mathscr{D}$ such that

$$
\left(\alpha(X) \xi_{0} \mid \xi_{0}\right)=\left(X \eta_{0} \mid \eta_{0}\right)
$$

for all $X \in \mathscr{M}$. Suppose $A \eta_{0}=0, A \in \mathscr{M}^{\prime \prime}$. Since $\left[\mathscr{M}^{\prime \prime}\right] \subset \overline{\left[\mathscr{M}^{t_{s}^{*}}\right.}$ and (5.8), there exists a net $\left\{X_{\lambda}\right\}$ in $\mathscr{M}$ such that

$$
\lim _{\lambda} \alpha\left(X_{\lambda}\right) \xi_{0}=0 \text { and } \lim _{\lambda} \alpha^{-1}\left(\alpha\left(X_{\lambda}\right)\right) \xi_{0}=A \xi_{0}
$$

Since $X \xi_{0} \rightarrow \alpha^{-1}(X) \xi_{0}$ is closable, we have $A \xi_{0}=0$, and hence $A=0$. Hence, $\eta_{0}$ is a separating vector for $\mathscr{M}^{\prime \prime}$. It follows from $\eta_{0} \in \mathscr{P}_{\xi_{0}}$ that $\eta_{0}$ is a cyclic vector for $\mathscr{M}^{\prime \prime}$, which implies by (5.8) that $\alpha$ is represented as 


$$
\alpha(X)=U^{\dagger} X U, \quad X \in \mathscr{M}
$$

for some $U \in \mathscr{L}^{\dagger}(\mathscr{D})_{u}$.

\section{§6. Examples}

In this section we investigate the absolute continuity and singularity of positive linear functionals on the $O_{p}^{*}$-algebra generated by the differential operator, the $O_{p}^{*}$-algebra defined by the Schrödinger representation and the maximal $O_{p}^{*}$-algebra $\mathscr{L}^{\dagger}(\mathscr{S}(\mathbb{R}))$ on the Schwartz space $\mathscr{S}(\mathbf{R})$.

Example 6.1. Put

$$
\begin{aligned}
& \mathscr{D}=\left\{\xi \in C^{\infty}[0,1] ; \xi^{(n)}(0)=\xi^{(n)}(1), n=0,1,2, \ldots\right\}, \\
& X_{0}=-i \frac{d}{d t} \mid \mathscr{D} \\
& \left.\xi_{0}(t)=\left[\exp \left\{-\exp \left(-\frac{d^{2}}{d t^{2}}\right)\right\}\right] 5-4 \cos 2 \pi t\right)^{-1}, \quad t \in[0,1] .
\end{aligned}
$$

Then the polynomial algebra $\mathscr{P}\left(X_{0}\right)$ generated by $X_{0}$ is a self-adjoint $O_{p}^{*}$-algebra on $\mathscr{D}$ and a vector $\xi_{0}$ in $\mathscr{D}$ is strongly cyclic for $\mathscr{P}\left(X_{0}\right)$ and separating for $\mathscr{P}\left(X_{0}\right)^{\prime \prime}$. We consider positive linear functionals on $\mathscr{P}\left(X_{0}\right)$ defined by

$$
\phi_{a}^{b}\left(p\left(X_{0}\right)\right)=\left(p\left(a X_{0}+b\right) \xi_{0} \mid \xi_{0}\right), \quad a \neq 0, b \in \mathbb{R} .
$$

Then the following statements hold.

(1) $\phi_{n}^{2 \pi m}(n \neq 0, m \in \mathbb{Z})$ are strongly $\omega_{\xi_{0}}$-absolutely continuous.

In fact, by ([33] Example) $\phi_{n}^{2 \pi m}$ is represented as

$$
\phi_{n}^{2 \pi m}\left(p\left(X_{0}\right)\right)=\left(p\left(X_{0}\right) U \xi_{0} \mid U \xi_{0}\right)
$$

for some $U \in \mathscr{L}^{\dagger}(\mathscr{D})_{i} \equiv\left\{U \in \mathscr{L}^{\dagger}(\mathscr{D}) ; U^{*} \bar{U}=I\right\}$. We put

$$
\left(\phi_{n}^{2 \pi m}\right)^{\prime \prime}(A)=\left(A U \xi_{0} \mid U \xi_{0}\right), \quad A \in \mathscr{P}\left(X_{0}\right)^{\prime \prime} .
$$

Since $\mathscr{P}\left(X_{0}\right)^{\prime \prime}$ is a commutative von Neumann algebra ([15] Theorem 2.1 and [25] Theorem 7.1) and $\xi_{0}$ is a cyclic vector for $\mathscr{P}\left(X_{0}\right)^{\prime \prime}$, it follows that $\mathscr{P}\left(X_{0}\right)^{\prime \prime}$ is finite, so that by ([19] Corollary 2.3) $\left(\phi_{n}^{2 \pi m}\right)^{\prime \prime}$ is strongly $\omega_{\xi_{0}}-$ absolutely continuous. Hence we have

$$
\left(A U \xi_{0} \mid U \xi_{0}\right)=\left(\phi_{n}^{2 \pi m}\right)^{\prime \prime}(A)=\left(A H^{\prime} \xi_{0} \mid H^{\prime} \xi_{0}\right), \quad A \in \mathscr{P}\left(X_{0}\right)^{\prime \prime}
$$

for some positive self-adjoint operator $H^{\prime}$ in $L^{2}[0,1]$ affiliated with $\mathscr{P}\left(X_{0}\right)^{\prime}$, which implies 


$$
H^{\prime} \xi_{0} \in \mathscr{D} \text { and }\left(\phi_{n}^{2 \pi m}\right)\left(p\left(X_{0}\right)\right)=\left(p\left(X_{0}\right) H^{\prime} \xi_{0} \mid H^{\prime} \xi_{0}\right) 。
$$

Hence, $\phi_{n}^{2 \pi m}$ is strongly $\omega_{\xi_{0}}-$ absolutely continuous.

(2) For each bounded subset $B$ of $\mathbb{R}$ we define positive linear functionals on $\mathscr{P}\left(X_{0}\right)$ by

$$
\begin{aligned}
& \left(\omega_{\xi_{0}} \circ \chi_{B}\right)\left(p\left(X_{0}\right)\right)=\left(\chi_{B}\left(X_{0}\right) p\left(X_{0}\right) \xi_{0} \mid \xi_{0}\right), \\
& \left(\phi_{a}^{b} \circ \chi_{B}\right)\left(p\left(X_{0}\right)\right)=\left(\chi_{B}\left(X_{0}\right) p\left(a X_{0}+b\right) \xi_{0} \mid \xi_{0}\right) .
\end{aligned}
$$

Then $\phi_{a}^{b} \circ \chi_{B}(a \in \mathbb{Z}$ or $b \notin 2 \pi \mathbb{Z})$ are $\left(\omega_{\xi_{0}} \circ \chi_{B}\right)$-singular.

In fact, for each polynomial $p$ and $n \in \mathbb{N}$ we define a polynomial $p_{n}$ by

$$
\begin{gathered}
p_{n}(t)=\sum_{k=1}^{2 n+1} \alpha_{k}\{(t+2 n \pi)(t+2(n-1) \pi) \ldots(t+2 \pi) t(t-2 \pi) \\
\ldots(t-2 n \pi)\}^{k}
\end{gathered}
$$

where $\left\{\alpha_{1}, \alpha_{2}, \ldots, \alpha_{2 n+1}\right\}$ is a unique solution of the equation:

$$
p_{n}(2 m \pi a+b)=p(2 m \pi a+b), m=-n, \ldots,-1,0,1, \ldots, n
$$

(the existence of the unique solution dues to $a \neq 0$ ). Since $B$ is a bounded subset of $\mathbb{R}$, it follows that

$$
\begin{aligned}
& \left(\omega_{\xi_{0}} \circ \chi_{B}\right)\left(p_{n}\left(X_{0}\right)^{\dagger} p_{n}\left(X_{0}\right)\right)=0, \\
& \left(\phi_{a}^{b} \circ \chi_{B}\right)\left(\left(p_{n}\left(X_{0}\right)-p\left(X_{0}\right)\right)^{\dagger}\left(p_{n}\left(X_{0}\right)-p\left(X_{0}\right)\right)=0\right.
\end{aligned}
$$

for sufficient large all $n \in \mathbb{N}$. Hence, $\phi_{a}^{b} \circ \chi_{B}$ is $\omega_{\xi_{0}} \circ \chi_{B}-$ singular.

Let $\mathscr{S}=\mathscr{S}(\mathbb{R})$ be the Schwartz space of infinitely differentiable rapidly decreasing functions and $\left\{f_{n}\right\}_{n=0,1,2, \ldots}$ be an orthonormal basis in the Hilbert space $L^{2}=L^{2}(\mathbb{R})$ contained in $\mathscr{S}$ consisting of the normalized Hermite functions. We denote by $L^{2} \otimes \overline{L^{2}}$ the Hilbert space with inner product $\langle\mid\rangle$ of Hilbert-Schmidt operators on $L^{2}$, by $\mathscr{S} \otimes \overline{L^{2}}$ the subspace $\left\{T \in L^{2} \otimes \overline{L^{2}} ; T L^{2} \subset \mathscr{S}\right\}$ of $L^{2} \otimes \overline{L^{2}}$ and by $\left(\mathscr{S} \otimes L_{2}\right)+$ the set of all positive operators of $\mathscr{S} \otimes \overline{L^{2}}$. Let $K$ be a densely defined closed operator in $L^{2}$. We define densely defined closed operators $\pi^{\prime \prime}(K)$ and $\pi^{\prime}(K)$ as follows:

$$
\begin{aligned}
& \left\{\begin{array}{l}
\mathscr{D}\left(\pi^{\prime \prime}(K)\right)=\left\{T \in L^{2} \otimes \overline{L^{2}} ; \quad K T \in L^{2} \otimes \overline{L^{2}}\right\}, \\
\pi^{\prime \prime}(K) T=K T, \quad T \in \mathscr{D}\left(\pi^{\prime \prime}(K)\right) ;
\end{array}\right. \\
& \left\{\begin{array}{l}
\mathscr{D}\left(\pi^{\prime}(K)\right)=\left\{T \in L^{2} \otimes \overline{L^{2}} ; \overline{T K} \in L^{2} \otimes \overline{L^{2}}\right\}, \\
\pi^{\prime}(K)=\overline{T K}, \quad T \in \mathscr{D}\left(\pi^{\prime}(K)\right) .
\end{array}\right.
\end{aligned}
$$


Then $\pi^{\prime \prime}(K)$ (resp. $\pi^{\prime}(K)$ ) is a densely defined closed operator in $L^{2} \otimes \overline{L^{2}}$ affiliated with the von Neumann algebra $\pi^{\prime \prime}\left(\mathscr{B}\left(L^{2}\right)\right.$ ) (resp. $\left.\pi^{\prime \prime}\left(\mathscr{B}\left(L^{2}\right)\right)^{\prime}=\pi^{\prime}\left(\mathscr{B}\left(L^{2}\right)\right)\right)$. In particular, if $K$ is a positive selfadjoint operator in $L^{2}$, then $\pi^{\prime \prime}(K)$ and $\pi^{\prime}(K)$ are positive self-adjoint operators in $L^{2} \otimes \overline{L^{2}}$ ([14] Lemma 5.1).

As stated in Section 3, a self-adjoint representation $\pi$ of $\mathscr{L}^{\dagger}(\mathscr{S})$ in $L^{2} \otimes \bar{L}^{2}$ is defined by

$$
\pi(X) T=X T, \quad X \in \mathscr{L}^{\dagger}(\mathscr{S}), \quad T \in \mathscr{S} \otimes \overline{L^{2}},
$$

which satisfies

$$
\pi\left(\mathscr{L}^{\dagger}(\mathscr{S})\right)^{\prime}=\pi^{\prime}\left(\mathscr{B}\left(L^{2}\right)\right) \text { and } \pi\left(\mathscr{L}^{\dagger}(\mathscr{S})\right)^{\prime \prime}=\pi^{\prime \prime}\left(\mathscr{B}\left(L^{2}\right)\right) .
$$

We put

$$
\begin{aligned}
& \boldsymbol{s}_{+}=\left\{\left\{\alpha_{n}\right\} ; \alpha_{n}>0 \text { for } n=0,1,2, \ldots\right. \text { and } \\
&\left.\sup ^{n} n^{k} \alpha_{n}<\infty \text { for each } k \in \mathbf{N}\right\}, \\
& \Omega_{\left\{\alpha_{n}\right\}}= \sum_{n=0}^{\infty} \alpha_{n} f_{n} \otimes \overline{f_{n}}, \quad\left\{\alpha_{n}\right\} \in \boldsymbol{s}_{+} .
\end{aligned}
$$

Then, for each $\left\{\alpha_{n}\right\} \in \boldsymbol{s}_{+}\left(\pi\left(\mathscr{L}^{\dagger}(\mathscr{S})\right), \mathscr{S} \otimes \overline{L^{2}}, \Omega_{\left\{\alpha_{n}\right]}\right)$ is a full standard system such that $J_{\Omega_{\left\{\alpha_{n}\right\}}} T=T^{*}$ for $T \in L^{2} \otimes L^{2}, \Delta_{\Omega_{\left\{\alpha_{n}\right\}}}=\pi^{\prime}\left(\Omega_{\left\{\alpha_{n}\right\}}^{-2}\right) \pi^{\prime \prime}\left(\Omega_{\left\{\alpha_{n}\right\}}^{2}\right)$ and $\left\{\sigma_{t}^{\Omega_{\left\{\left(\alpha_{n}\right\}\right.}}(\cdot) \equiv \Omega_{\left\{\alpha_{n}\right\}}^{2 i t} \circ \Omega_{\left\{\alpha_{n}\right\}}^{-2 i t}\right\}_{t \in \mathbf{R}}$ is a one-parameter group of $*$-automorphisms of $\mathscr{L}^{\dagger}(\mathscr{S})$ satisfying $\Delta_{Q_{\left\{\alpha_{n}\right\}}}^{i t} \pi(X) \Delta_{\Omega_{\left\{\alpha_{n}\right\}}^{-i t}}^{-i}=\pi\left(\sigma_{t}^{\Omega_{\left\{\alpha n_{n}\right\}}}(X)\right)$ for each $X \in \mathscr{L}^{\dagger}(\mathscr{S})$ and $t \in \mathbb{R}$ ([14] Theorem 5.4, Corollary 5.5). We define strongly positive linear functionals $\phi_{\rho}\left(\rho \in \mathscr{S} \otimes \overline{L^{2}}\right)$ on $\mathscr{L}^{\dagger}(\mathscr{L})$ by

$$
\phi_{\rho}(X)=\operatorname{Tr} \rho \rho^{*} X=\langle\pi(X) \rho \mid \rho\rangle, \quad X \in \mathscr{L}^{\dagger}(\mathscr{S})
$$

and in particular, when $\rho=\Omega_{\left\{\alpha_{n}\right\}}\left(\left\{\alpha_{n}\right\} \in \boldsymbol{s}_{+}\right)$we simply write $\phi_{\Omega_{\left\{\alpha_{n}\right\}}}$ by $\phi_{\left\{\alpha_{n}\right\}}$.

Let $\pi_{1}$ be a self-adjoint representation of the canonical algebra $\mathscr{A}$ for one degree of freedom defined by

$$
\pi_{1}(x)=\pi\left(\pi_{0}(x)\right), \quad x \in \mathscr{A},
$$

where $\pi_{0}$ denotes the Schrödinger representation of $\mathscr{A}$. Then $\Omega_{{ }^{\left.-e^{-n \beta}\right\}}}$ is a standard vector for $\left(\pi_{1}(\mathscr{A}), \mathscr{S} \otimes \overline{L^{2}}\right)$ with $\left(\mathscr{S} \otimes \overline{L^{2}}\right)_{\Omega_{\left(e^{-n \beta}\right)}}=\mathscr{S} \otimes \overline{L^{2}}$ and there exists a one-parameter group $\left\{\Delta_{l e}^{i t}-n \beta_{1}\right\}_{t \in \mathbf{R}}$ of $*$-automorphisms of $\mathscr{A}$ such that

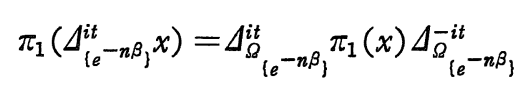


for each $x \in \mathscr{A}$ and $t \in \mathbb{R}$ ([10] Theorem 20 and [14] Corollary 5.6). For each $\rho \in\left(\mathscr{S} \otimes \overline{L^{2}}\right)$ + we simply denote by $\phi_{\rho}$ a positive linear functional $\phi_{\rho} \circ \pi_{0}$ on $\mathscr{A}$ and in particular, denote by $\phi_{\left\{\alpha_{n}\right\}}$ a positive linear functional $\phi_{\left\{\alpha_{n}\right)^{\circ}} \pi_{0}$ on $\mathscr{A}$.

In next Example 6.2 we consider the strongly $\phi_{\left\{e^{-n \beta_{\}}}\right.}$-absolute continuity, $\phi_{e^{-n \beta_{\}}}}$-singularity and $\left\{\Delta_{\left\{e^{-n \beta_{\}}}\right.}^{i t}\right\}_{t \in \mathbf{R}}$-invariance of positive linear functionals on $\mathscr{A}$, and in Example 6.3 we give concrete examples of $\phi_{\left\{e^{-n \beta_{\}}}\right.}$-singular positive linear functionals on $\mathscr{L}^{\dagger}(\mathscr{S})$ and strongly $\phi_{t_{e}^{-n \beta_{3}}}$-absolutely continuous positive linear functionals on $\mathscr{L}^{\dagger}(\mathscr{S})$, and characterize $\left\{\Delta_{\left\{e^{i t}\right\}}^{i \beta_{\}}}\right\}_{t \in \mathbf{R}}$-invariant positive linear functionals on $\mathscr{L}^{\uparrow}(\mathscr{S})$.

Example 6.2. Let $\phi$ be a positive linear functional on $\mathscr{A}$. It is well-known that $\phi \circ \pi_{0}^{-1}$ is strongly positive if and only if $\phi=\phi_{\rho}$ for some $\rho \in\left(\mathscr{S} \otimes \overline{L^{2}}\right)+[29]$. Consider positive linear functionals $\phi_{\rho}$.

(1) Suppose $\Omega_{\left\{e^{-n \beta_{3}}\right.}^{-1} \rho$ is densely defined. Then $\phi_{\rho}$ is strongly $\phi_{t^{-n \beta^{-}}}-$ absolutely continuous.

In fact, $\phi_{\rho}$ is represented as

$$
\phi_{\rho}(x)=\left\langle\pi_{1}(x)\left|\pi^{\prime}\left(\Omega_{l e^{-n \beta_{i}}}^{-1} \rho\right)\right| \Omega_{\left(e^{-n \beta_{\}}} \mid\right.}\left|\pi^{\prime}\left(\Omega_{l e^{-n \beta_{j}}}^{-1} \rho\right)\right| \Omega_{\left(e^{-n \beta_{\}}}\right.}\right\rangle, \quad x \in \mathscr{A}
$$

for a positive self-adjoint operator $\left|\pi^{\prime}\left(\Omega_{l e^{-n \beta_{\gamma}}}^{-1} \rho\right)\right|$ affliated with $\pi^{\prime}\left(\mathscr{B}\left(L^{2}\right)\right)$ such that $\left|\pi^{\prime}\left(\Omega_{\left\{e^{-n \beta}\right.}^{-1} \rho\right)\right| \Omega_{\left\{e^{-n \beta_{3}}\right.} \in \mathscr{S} \otimes \overline{L^{2}}$. Hence, $\phi_{\rho}$ is strongly $\phi_{\left\{e^{-n \beta_{\}}}\right.}$ absolutely continuous.

We next consider when $\phi_{\rho}$ is $\left\{\Delta_{\left\{e^{-n \beta_{\}}}\right.}^{i t}\right\}_{t \in \mathbb{R}}$-invariant. It is clear that $\phi_{\left\{\alpha_{n}\right\}}\left(\left\{\alpha_{n}\right\} \in s_{+}\right)$are $\left\{\Delta_{\left\{e^{-n \beta}\right\}}^{i t}\right\}$-invariant. Hence, the following question arises: If $\phi_{\rho}$ is $\left\{\Delta_{\left\{e^{-n \beta}\right\}}^{i t}\right\}$-invariant, then $\phi_{\rho}=\phi_{\left\{\alpha_{n}\right\}}$ for $\left\{\alpha_{n}\right\} \in \mathfrak{s}_{+}$? For this problem the following fact holds.

(2) If $\phi_{\rho}$ is $\phi_{\left\{e^{-n \beta_{\}}}\right.}$-dominated, then $\phi_{\rho}=\phi_{\left\{\alpha_{n}\right\}}$ for some $\left\{\alpha_{n}\right\} \in s_{+}$.

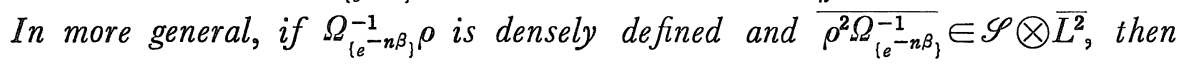
$\phi_{\rho}=\phi_{\left\{\alpha_{n}\right\}}$ for some $\left\{\alpha_{n}\right\} \in s_{+}$.

In fact, we now suppose $\Omega_{\left\{e^{-1}-n\right\}}^{-\rho} \rho$ is densely defined and $\overline{\rho^{2} \Omega_{\left\{e^{-n}\right\}}^{-1}}$ $\in \mathscr{S} \otimes \overline{L^{2}}$ and put

$$
H_{0}=\left(\Omega_{\left(e^{-n \beta}\right\}}^{-1} \rho\right)\left(\Omega_{\left(e^{-n \beta}\right\}}^{-1} \rho\right) *
$$

Then $\pi^{\prime}\left(H_{0}\right)$ is a positive self-adjoint operator in $L^{2}$ affiliated with $\pi^{\prime}\left(\mathscr{B}\left(L^{2}\right)\right)$. Since $\overline{\rho^{2} \Omega_{\left(e^{-n \beta_{\}}}\right.}^{-1}} \in \mathscr{S} \otimes \overline{L^{2}}$, it follows that 


$$
\begin{aligned}
& \Omega_{{ }^{-n \beta_{3}} \in} \in \mathscr{D}\left(\pi^{\prime}\left(H_{0}\right)\right) \text { and } \\
& \pi^{\prime}\left(H_{0}\right) \Omega_{\left[e^{-n \beta}\right\}}=\overline{\rho^{2} \Omega_{\left(e^{-n \beta_{1}}\right.}} \in \mathscr{S} \otimes \overline{L^{2}},
\end{aligned}
$$

and hence

$$
\begin{aligned}
& \pi_{1}(\mathscr{A}) \Omega_{\left\{e^{-n \beta_{\}}}\right.} \subset \mathscr{D}\left(\pi^{\prime}\left(H_{0}\right)\right), \\
& \pi^{\prime}\left(H_{0}\right) \pi_{1}(x) \Omega_{\left\{e^{-n \beta_{\}}}\right.}=\pi_{1}(x) \pi^{\prime}\left(H_{0}\right) \Omega_{\left\{e^{-n \beta_{3}}\right.}
\end{aligned}
$$

and

$$
\phi(x)=\left\langle\pi_{1}(x) \pi^{\prime}\left(H_{0}\right) \Omega_{\left\{e^{-n \beta_{\}}}\right.} \mid \Omega_{\left\{e^{-n \beta_{\}}}\right.}\right\rangle
$$

for all $x \in \mathscr{A}$. Since $\phi$ is $\left\{\Delta_{\left\{e^{-n \beta}\right\}}^{i t}\right\}$-invariant, it follows that

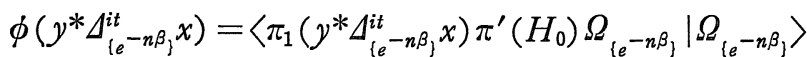

$$
\begin{aligned}
& =\left\langle\pi^{\prime}\left(H_{0}\right) \pi_{1}\left(\Delta_{\left\{e^{-n \beta_{1}}\right.}^{i t} x\right) \Omega_{\left\{e^{-n \beta_{\}}}\right.} \mid \pi_{1}(y) \Omega_{\left\{e^{-n \beta_{1}}\right.}\right\rangle \\
& =\left\langle\pi^{\prime}\left(H_{0}\right) \Delta_{\Omega_{\left\{e^{-n \beta}\right.}}^{i t} \pi_{1}(x) \Omega_{\left[e^{-n \beta_{\}}}\right.} \mid \pi_{1}(y) \Omega_{\left(e^{-n \beta_{\}}}\right.}\right\rangle \text {, } \\
& \phi\left(y^{*} \Delta_{\left[e^{-n \beta_{3}}\right.}^{i t} x\right)=\phi\left(\Delta_{\left[e^{-n \beta_{\}}}\right.}^{-i t}\left(y^{*} \Delta_{\left\{e^{-n \beta_{3}}\right.}^{i t} x\right)\right) \\
& =\phi\left(\left(\Delta_{\left\{e^{-n \beta_{1}}\right.}^{-i t} y\right) * x\right) \\
& =\left\langle\Delta_{\Omega_{\left\{e^{-n \beta}\right\}}^{i t}} \pi_{1}(x) \pi^{\prime}\left(H_{0}\right) \Omega_{\left\{e^{-n \beta_{\}}}\right.} \mid \pi_{1}(y) \Omega_{\left\{e^{-n \beta_{\}}}\right.}\right\rangle \\
& =\left\langle\Delta_{\Omega_{\{e} i \beta_{\}}}^{i t} \pi^{\prime}\left(H_{0}\right) \pi_{1}(x) \Omega_{\left\{e^{-n \beta_{\}}}\right.} \mid \pi_{1}(y) \Omega_{\left\{e^{-n \beta_{\}}}\right.}\right\rangle
\end{aligned}
$$

for all $x, y \in \mathscr{A}$, which implies since $\pi^{\prime \prime}\left(\mathscr{B}\left(L^{2}\right)\right) \Omega_{{ }_{i e}-n \beta_{\}}} \subset \mathscr{D}\left(\pi^{\prime}\left(H_{0}\right)\right)$ that

$$
\begin{aligned}
& \left\langle\pi_{1}(x) \Omega_{\left\{e^{-n \beta_{\}}}\right.} \mid \Delta_{\Omega_{\left\{e^{-n \beta_{\}}}\right.}^{i t}} \pi^{\prime}\left(H_{0}\right) \pi^{\prime \prime}(A) \Omega_{\left\{e^{-n \beta_{\}}}\right.}\right\rangle \\
& =\left\langle\pi^{\prime}\left(H_{0}\right) \Delta_{\Omega_{\left\{e^{-n \beta_{1}}\right.}^{-i t}} \pi_{1}(x) \Omega_{\left\{e^{-n \beta_{\}}}\right.} \mid \pi^{\prime \prime}(A) \Omega_{\left\{e^{-n \beta_{\}}}\right.}\right\rangle
\end{aligned}
$$

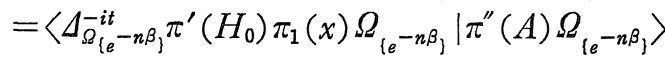

$$
\begin{aligned}
& =\left\langle\pi_{1}(x) \Omega_{i^{-n \beta_{1}}} \mid \pi^{\prime}\left(H_{0}\right) \Delta_{\Omega_{\left(e^{-n \beta}\right.}} \pi^{\prime \prime}(A) \Omega_{{ }^{-n \beta^{-n}}}\right\rangle
\end{aligned}
$$

for all $A \in \mathscr{B}\left(L^{2}\right), x \in \mathscr{A}$ and $t \in \mathbb{R}$. Hence we have

$$
\begin{aligned}
\pi^{\prime \prime}\left(\Omega_{l e^{-n \beta_{\}}}}^{2 i t}\right) \pi^{\prime}\left(\Omega_{\left(e^{-n \beta_{\}}}\right)}^{-2 i t}\right) \pi^{\prime}\left(H_{0}\right) \pi^{\prime \prime}(A) \Omega_{i e^{-n \beta_{\}}}} \\
=\pi^{\prime}\left(H_{0}\right) \pi^{\prime \prime}\left(\Omega_{\left\{e^{-n \beta_{\}}}\right.}^{2 i t}\right) \pi^{\prime}\left(\Omega_{l e^{-n \beta_{\}}}}^{-2 i t}\right) \pi^{\prime \prime}(A) \Omega_{\left\{e^{-n \beta_{\}}}\right.}
\end{aligned}
$$

for all $A \in \mathscr{B}\left(L^{2}\right)$ and $t \in \mathbb{R}$. Since $f_{k} \in \mathscr{D}\left(H_{0}\right)$ for $k \in \mathbb{N} \cup\{0\}$, it follows that

$$
\begin{aligned}
e^{-2 k \beta i t}\left(H_{0} f_{k} \mid f_{n}\right) f_{n} & =\left(f_{n} \otimes \overline{f_{n}}\right) H_{0} \Omega_{i e^{-2 n \beta_{3}}}^{-2 i t} f_{k} \\
& =\left(f_{n} \otimes \overline{f_{n}}\right) \Omega_{l e}^{-2 i t} H_{0} H_{0} f_{k} \\
& =e^{-2 n \beta i t}\left(H_{0} f_{k} \mid f_{n}\right) f_{n},
\end{aligned}
$$

which implies that

$$
H_{0} f_{n}=\left(H_{0} f_{n} \mid f_{n}\right) f_{n}, \quad n=0,1,2, \ldots
$$


Hence we have

$$
\left\{\alpha_{n}\right\} \equiv\left\{e^{-n \beta}\left(H_{0} f_{n} \mid f_{n}\right)^{1 / 2}\right\} \in \mathbb{S}_{+} \text {and } \phi_{\rho}=\phi_{\left[\alpha_{n}\right]^{\bullet}}
$$

Suppose $\phi_{\rho}$ is $\phi_{\left\{e^{-n \beta}\right\}}$-dominated. Then $\phi$ is represented as

$$
\phi_{\rho}(x)=\left\langle\pi_{1}(x) \pi^{\prime}\left(H_{0}\right) \Omega_{\left\{e^{-n \beta_{\}}}\right.} \mid \pi^{\prime}\left(H_{0}\right) \Omega_{\left\{e^{-n \beta_{1}}\right.}\right\rangle, \quad x \in \mathscr{A}
$$

for some positive operator $H_{0}$ in $\mathscr{B}\left(L^{2}\right)$, and hence we can take $\pi^{\prime}\left(H_{0}\right) \Omega_{\left\{e^{-n \beta_{1}}\right.} \in \mathscr{S} \otimes \overline{L^{2}}$ as $\rho$. Since

$$
\begin{aligned}
& \Omega_{\left[e^{-n \beta_{3}}\right.}^{-1} \pi^{\prime}\left(H_{0}\right) \Omega_{e^{-n \beta_{3}}}=H_{0} \in \mathscr{B}\left(L^{2}\right), \\
& \overline{\left(\pi^{\prime}\left(H_{0}\right) \Omega_{\left\{e^{-n \beta_{\}}}\right.}\right)^{2} \Omega_{\left[e^{-n \beta_{3}}\right.}^{-1}}=\left(\pi^{\prime}\left(H_{0}\right) \Omega_{\left\{e^{-n \beta}\right\}}\right) H_{0} \in \mathscr{S} \otimes \bar{L}^{2},
\end{aligned}
$$

it follows from the above fact that $\phi_{\rho}=\phi_{\left\{\alpha_{n}\right\}}$ for some $\left\{\alpha_{n}\right\} \in \mathbb{S}_{+}$。

(3) A positive linear functional $\phi$ on $\mathscr{A}$ which satisfies the KMScondition with respect to $\left\{\Delta_{\left[e^{-n \beta}\right\}}^{i t}\right\}_{t \in \mathbf{R}}$ is represented as

$$
\phi=\gamma \phi_{\left(e^{-n \beta_{3}}\right)}
$$

for some constant $\gamma>0$ ([10] Theorem 30).

Example 6. 3. $_{0}$ We consider $\phi_{\left\{e^{-n \beta_{\}}}\right.}$-absolute continuity, $\phi_{\left(e^{-n \beta_{3}}\right.}-$ singularity and $\left\{\sigma_{t}^{\Omega}\left\{e^{-n \beta}\right\}\right\}_{t \in \mathbb{R}}$-invariance of positive linear functionals on $\mathscr{L}^{\dagger}(\mathscr{S})$. The following examples $(1) \sim(4)$ are modifications of examples constructed by Kosaki in [19].

(1) $\phi_{f_{\infty} \otimes \overline{f_{\infty}}}$ is $\phi_{\left\{e^{-n \beta}\right\}}-$ singular, where $f_{\infty}=\sum_{n=0}^{\infty} e^{-n \beta} f_{n} \in \mathscr{S}$.

In fact, for each $X \in \mathscr{L}^{\dagger}(\mathscr{S})$ we put

$$
X_{m}=\frac{1}{\log m} \sum_{k=1}^{m} \frac{1}{k} e^{\beta k}\left(X f_{\infty} \otimes \overline{f_{k}}\right), \quad m=2,3, \ldots
$$

Then we have

$$
\begin{aligned}
& X_{m} \in \mathscr{S} \otimes \overline{L^{2}}, \\
& \pi\left(X_{m}\right) \Omega_{\left\{e^{-n \beta_{\}}}\right.}=\frac{1}{\log m} \sum_{k=1}^{m} \frac{1}{k}\left(X f_{\infty} \mid \overline{f_{k}}\right)
\end{aligned}
$$

and

$$
\pi\left(X_{m}\right)\left(f_{\infty} \otimes \overline{f_{\infty}}\right)=\left(\frac{1}{\log m} \sum_{k=1}^{m} \frac{1}{k}\right) \pi(X)\left(f_{\infty} \otimes \overline{f_{\infty}}\right)
$$

for $m=2,3, \ldots$ It hence follows that

$$
\lim _{m \rightarrow \infty} \pi\left(X_{m}\right) \Omega_{\left\{e^{-n \beta_{\}}}\right.}=0 \text { and } \lim _{m \rightarrow \infty} \pi\left(X_{m}\right)\left(f_{\infty} \otimes \overline{f_{\infty}}\right)=\pi(X)\left(f_{\infty} \otimes \overline{f_{\infty}}\right)
$$


for each $X \in \mathscr{L}^{\uparrow}(\mathscr{S})$, which means that $\phi_{f_{\infty} \otimes f_{\infty}}$ is $\phi_{\left[e^{-n \beta}\right]}$-singular.

(2) $\phi_{f_{\infty}^{\prime} \otimes \overline{f_{\infty}^{\prime}}}$ is $\phi_{\left[e^{-n \beta_{3}}\right.}-$ singular and $\phi_{f_{\infty} \otimes \overline{f_{\infty}}}+\phi_{f_{\infty}^{\prime} \otimes \overline{f_{\infty}^{\prime}}}$ is not $\phi_{\left[e^{-n \beta_{1}}\right.}-$ singular, where $f_{\infty}^{\prime}=2 f_{0}-f_{\infty}$.

In fact, it is shown in similar to (1) that $\phi_{f_{\infty}^{\prime} \otimes \overline{f_{\infty}^{\prime}}}$ is $\phi_{\left(e^{-n \beta}\right)}$-singular. We show that $\phi_{f_{\infty} \otimes \overline{f_{\infty}}}+\phi_{f_{\infty}^{\prime} \otimes \overline{f_{\infty}^{\prime}}}$ is not $\phi_{\left(e^{-n \beta_{1}}\right.}$-singular. Since

$$
\begin{aligned}
& \left(f_{\infty} \otimes \overline{f_{\infty}}\right)^{2}+\left(f_{\infty}^{\prime} \otimes \overline{f_{\infty}^{\prime}}\right)^{2}=\frac{e^{2 \beta}}{e^{2 \beta}-1}\left(f_{\infty} \otimes \overline{f_{\infty}}+f_{\infty}^{\prime} \otimes \overline{f_{\infty}^{\prime}}\right), \\
& \left(\left(f_{\infty} \otimes \overline{f_{\infty}}\right)^{2}+\left(f_{\infty}^{\prime} \otimes \overline{f_{\infty}^{\prime}}\right)^{2}\right)\left(f_{\infty}+f_{\infty}^{\prime}\right)=\frac{2 e^{2 \beta}}{e^{2 \beta}-1}\left(f_{\infty}+f_{\infty}^{\prime}\right), \\
& \left(\left(f_{\infty} \otimes \overline{f_{\infty}}\right)^{2}+\left(f_{\infty}^{\prime} \otimes \overline{f_{\infty}^{\prime}}\right)^{2}\right)\left(f_{\infty}-f_{\infty}^{\prime}\right)=\frac{2 e^{2 \beta}}{\left(e^{2 \beta}-1\right)^{2}}\left(f_{\infty}-f_{\infty}^{\prime}\right),
\end{aligned}
$$

it follows that $f_{\infty}+f_{\infty}^{\prime}=2 f_{0}$ and $f_{\infty}-f_{\infty}^{\prime}$ are eigenvectors for $\left(\left(f_{\infty} \otimes \bar{f}_{\infty}\right)^{2}\right.$ $+\left(f_{\infty}^{\prime} \otimes{\overline{f_{\infty}^{\prime}}}^{2}\right)$ with eigenvalues $\frac{2 e^{2 \beta}}{e^{2 \beta}-1}$ and $\frac{2 e^{2 \beta}}{\left(e^{2 \beta}-1\right)^{2}}$, respectively, which implies

$$
\left(f_{\infty} \otimes \overline{f_{\infty}}\right)^{2}+\left(f_{\infty}^{\prime} \otimes \overline{f_{\infty}^{\prime}}\right)^{2} \geqq \frac{2 e^{2 \beta}}{e^{2 \beta}-1}\left(f_{0} \otimes \overline{f_{0}}\right) .
$$

Hence we have

$$
\begin{aligned}
\left(\phi_{f_{\infty} \otimes \overline{f_{\infty}}}+\phi_{f_{\infty}^{\prime} \otimes \overline{f_{\infty}^{\prime}}}\right)\left(X^{\dagger} X\right) & =\operatorname{Tr}\left(\left(f_{\infty} \otimes \overline{f_{\infty}}\right)^{2}+\left(f_{\infty}^{\prime} \otimes \overline{f_{\infty}^{\prime}}\right)^{2}\right) X^{\dagger} X \\
& \geqq \frac{2 e^{2 \beta}}{e^{2 \beta}-1} \operatorname{Tr}\left(f_{0} \otimes \overline{f_{0}}\right) X^{\dagger} X \\
& =\frac{2 e^{2 \beta}}{e^{2 \beta}-1} \phi_{f_{0} \otimes \overline{f_{0}}}\left(X^{\dagger} X\right)
\end{aligned}
$$

for all $X \in \mathscr{L}^{\dagger}(\mathscr{S})$, and hence

$$
\begin{aligned}
& \phi_{f_{0} \otimes \overline{f_{0}}} \neq 0 \\
& \quad \in P\left(\mathscr{L}^{\dagger}(\mathscr{S}), \frac{2 e^{2 \beta}}{e^{2 \beta}-1}\left(\phi_{f_{\infty} \otimes \overline{f_{\infty}}}+\phi_{f_{\infty}^{\prime} \otimes \overline{f_{\infty}^{\prime}}}\right) \cap P\left(\mathscr{L}^{\dagger}(\mathscr{S}), \phi_{\left(e^{-n \beta_{1}}\right.}\right) .\right.
\end{aligned}
$$

It hence follows from Theorem 5. 2, (2) that $\left(\phi_{f_{\infty} \otimes \overline{f_{\infty}}}+\phi_{f_{\infty}^{\prime} \otimes \overline{f_{\infty}^{\prime}}}\right)$ is not $\phi_{\left(e^{-n \beta_{1}}\right.}$-singular.

(3) The strongly $\phi_{\left(e^{-n \beta_{3}}\right.}$-absolutely continuous positive linear functional $\phi_{\left(e^{-n / 2 \beta_{\}}}\right.}$on $\mathscr{L}^{\dagger}(\mathscr{S})$ dominates a positive linear functional $\phi$ on $\mathscr{L}^{\dagger}(\mathscr{S})$ which is not strongly $\phi_{\left(e^{-n \beta_{1}}\right.}-$ absolutely continuous.

Let $\mathscr{S}_{1}$ be the closed subspace of $L^{2}$ generated by $\left\{f_{1}, f_{3}, \ldots\right.$, $\left.f_{2 n+1}, \ldots\right\}$ and $P$ be the projection of $L^{2}$ onto $\mathscr{S}_{1}$. Since $\Omega_{\left(e^{-n / 2 \beta_{1}}\right.} P=$ $P \Omega_{{ }^{-n} e^{-n / 2 \beta_{\}}}}$is a non-singular compact operator on $\mathscr{S}_{1}$, it follows from 
([19] Lemma 8.8) that there exists a unitary operator $\tilde{U}$ on $\mathfrak{S}_{1}$ such that

$$
\mathscr{R}\left(\Omega_{\left\{e^{-n / 2 \beta_{\}}}\right.} P\right) \cap \tilde{U} \mathscr{R}\left(\Omega_{\left[e^{-n / 2 \beta_{\}}}\right.} P\right)=\{0\} .
$$

We here put

$$
\begin{aligned}
& \rho=\Omega_{\left\{e^{-n / 2 \beta}\right\}} U \Omega_{\left\{e^{-n / 2 \beta}\right\}}, \text { where } U=\tilde{U} P+(1-P), \\
& \phi(X)=\operatorname{Tr} \rho \rho^{*} X, \quad X \in \mathscr{L}^{\dagger}(\mathscr{S})
\end{aligned}
$$

Since

$$
\begin{aligned}
\psi\left(X^{\dagger} X\right) & =\left\|\pi(X) \Omega_{\left(e^{-n / 2 \beta_{1}}\right)} U \Omega_{\left\{e^{-n / 2 \beta_{\}}}\right.}\right\|_{2}^{2} \\
& =\| \pi^{\prime}\left(U \Omega_{\left(e^{-n / 2 \beta_{\}}}\right) \pi(X) \Omega_{\left\{e^{-n / 2 \beta_{\}}}\right.} \|_{2}^{2}}\right. \\
& \leqq\left\|\pi^{\prime}\left(U \Omega_{\left(e^{-n / 2 \beta_{1}}\right)}\right)\right\|^{2}\left\|\pi(X) \Omega_{\left\{e^{-n / 2 \beta_{\}}}\right.}\right\|_{2}^{2} \\
& \leqq \phi_{\left(e^{-n / 2 \beta_{1}}\right.}\left(X^{\dagger} X\right)
\end{aligned}
$$

for all $X \in \mathscr{L}^{\phi}(\mathscr{S})$, it follows that $\phi$ is $\phi_{\left(e^{-n / 2 \beta_{1}}\right.}$-dominated. Suppose $\phi$ is strongly $\phi_{\left[e^{-n \beta_{3}}\right.}$-absolutely continuous. By Theorem 5.2, $\phi$ is represented as

$$
\phi(X)=\left\langle\pi(X) H_{\psi}^{\prime} Q_{\left\{e^{-n \beta_{\}}}\right.} \mid H_{\psi^{\prime}}^{\prime} \Omega_{\left(e^{-n \beta_{\}}}\right.}\right\rangle, \quad X \in \mathscr{L}^{\dagger}(\mathscr{S}) 。
$$

Hence, a positive linear functional $\phi^{\prime \prime}$ on $\mathscr{B}\left(L^{2}\right)$ defined by

$$
\phi^{\prime \prime}(A)=\left\langle\pi^{\prime \prime}(A) H_{\psi^{\prime} Q^{-n \beta_{l}}} \mid H_{\psi^{\prime} Q^{-n \beta_{l}}}^{\prime}\right\rangle
$$

is faithful and strongly $\phi_{\left(e^{-n \beta}\right)}^{\prime \prime}-$ absolutely continuous, and so by ([19] Corollary 7.3) $\pi^{\prime}\left(\mathscr{B}\left(L^{2}\right)\right) \Omega_{\left(e^{-n \beta}\right\}} \cap \pi^{\prime}\left(\mathscr{B}\left(L^{2}\right)\right) \rho$ is dense in $L^{2} \otimes \overline{L^{2}}$ 。 Take an arbitrary $H \in \pi^{\prime}\left(\mathscr{B}\left(L^{2}\right)\right) \Omega_{\left\{e^{-n \beta}\right\}} \cap \pi^{\prime}\left(\mathscr{B}\left(L^{2}\right)\right) \rho$. Then, since

$$
H=\pi^{\prime}(A) \rho=\pi^{\prime}(B) \Omega_{\left.i e^{-n \beta}\right\}}, \quad A, B \in \mathscr{B}\left(L^{2}\right),
$$

we have

$$
U \Omega_{\left[e^{-n / 2 \beta}\right\}} A \xi=\Omega_{{ }^{-n} e^{\prime 2} / \beta_{\}}} B \xi
$$

for each $\xi \in L^{2}$, which implies

$$
P \Omega_{\left\{e^{-n / 2 \beta_{\}}}\right.} B \xi=\tilde{U} P \Omega_{\left\{e^{-n / 2 \beta_{\}}}\right.} A \xi \in \mathscr{R}\left(P \Omega_{\left(e^{-n / 2 \beta_{\}}}\right.}\right) \cap \tilde{U} \mathscr{R}\left(P \Omega_{\left(e^{-n / 2 \beta_{\}}}\right.}\right)=\{0\} .
$$

Hence, we have

$$
P H \xi=P \Omega_{\left(e^{-n \beta_{3}}\right.} B \xi=\Omega_{\left\{e^{-n / 2 \beta_{\}}}\right.} P \Omega_{{ }^{-e^{-n / 2 \beta_{\}}}}} B \xi=0
$$

for each $\xi \in L^{2}$, and so $\mathscr{R}(H) \subset(1-P) \mathscr{K}$, which contradicts $\pi^{\prime}\left(\mathscr{B}\left(L^{2}\right)\right) \Omega_{\left(e^{-n \beta_{3}}\right.} \cap \pi^{\prime}\left(\mathscr{B}\left(L^{2}\right)\right) \rho$ is dense in $L^{2} \otimes \overline{L^{2}}$. Hence, $\phi$ is not strongly $\phi_{\left(e^{-n \beta_{3}}\right.}$-absolutely continuous. 
(4) The Lebesgue decomposition of $\phi_{\left\{e^{-n / 2 \beta}\right\}}$ is not unique.

In fact, the strongly $\phi_{\left(e^{-n \beta}\right\}}$-absolutely continuous positive linear functional $\phi_{\left\{e^{-n / 2 \beta}\right\}}$ on $\mathscr{L}^{\dagger}(\mathscr{S})$ is decomposed into

$$
\begin{aligned}
\phi_{t e^{-n / 2 \beta_{3}}} & =\phi_{t^{-n / 2 \beta_{\}}}}+0 \\
& =\left\{\left(\phi_{\left\{e^{-n / 2 \beta_{\}}}\right.}-\psi\right)+\phi_{c}\right\}+\phi_{s}
\end{aligned}
$$

where $\phi$ is in (3). Since $\phi_{\left\{e^{-n / 2 \beta_{\}}}\right.}-\phi \leqq \phi_{\left\{e^{-n \beta_{\}}}\right.}$and $\phi_{s} \neq 0$, it follows that $\left(\left(\phi_{\left\{e^{-n / 2 \beta_{\}}}\right.}-\psi\right)+\phi_{c}\right)$ is $\phi_{\left\{e^{-n / 2 \beta_{\}}}\right.}$-dominated and strongly $\phi_{\left\{e^{-n \beta_{\}}}\right.}$-absolutely continuous and $\phi_{s} \neq 0$ is $\phi_{\left\{e^{-n \beta_{3}}\right.}$-singular, which shows that the Lebesgue decomposition of $\phi_{\left\{e^{-n / 2 \beta_{\}}}\right.}$is not unique.

(5) Every strongly $\phi_{l^{-n \beta_{\}}}}$-absolutely continuous and $\left\{{\sigma_{t}{ }^{\Omega} e^{-n \beta_{\}}}}_{\}_{t \in \mathbf{R}}-i n v a r-}\right.$ iant, strongly positive linear functional $\phi$ on $\mathscr{L}^{\dagger}(\mathscr{S})$ is represented as

$$
\phi=\phi_{\left\{\alpha_{n}\right\}}
$$

for some $\left\{\alpha_{n}\right\} \in s_{+}$.

In fact, by Theorem $5.6 \phi$ is represented as

$$
\phi(X)=\left\langle\pi(X) H \Omega_{\left\{e^{-n \beta_{\}}}\right.} \mid H \Omega_{\left\{e^{-n \beta_{\gamma}}\right\}}\right\rangle, \quad X \in \mathscr{L}^{\dagger}(\mathscr{S})
$$

for some positive self-adjoint operator $H$ in $L^{2} \otimes \overline{L^{2}}$ affiliated with

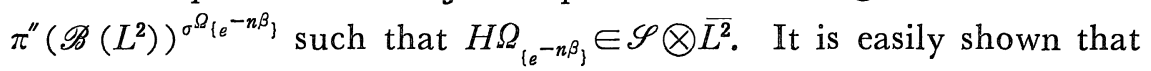

$$
\pi^{\prime \prime}\left(\mathscr{B}\left(L^{2}\right)\right)^{\sigma^{\Omega}\left(e^{-n \beta_{1}}\right.}=\left\{\pi^{\prime \prime}(A) ; A=\sum_{n=0}^{\infty} \alpha_{n} f_{n} \otimes \overline{f_{n}} \in \mathscr{B}\left(L^{2}\right)\right\} .
$$

Hence, we have

$$
H_{n}=\sum_{k=0}^{\infty} \beta_{k}^{(n)} f_{k} \otimes \overline{f_{k}} \in \mathscr{B}\left(L^{2}\right), \quad n \in \mathbb{N}
$$

and

$$
\lim _{n \rightarrow \infty} \pi^{\prime \prime}\left(H_{n}\right) \Omega_{\left\{e^{-n \beta}\right\}}=H \Omega_{\left\{e^{-n \beta}\right\}},
$$

which implies

$$
\lim _{n \rightarrow \infty} \beta_{k}^{(n)} e^{-k \beta}=\alpha_{k}, \quad k=0,1,2, \ldots
$$

and

$$
H \Omega_{\left\{e^{-n \beta_{\}}}\right.}=\sum_{k=0}^{\infty} \alpha_{k} f_{k} \otimes \overline{f_{k}} \in \mathscr{S} \otimes \overline{L^{2}},
$$

and hence $\left\{\alpha_{k}\right\} \in s_{+}$and $\phi=\phi_{\left\{\alpha_{n}\right]}$.

(6) Every strongly positive linear functional $\phi$ on $\mathscr{L}^{\dagger}(\mathscr{S})$ which 
satisfies the KMS-condition with respect to $\left\{\sigma_{t}^{\Omega\left(e^{-n \beta}\right\}}\right\}_{t \in \mathbb{R}}$ is represented as

$$
\phi=\gamma \phi_{\left\{e^{-n \beta_{1}}\right.}
$$

for some constant $\gamma>0$.

In fact, by Theorem $5.5 \phi$ is represented as

$$
\phi(X)=\left\langle\pi(X) H \Omega_{\left\{e^{-n \beta_{\}}}\right.} \mid H \Omega_{\left\{e^{-n \beta_{\}}}\right.}\right\rangle, \quad X \in \mathscr{L}^{\dagger}(\mathscr{S})
$$

for some positive self-adjoint operator $H$ affiliated with $\pi^{\prime \prime}\left(\mathscr{B}\left(L^{2}\right)\right)$ $\cap \pi^{\prime}\left(\mathscr{B}\left(L^{2}\right)\right)$ such that $H \Omega_{\left\{e^{-n \beta}\right\}} \in \mathscr{S} \otimes \overline{L^{2}}$ 。 It is easily shown that $\pi^{\prime \prime}\left(\mathscr{B}\left(L^{2}\right)\right) \cap \pi^{\prime}\left(\mathscr{B}\left(L^{2}\right)\right)=\mathbb{C} I$, which implies $\phi=\gamma \phi_{\left\{e^{-n \beta}\right\}}$ for some constant $\gamma>0$.

\section{References}

[1] Araki, H., Some properties of modular conjugation operator of von Neumann algebras and a non-commutative Radon-Nikodym theorem with a chain rule, Pacific J.Math., 50 (1974), 309-354.

[2] Araki, H. and Masuda, T., Positive cones and $L_{p}$-spaces for von Neumann algebras, Publ. RIMS, Kyoto Univ., 18 (1982), 339-411.

[3] Connes, A., Une classification de facteurs de type III, Ann. École Norm. Sup., 4-ieme Sér., 6 (1973), 133-252.

[4] Caractérisation das espaces vectoriels ordonnés sous-jacents aux algebres de von Neumann, Ann. Inst. Fourier, Grenoble., 24 (1974), 121-155.

[5] Diximier, J., Les algèbres d'opérateurs dans l'espace Hilbertian, Gausthier-Villars, Paris, 2é edition, 1969.

[6] Dye, H., A Radon-Nikodym theorem for finite rings of operators, Trans. Amer. Math. Soc., 72 (1952), 243-280.

[7] Epifanio, G. and Trapani, C., $V^{*}$-algebras: an extension of the concept of von Neumann aglebras to unbounded operators, J. Math. Phys., 25 (1984), 2633-2637.

[8] Gudder, S.P., A Radon-Nikodym theorem for *-algebras, Pacific J. Math., 70 (1977), 369-382.

[9] Gudder, S. P. and Scruggs, W., Unbounded representations of *-algebras, Pacific J. Math., 70 (1977), 369-382.

[10] Gudder, S. P. and Hudson, R. L., A noncommutative probability theory, Trans. Amer. Math. Soc., 245 (1978), 1-41.

[11] Haagerup, U., The standard form of von Neumann algebras, Math. Scand., 37 (1975), 271-283.

[12] Inoue, A., Unbounded generalization of left Hilbert algebras I, II, J. Functional Analysis, 34 (1979), 339-362: J. Functional Analysis, 35 (1980), 230-250.

[13] - A Radon-Nikodym theorem for positive linear functionals on *-algebras, $J$. Operator Theory, 10 (1983), 77-86.

[14] - An unbounded generalization of the Tomita-Takesaki theory, Publ. RIMS, Kyoto Univ., 22 (1986), 725-765.

[15] Inoue, A. and Takesue, K., Self-adjoint representations of polynomial algebras, Trans. Amer. Math. Soc., 280 (1983), 393-400.

[16] Inoue, A., Ueda, H. and Yamauchi, T., Commutants and bicommutants of algebras of unbounded operators, J. Math. Phys., 28 (1987), 1-7. 
[17] Jørgensen, P., Unbounded operators: Perturbations and commutativity, J. Functional Analysis, 39 (1980), 281-307.

[18] Katavolos, K. and Koch, I., Extension of Tomita-Takesaki theory to the unbounded algebra of the canonical commutation relations, Rep. Math. Phys., 16 (1979), 335-352.

[19] Kosaki, H., Lebesgue decomposition of states on a von Neumann algebra, Amer $J$. Math., 107 (1985), 697-735.

[20] Lassner, G., Topological algebras of operators, Rep. Math. Phys., 3 (1972), 279-293.

[21] - Topological algebras and their applications in quantum statistics, Wiss. $Z$. Karl-Marx-Univ. Leipzig, Math.-Naturwiss. R. 30 (1981), 572-595.

[22] - Algebras of unbounded operators and quantum dynamics, Physica, $124 \mathrm{~A}$ (1984), 471-480.

[23] Mathot, F., Topological properties of unbounded bicommutants, J. Math. Phys., 26 (1985), 1118-1124.

[24] Pedersen, G. and Takesaki, M., The Radon-Nikodym theorem for von Neumann algebras, Acta Math., 130 (1973), 53-88.

[25] Powers, R. T., Self-adjoint algebras of unbounded operators I, II, Commun. Math. Phys., 21 (1971), 85-124: Trans. Amer. Math. Soc., 187 (1974), 261-293.

[26] - Algebras of unbounded operators, Proc. Sym. Pure Math., 38 (1982), 389 406.

[27] Rieffel M. A. and Van Daele, A., A bounded operator approach to Tomita-Takesaki theory, Pacific J. Math., 69 (1977), 187-221.

[28] Sakai, S., A Radon-Nikodym theorem in $\mathrm{W}^{*}$-algebras, Bull. Amer. Math. Soc., 71 (1965), 149-151.

[29] Schmüdgen, K., Unbounded commutants and intertwining spaces of unbounded symmetric operators and *-representations, to appear in J. Functional Analysis.

[30] Schröder, M. and Timmermann, W., Invariance of domains and automorphisms in algebras of unbounded operators, in: Proc. Int. Conf. on Operator algebras and Group Representations, Romania 1980, 134-139.

[31] Stratila, S. and Zsidó, L., Lectures on von Neumann algebras, Editura Academiei \& Abacus Press, 1979.

[32] Takesaki, M., Tomita's theory of modular Hilbert algebras and its applications, Lecture Notes in Mathematics, 128 Springer, 1970.

[33] Takesue, K., Spatial theory for algebras of unbounded operators, Rep. Math. Phys., 21 (1985), 347-355.

[34] Tomita, M., Standard forms of von Neumann algebras, The Vth functional analysis symposium of the Math. Soc. of Japan, Sendai, 1967.

[35] Van Daele, A., A new approach to the Tomita-Takesaki Theory of generalized Hilbert algebras, J. Functional Analysis, 15 (1974), 378-393.

[36] Woronowicz, S. L., The quantum moment problem I, II, Rep. Math. Phys., 1 (1970), 135-145; Rep. Math. Phys., 1 (1971), 175-183. 